\title{
Theory and phenomenology of a two-Higgs-doublet type-II seesaw model at the $\mathrm{LHC}$ run 2
}

\author{
B. Ait Ouazghour, ${ }^{1, *}$ A. Arhrib, ${ }^{2, \dagger}$ R. Benbrik, ${ }^{1,3, \ddagger}$ M. Chabab, ${ }^{1, \S}$ and L. Rahili ${ }^{1,4, \|}$ \\ ${ }^{1}$ LPHEA, Faculty of Science Semlalia, Cadi Ayyad University, P.O.B. 2390 Marrakech, Morocco \\ ${ }^{2}$ Faculty of Science and techniques, Abdelmalek Essaadi University, B.P. 416. Tanger, Morocco \\ ${ }^{3}$ MSISM Team, Faculté Polydisciplinaire de Safi, Sidi Bouzid, B.P. 4162, Safi, Morocco \\ ${ }^{4}$ EPTHE, Faculty of Science, Ibn Zohr University, B.P 8106, Agadir, Morocco
}

(Received 17 February 2019; published 27 August 2019)

\begin{abstract}
We study the most popular nonminimal Higgs extension of the Standard Model, namely the two-Higgsdoublet model, extended by a complex triplet scalar (2HDMcT). Such model with a very small vacuum expectation value, provides a solution to the massive neutrinos through the so-called type-II seesaw mechanism. We show that the 2HDMcT enlarged parameter space allow for a rich and interesting phenomenology compatible with current experimental constraints. In this paper the 2HDMcT is subject to a detailed scrutiny. Indeed, a complete set of tree level unitarity constraints on the coupling parameters of the potential is determined, and the exact tree-level boundedness from below constraints on these couplings are generated for all directions. Moreover, we also include in our study: electroweak precision test constraints, LEP and recents LHC constraints as well as some selected set of lepton flavor violation constraints. We then perform an extensive parameter scan in the $2 \mathrm{HDMcT}$ parameter space, delimited by the above derived theoretical constraints as well as by experimental limits. We find that an important triplet admixtures are still compatible with the Higgs data and investigate which observables will allow us to restrict the triplet nature most effectively in the next runs of the LHC. Finally, we emphasize new production and decay channels and their phenomenological relevance and treatment at the LHC.
\end{abstract}

DOI: 10.1103/PhysRevD.100.035031

\section{INTRODUCTION}

After the discovery of a Standard-Model-like Higgs boson at the Large Hadron Collider (LHC) in 2012 $[1,2]$, the Standard Model (SM) of particle physics has been established as the most successful theory describing the elementary particles and their interactions. Despite its success, the SM has several drawbacks that have suggested theoretical investigations as well as experimental searches of physics beyond it. As an example, the observed neutrino oscillation cannot be explained within the SM [3]. Indeed, although the SM Higgs field is responsible for the generation of the masses of all known fundamental particles, it is unable to accommodate the tiny observed neutrino

\footnotetext{
*brahim.aitouazghour@edu.uca.ac.ma †aarhrib@gmail.com

tr.benbrik@uca.ac.ma

${ }^{\S}$ Corresponding author.

mchabab@uca.ac.ma

"rahililarbi@gmail.com
}

Published by the American Physical Society under the terms of the Creative Commons Attribution 4.0 International license. Further distribution of this work must maintain attribution to the author(s) and the published article's title, journal citation, and DOI. Funded by SCOAP ${ }^{3}$. masses. Within a renormalizable theory where new heavy fields are introduced, the neutrino masses are generated via the dimension-five Weinberg operator [4], this is the socalled seesaw mechanism. Different realizations of such a mechanism can be classified into three types: type I [5-9] in which only right-handed neutrinos coupling to the Higgs field, type II [10-13] where a new scalar field in the adjoint representation of $S U(2)_{L}$ and type III [14] which involves two extra fermionic fields. In the above seesaw mechanisms heavy fields are supplemented to the SM spectrum in such a way the desired neutrino properties are reproduced once the electroweak symmetry is broken.

In the type-II seesaw model, also dubbed Higgs triplet models (HTM), [15-20], the SM Lagrangian is augmented by a $S U(2)_{L}$ scalar triplet field $\Delta$ with hypercharge $Y_{\Delta}=2$. In HTM, neutrino masses are proportional to the vacuum expectation value (vev) of the triplet field. Hence, the small values of neutrino masses is guaranteed by the smallness of the triplet vev assumed to be less than $1 \mathrm{GeV}$ and the nonconservation of lepton number which is explicitly broken by a trilinear coupling $\mu$ term in the HTM scalar potential. The latter, protected by symmetry, is naturally small, thus ensuring small neutrino masses. The model spectrum contains several scalar particles, including a pair of singly charged Higgs boson $\left(H^{ \pm}\right)$and doubly charged 
Higgs boson $\left(H^{ \pm \pm}\right)$. In addition, it also predicts a $C P$-odd neutral scalar $\left(A^{0}\right)$ as well as two $C P$ even neutral scalars, $h$ and $H$. The lightest scalar $h$ has essentially the same couplings to the fermions and vector bosons as the Higgs boson of the SM within a large region of the HTM parameter space.

By the new discovery of 125 scalar boson at the LHC, the phenomenology of two-Higgs-doublet mod (2HDM) has been investigated broadly in the literature. In the present work, due to the similarity in mass generation mechanism between type-II seesaw and the Higgs mechanism, we extend HTM and focus on the two Higgs double model extension to the type-II seesaw model, displaying phenomenological characteristics notably different from the scalar sector emerging from the HTM. In this context, we study several Higgs processes giving rise to the production times branching ratios of heavy Higgs bosons and focus on. Unlike most of the earlier studies, we consider here a framework where both type I and type II seesaw mechanisms are implemented and contribute to neutrino mass generation. We consider a high integrated luminosity of LHC collisions at a center-of-mass energy of $13 \mathrm{TeV}$.

The content of the paper is laid out as follows. In Sec. II, we derive some crucial features of the 2HDMcT, with a focus on the particle content and the scalar potential of the model, followed by discussions on the minimization conditions of the scalar potential and the scalar mass spectra. Besides, in this work we investigate the impact of two new terms introduced in the model scalar potential. Section III, is devoted to the study of the theoretical constraints on the scalar potential parameters from tree-level vacuum stability and perturbative unitarity of the scalar sector. In Sec. IV, we further impose the electroweak precision test constraints, the LEP and recents LHC constraints associated with the $125 \mathrm{GeV}$ Higgs boson and its signal strength to delimit the parameter space. Moreover, we also discuss shortly constraints from some selected lepton flavor violating observables such as $\mu \rightarrow e \gamma$ and $\mu \rightarrow e e e$. Finally, we present some phenomenological aspects in Sec. V, benchmark points in Sec. VI and summarise our findings in Sec. VII.

\section{GENERAL CONSIDERATIONS OF 2HDM WITH TRIPLET}

\section{A. The Higgs sector}

In a model with two Higgs doublets $H_{1,2}$, the two-Higgsdoublet type-II seesaw model (2HDMcT) contains an additional $S U(2)_{L}$ triplet Higgs field $\Delta$ with hypercharge $Y=2$ and lepton number $L=-2$,

$$
H_{1}=\left(\begin{array}{c}
\phi_{1}^{+} \\
\phi_{1}^{0}
\end{array}\right), \quad H_{2}=\left(\begin{array}{c}
\phi_{2}^{+} \\
\phi_{2}^{0}
\end{array}\right), \quad \Delta=\left(\begin{array}{cc}
\delta^{+} / \sqrt{2} & \delta^{++} \\
\delta^{0} & -\delta^{+} / \sqrt{2}
\end{array}\right)
$$

The most general renormalizable and gauge invariant Lagrangian of the 2HDMcT scalar sector is given by,

$\mathcal{L}=\left(D_{\mu} H\right)^{\dagger}\left(D^{\mu} H\right)+\operatorname{Tr}\left(D_{\mu} \Delta\right)^{\dagger}\left(D^{\mu} \Delta\right)-V(H, \Delta)+\mathcal{L}_{\text {Yukawa }}$

where the scalar potential $V(H, \Delta)$, symmetric under a group $S U(2)_{L} \times U(1)_{Y}$, reads as [21]

$V\left(H_{i}, \Delta\right)=V\left(H_{1}, H_{2}\right)+V(\Delta)+V_{\text {int }}\left(H_{1}, H_{2}, \Delta\right)$

where:

$$
\begin{aligned}
& V\left(H_{1}, H_{2}\right)= m_{1}^{2} H_{1}^{\dagger} H_{1}+m_{2}^{2} H_{2}^{\dagger} H_{2}-m_{3}^{2}\left(H_{1}^{\dagger} H_{2}+H_{2}^{\dagger} H_{1}\right) \\
&+ \frac{\lambda_{1}}{2}\left(H_{1}^{\dagger} H_{1}\right)^{2}+\frac{\lambda_{2}}{2}\left(H_{2}^{\dagger} H_{2}\right)^{2}+\lambda_{3} H_{1}^{\dagger} H_{1} H_{2}^{\dagger} H_{2} \\
&+ \lambda_{4} H_{1}^{\dagger} H_{2} H_{2}^{\dagger} H_{1}+\frac{\lambda_{5}}{2}\left[\left(H_{1}^{\dagger} H_{2}\right)^{2}+\left(H_{2}^{\dagger} H_{1}\right)^{2}\right] \\
& V(\Delta)=m_{\Delta}^{2} \operatorname{Tr}\left(\Delta^{\dagger} \Delta\right)+\bar{\lambda}_{8}\left(\operatorname{Tr} \Delta^{\dagger} \Delta\right)^{2}+\bar{\lambda}_{9} \operatorname{Tr}\left(\Delta^{\dagger} \Delta\right)^{2} \\
& V_{\text {int }}\left(H_{1}, H_{2}, \Delta\right)=\left[\mu_{1} H_{1}^{T} i \sigma^{2} \Delta^{\dagger} H_{1}+\mu_{2} H_{2}^{T} i \sigma^{2} \Delta^{\dagger} H_{2}\right. \\
&\left.+\mu_{3} H_{1}^{T} i \sigma^{2} \Delta^{\dagger} H_{2}+\mathrm{H}_{\mathrm{c}} .\right] \\
&+\lambda_{6} H_{1}^{\dagger} H_{1} \operatorname{Tr} \Delta^{\dagger} \Delta+\lambda_{7} H_{2}^{\dagger} H_{2} \operatorname{Tr} \Delta^{\dagger} \Delta \\
&+\lambda_{8} H_{1}^{\dagger} \Delta \Delta^{\dagger} H_{1}+\lambda_{9} H_{2}^{\dagger} \Delta \Delta^{\dagger} H_{2}
\end{aligned}
$$

In the above, $m_{i}^{2}, i=1,2,3$ and $m_{\Delta}^{2}$ are mass squared parameters, $\lambda_{i}, i=1, \ldots, 5$ are dimensionless couplings not related to the triplet, $\bar{\lambda}_{i}, i=8,9$ are dimensionless couplings related to the triplet field, while $\mu_{i}, i=1,2,3$ with $\lambda_{i}, i=6, \ldots, 9$, are dimensionless couplings that mixe all three Higgs fields. In Eq. (6), Tr denotes the trace over $2 \times 2$ matrices, where for convenience we have used the $2 \times 2$ traceless matrix representation for the triplet. Also, the potential defined in Eq. (6) exhausts all possible gauge invariant renormalizable operators. For instance, a terms of the form $\lambda_{10} H_{1}^{\dagger} \Delta^{\dagger} \Delta H_{1}$ and $\lambda_{11} H_{2}^{\dagger} \Delta^{\dagger} \Delta H_{2}$ [21], which would be legitimate to add if $\Delta$ contained a singlet component, can actually be projected on the $\lambda_{6,7}$ and $\lambda_{8,9}$ operators appearing in Eq. (6) thanks to the identity $H_{i}^{\dagger} \Delta^{\dagger} \Delta H_{i}+H_{i}^{\dagger} \Delta \Delta^{\dagger} H_{i}=H_{i}^{\dagger} H_{i} \operatorname{Tr}\left(\Delta^{\dagger} \Delta\right)$ which is valid because $\Delta$ is a traceless $2 \times 2$ matrix.

Subsequently, we will assume that all these parameters are real valued. Indeed, apart from the $\mu_{i}$ terms, all the other operators in $V\left(H_{i}, \Delta\right)$ are self-conjugate so that, by hermicity of the potential, only the real parts of the $\lambda$ 's and the $m_{1}^{2}, m_{2}^{2}, m_{\Delta}^{2}$ mass parameters are relevant. As for $\mu_{i}$, the only parameters that can pick up a would be $C P$ phases, these phases are unphysical and can always be absorbed in a redefinition of the fields $H_{i}$ and $\Delta$. One thus concludes that the 2HDMcT Lagrangian is $C P$ conserving. 
The electroweak symmetry is spontaneously broken when the neutral components of the Higgs fields acquire vacuum expectation values $v_{1}, v_{2}$, and $v_{t}$. Thus we can shift the Higgs fields in the following way,

$\phi_{1}^{0}=\frac{v_{1}+\rho_{1}+i \eta_{1}}{\sqrt{2}}, \quad \phi_{2}^{0}=\frac{v_{2}+\rho_{2}+i \eta_{2}}{\sqrt{2}}, \quad \delta^{0}=\frac{v_{t}+\rho_{0}+i \eta_{0}}{\sqrt{2}}$ finding minimization conditions, or tree-level tadpole equations, given by

$$
V_{\text {linear }}=T_{1} \rho_{1}+T_{2} \rho_{2}+T_{3} \rho_{0}=0,
$$

where it is safe to take $T_{i}=0(i=1,2,3)$ which leads to,

$$
\begin{aligned}
& m_{1}^{2}=\frac{2 m_{3}^{2} v_{2}+\sqrt{2}\left(2 \mu_{1} v_{1}+\mu_{3} v_{2}\right) v_{t}-v_{1}\left(\lambda_{1} v_{1}^{2}+\lambda_{345} v_{2}^{2}+\lambda_{68}^{+} v_{t}^{2}\right)}{2 v_{1}} \\
& m_{2}^{2}=\frac{2 m_{3}^{2} v_{1}+\sqrt{2}\left(2 \mu_{2} v_{2}+\mu_{3} v_{1}\right) v_{t}-v_{2}\left(\lambda_{2} v_{2}^{2}+\lambda_{345} v_{1}^{2}+\lambda_{79}^{+} v_{t}^{2}\right)}{2 v_{2}} \\
& m_{\Delta}^{2}=\frac{\sqrt{2}\left(\mu_{1} v_{1}^{2}+v_{2}\left(\mu_{3} v_{1}+\mu_{2} v_{2}\right)\right)-v_{t}\left(\lambda_{68}^{+} v_{1}^{2}+2 \bar{\lambda}_{89}^{+} v_{t}^{2}+\lambda_{79}^{+} v_{2}^{2}\right)}{2 v_{t}}
\end{aligned}
$$

with $\lambda_{i j}^{+}=\lambda_{i}+\lambda_{j}, \bar{\lambda}_{i j}^{+}=\bar{\lambda}_{i}+\bar{\lambda}_{j}$ and $\lambda_{345}=\lambda_{3}+\lambda_{4}+\lambda_{5}$. If the terms associated to $v_{t}^{3}$ are omitted in Eq. (11), then we can derive a new expression for $v_{t}$ as a function of the triplet scalar mass,

$$
v_{t} \approx \frac{\mu_{1} v_{1}^{2}+\mu_{3} v_{1} v_{2}+\mu_{2} v_{2}^{2}}{\sqrt{2}\left(m_{\Delta}^{2}+\lambda_{68}^{+} v_{1}^{2} / 2+\lambda_{79}^{+} v_{2}^{2} / 2\right)}
$$

Furthermore, for $m_{\Delta}$ sufficiently large compared to $v_{1,2}$, we see that the above formula reduces to, $v_{t} \sim \mu_{1} v_{1}^{2} / \sqrt{2} m_{\Delta}^{2}$, which is referred as type-II seesaw mechanism. ${ }^{1}$

The 2HDMcT model has altogether 24 degrees of freedom: 21 parameters originating from the scalar potential given by Eq. (3) and tree vacuum expectation values of the Higgs doublets and triplet fields. However, thanks to the three minimization conditions, the $W$ gauge boson mass and the correct electroweak scales, the parameters $m_{1}^{2}, m_{2}^{2}$, $m_{\Delta}^{2}$ and $v$ can be eliminated.

\section{B. Higgs masses and mixing angles}

In what follows, we will use Eqs. (9), (10), and (11) to trade the mass parameters $m_{11}^{2}, m_{22}^{2}$, and $m_{\Delta}^{2}$ for the rest of parameters given in the potential. Thus, the $14 \times 14$ squared mass matrix is given by,

$$
\mathcal{M}^{2}=\left.\frac{1}{2} \frac{\partial^{2} V}{\partial \eta_{i}^{2}}\right|_{H_{i}=\left\langle H_{i}\right\rangle, \Delta=\langle\Delta\rangle}
$$

by denoting the corresponding VEV's

\footnotetext{
${ }^{1}$ In the absence of $\mu_{1}, \mu_{2}$, and $\mu_{3}$ the $m_{\Delta}^{2}$ becomes negative leading to a spontaneous violation of lepton number. The resulting Higgs spectrum contains a massless triplet scalar, called Majoron $J$. This model was excluded by LEP.
}

$$
\begin{aligned}
\left\langle H_{1}\right\rangle & =\left(\begin{array}{c}
0 \\
v_{1} / \sqrt{2}
\end{array}\right), \quad\left\langle H_{2}\right\rangle=\left(\begin{array}{c}
0 \\
v_{2} / \sqrt{2}
\end{array}\right) \quad \text { and } \\
\langle\Delta\rangle & =\left(\begin{array}{cc}
0 & 0 \\
v_{t} / \sqrt{2} & 0
\end{array}\right),
\end{aligned}
$$

Eq. (3) can be recast in a block diagonal form of one doubly degenerate eigenvalue $m_{H^{ \pm \pm}}^{2}$ and three $3 \times 3$ matrices denoted in the following by $\mathcal{M}_{ \pm}^{2}, \mathcal{M}_{\mathcal{C} \mathcal{P}_{\text {odd }}}^{2}$, and $\mathcal{M}_{\mathcal{C} \mathcal{P}_{\text {even }}}^{2}$. The bilinear part of the Higgs potential is then given by:

$$
\begin{aligned}
V_{H_{1}, H_{2}, \Delta}^{(2)}= & \frac{1}{2}\left(\rho_{1}, \rho_{2}, \delta^{0}\right) \mathcal{M}_{\mathcal{C} \mathcal{P}_{\text {even }}}^{2}\left(\begin{array}{c}
\rho_{1} \\
\rho_{2} \\
\rho_{0}
\end{array}\right) \\
& +\frac{1}{2}\left(\eta_{1}, \eta_{2}, \eta_{0}\right) \mathcal{M}_{\mathcal{C} \mathcal{P}_{\text {odd }}}^{2}\left(\begin{array}{c}
\eta_{1} \\
\eta_{2} \\
\eta_{0}
\end{array}\right) \\
& +\left(\phi_{1}^{-}, \phi_{2}^{-}, \delta^{-}\right) \mathcal{M}_{ \pm}^{2}\left(\begin{array}{c}
\phi_{1}^{+} \\
\phi_{2}^{+} \\
\delta^{+}
\end{array}\right) \\
& +\delta^{++} \mathcal{M}_{ \pm \pm}^{2} \delta^{--} \ldots,
\end{aligned}
$$

at tree-level. The elements of these mass matrices are explicitly presented below.

\section{Mass of the doubly charged field}

The double eigenvalue $m_{H^{ \pm \pm}}^{2}$, corresponding to the doubly charged eigenstate $\delta^{ \pm \pm}$, can simply be determined by collecting all the coefficients of $\delta^{++} \delta^{--}$in the scalar potential. It is given by 


$$
m_{H^{ \pm \pm}}^{2}=\frac{\sqrt{2} \mu_{1} v_{1}^{2}+\sqrt{2} \mu_{3} v_{1} v_{2}+\sqrt{2} \mu_{2} v_{2}^{2}-\lambda_{8} v_{1}^{2} v_{t}-\lambda_{9} v_{2}^{2} v_{t}-2 \bar{\lambda}_{9} v_{t}^{3}}{2 v_{t}}
$$

\section{Mass of the simply charged field}

The mass-squared matrix for the simply charged field in the $\left(\phi_{1}^{-}, \phi_{2}^{-}, \delta^{-}\right)$basis reads as:

$$
\mathcal{M}_{ \pm}^{2}=\left(\begin{array}{ccc}
\mathcal{M}_{11}^{ \pm} & \frac{1}{2}\left(\lambda_{45}^{+} v_{1} v_{2}-2 m_{3}^{2}\right) & \frac{1}{4}\left(v_{1} A-2 \mu_{3} v_{2}\right) \\
\frac{1}{2}\left(\lambda_{45}^{+} v_{1} v_{2}-2 m_{3}^{2}\right) & \mathcal{M}_{22}^{ \pm} & \frac{1}{4}\left(v_{2} B-2 \mu_{3} v_{1}\right) \\
\frac{1}{4}\left(v_{1} A-2 \mu_{3} v_{2}\right) & \frac{1}{4}\left(v_{2} B-2 \mu_{3} v_{1}\right) & \mathcal{M}_{33}^{ \pm}
\end{array}\right)
$$

where $A=\sqrt{2} \lambda_{8} v_{t}-4 \mu_{1}, B=\sqrt{2} \lambda_{9} v_{t}-4 \mu_{2}$ and the diagonal terms are given by,

$$
\begin{aligned}
\mathcal{M}_{11}^{ \pm} & =\frac{2 m_{3}^{2} v_{2}+v_{1} v_{t}\left(2 \sqrt{2} \mu_{1}-\lambda_{8} v_{t}\right)+\sqrt{2} \mu_{3} v_{2} v_{t}-\lambda_{45}^{+} v_{1} v_{2}^{2}}{2 v_{1}} \\
\mathcal{M}_{22}^{ \pm} & =\frac{2 m_{3}^{2} v_{1}+v_{2} v_{t}\left(2 \sqrt{2} \mu_{2}-\lambda_{9} v_{t}\right)+\sqrt{2} \mu_{3} v_{1} v_{t}-\lambda_{45}^{+} v_{1}^{2} v_{2}}{2 v_{2}} \\
\mathcal{M}_{33}^{ \pm} & =\frac{v_{1}^{2}\left(2 \sqrt{2} \mu_{1}-\lambda_{8} v_{t}\right)+v_{2}^{2}\left(2 \sqrt{2} \mu_{2}-\lambda_{9} v_{t}\right)+2 \sqrt{2} \mu_{3} v_{2} v_{1}-2 \bar{\lambda}_{9} v_{t}^{3}}{4 v_{t}} .
\end{aligned}
$$

Among the three eigenvalues of this matrix, one is zero and corresponds to the charged Goldstone bosons $G^{ \pm}$, while the two others correspond to the singly charged Higgs bosons denoted by $m_{H_{1}^{ \pm}}^{2}$ and $m_{H_{2}^{ \pm}}^{2}$ given by,

$$
m_{H_{1,2}^{ \pm}}^{2}=\frac{1}{4 v_{0}^{2} v_{t}}\left[-v_{0}\left(v_{0}\left(2 \mathcal{M}_{12}^{ \pm} \operatorname{cs}_{\beta} \operatorname{se}_{\beta} v_{t}+\kappa\right)+2 \sqrt{2} v_{t}^{2}\left(\mathcal{M}_{23}^{ \pm} \operatorname{cs}_{\beta}+\mathcal{M}_{13}^{ \pm} \operatorname{se}_{\beta}\right)\right) \mp \operatorname{cs}_{\beta} \operatorname{se}_{\beta} \sqrt{\mathcal{Y}}\right]
$$

where $\mathrm{c}_{x}, \mathrm{~s}_{x}, \mathrm{cs}_{x}, \mathrm{se}_{x}$ stand for the $\cos (x), \sin (x), \csc (x), \sec (x)$, respectively, while $v_{0}=\sqrt{v_{1}^{2}+v_{2}^{2}}, v=\sqrt{v_{1}^{2}+v_{2}^{2}+2 v_{t}^{2}}$, $\kappa=\sqrt{2} v_{0}\left(\mathcal{M}_{13}^{ \pm} c_{\beta}+\mathcal{M}_{23}^{ \pm} s_{\beta}\right)$ and

$$
\mathcal{Y}=v_{0}^{2}\left(\left(v_{0}\left(\kappa c_{\beta} s_{\beta}+2 \mathcal{M}_{12}^{ \pm} v_{t}\right)+2 \sqrt{2} v_{t}^{2}\left(\mathcal{M}_{23}^{ \pm} c_{\beta}+\mathcal{M}_{13}^{ \pm} s_{\beta}\right)\right)^{2}-4 v^{2} s_{2 \beta} v_{t}\left(\kappa \mathcal{M}_{12}^{ \pm}+2 \mathcal{M}_{13}^{ \pm} \mathcal{M}_{23}^{ \pm} v_{t}\right)\right)
$$

The above symmetric squared matrix $\mathcal{M}_{ \pm}^{2}$ is diagonalized via $\mathcal{C}$ as:

$$
\mathcal{C} \mathcal{M}_{ \pm}^{2} \mathcal{C}^{T}=\operatorname{diag}\left(m_{G^{ \pm}}^{2}, m_{H_{1}^{ \pm}}^{2}, m_{H_{2}^{ \pm}}^{2}\right)
$$

where the $\mathcal{C}$ rotation matrix is described by three mixing angles $\theta_{1}^{ \pm}, \theta_{2}^{ \pm}$, and $\theta_{3}^{ \pm}$, and the corresponding expressions for the $\mathcal{C}$ elements are give in the Appendix A as a function of the parameters inputs of our model.

\section{Mass of the neutral pseudoscalar field}

As to the mass-squared matrix for the neutral $\mathcal{C} \mathcal{P}_{\text {odd }}$ pseudoscalar field in the basis $\left(\eta_{1}, \eta_{2}, \eta_{0}\right)$, it is expressed as:

$$
\mathcal{M}_{\text {odd }}^{2}=\left(\begin{array}{ccc}
\mathcal{M}_{11}^{\text {odd }} & \frac{-2 m_{3}^{2}+\sqrt{2} \mu_{3} v_{t}+2 \lambda_{5} v_{1} v_{2}}{2} & -\frac{2 \mu_{1} v_{1}+\mu_{3} v_{2}}{\sqrt{2}} \\
\frac{-2 m_{3}^{2}+\sqrt{2} \mu_{3} v_{t}+2 \lambda_{5} v_{1} v_{2}}{2} & \mathcal{M}_{22}^{\text {odd }} & -\frac{2 \mu_{2} v_{2}+\mu_{3} v_{1}}{\sqrt{2}} \\
-\frac{2 \mu_{1} v_{1}+\mu_{3} v_{2}}{\sqrt{2}} & -\frac{2 \mu_{2} v_{2}+\mu_{3} v_{1}}{\sqrt{2}} & \mathcal{M}_{33}^{\text {odd }}
\end{array}\right)
$$

the diagonal terms are given by, 


$$
\begin{aligned}
& \mathcal{M}_{11}^{\text {odd }}=\frac{2 m_{3}^{2} v_{2}+4 \sqrt{2} \mu_{1} v_{1} v_{t}+\sqrt{2} \mu_{3} v_{2} v_{t}-2 \lambda_{5} v_{1} v_{2}^{2}}{2 v_{1}} \\
& \mathcal{M}_{22}^{\text {odd }}=\frac{2 m_{3}^{2} v_{1}+4 \sqrt{2} \mu_{2} v_{2} v_{t}+\sqrt{2} \mu_{3} v_{1} v_{t}-2 \lambda_{5} v_{1}^{2} v_{2}}{2 v_{2}} \\
& \mathcal{M}_{33}^{\text {odd }}=\frac{\eta_{1} v_{1}^{2}+\mu_{3} v_{2} v_{1}+\mu_{2} v_{2}^{2}}{\sqrt{2} v_{t}}
\end{aligned}
$$

here, $\mathcal{M}_{\mathcal{C} \mathcal{P}_{\text {odd }}}^{2}$ has three eigenvalues, one is zero corresponding to the neutral Goldstone boson $G^{0}$ while the two others are the physical states $\mathcal{C} \mathcal{P}_{\text {odd }} A_{1}$ and $A_{2}$, by setting,

$$
\begin{aligned}
\varrho= & \mathcal{M}_{23}^{\text {odd }} c_{\beta}+\mathcal{M}_{13}^{\text {odd }} s_{\beta} \\
\mathrm{F}= & \mathcal{M}_{13}^{\text {odd }} c_{\beta}+\mathcal{M}_{23}^{\text {odd }} s_{\beta} \\
\mathcal{X}= & {\left[v _ { 0 } ^ { 2 } \left(\left(v_{0}^{2} \mathrm{~F} c_{\beta} s_{\beta}+2 \mathcal{M}_{12}^{\text {odd }} v_{0} v_{t}+4 \varrho v_{t}^{2}\right)^{2}\right.\right.} \\
& \left.\left.-4 s_{2 \beta} v_{t}\left(4 v_{t}^{2}+v_{0}^{2}\right)\left(2 \mathcal{M}_{13}^{\text {odd }} \mathcal{M}_{23}^{\text {od }} v_{t}+\mathcal{M}_{12}^{\text {odd }} v_{0} \mathrm{~F}\right)\right)\right]
\end{aligned}
$$

their masses read as,

$$
\begin{aligned}
m_{A_{1,2}}^{2}= & \frac{1}{4 v_{0}^{2} v_{t}}\left[-v_{0}\left(2 \operatorname{cs}_{\beta} \operatorname{se}_{\beta} v_{t}\left(\mathcal{M}_{12}^{\text {odd }} v_{0}+2 \varrho v_{t}\right)+v_{0}^{2} \mathrm{~F}\right)\right. \\
& \left.\mp \operatorname{cs}_{\beta} \operatorname{se}_{\beta} \sqrt{\mathcal{X}}\right]
\end{aligned}
$$

while the diagonalization of such matrix is done in this case by the introduction of an unitary matrix $\mathcal{O}$ described by three mixing angles $\beta_{1}, \beta_{2}$, and $\beta_{3}$ whose expressions can be found in the Appendix A as,

$$
\mathcal{O} \mathcal{M}_{\mathcal{C} \mathcal{P}_{\text {odd }}}^{2} \mathcal{O}^{T}=\operatorname{diag}\left(m_{G^{0}}^{2}, m_{A_{1}}^{2}, m_{A_{2}}^{2}\right)
$$

\section{Mass of the neutral scalar field}

In the basis $\left(\rho_{1}, \rho_{2}, \rho_{0}\right)$ the neutral scalar mass matrix reads:

$$
\mathcal{M}_{\mathcal{C} \mathcal{P}_{\text {even }}}^{2}=\left(\begin{array}{lll}
m_{\rho_{1} \rho_{1}}^{2} & m_{\rho_{2} \rho_{1}}^{2} & m_{\rho_{0} \rho_{1}}^{2} \\
m_{\rho_{1} \rho_{2}}^{2} & m_{\rho_{2} \rho_{2}}^{2} & m_{\rho_{0} \rho_{2}}^{2} \\
m_{\rho_{1} \rho_{0}}^{2} & m_{\rho_{2} \rho_{0}}^{2} & m_{\rho_{0} \rho_{0}}^{2}
\end{array}\right)
$$

Its diagonal terms are,

$$
\begin{aligned}
& m_{\rho_{1} \rho_{1}}^{2}=\lambda_{1} v_{1}^{2}+\frac{v_{2}\left(\sqrt{2} m_{3}^{2}+\mu_{3} v_{t}\right)}{\sqrt{2} v_{1}} \\
& m_{\rho_{2} \rho_{2}}^{2}=\lambda_{2} v_{2}^{2}+\frac{v_{1}\left(\sqrt{2} m_{3}^{2}+\mu_{3} v_{t}\right)}{\sqrt{2} v_{2}} \\
& m_{\rho_{0} \rho_{0}}^{2}=\frac{4\left(\bar{\lambda}_{8}+\bar{\lambda}_{9}\right) v_{t}^{3}+\sqrt{2}\left(\mu_{1} v_{1}^{2}+\mu_{3} v_{2} v_{1}+\mu_{2} v_{2}^{2}\right)}{2 v_{t}}
\end{aligned}
$$

while the off-diagonal terms are given by,

$$
\begin{aligned}
& m_{\rho_{2} \rho_{1}}^{2}=m_{\rho_{1} \rho_{2}}^{2}=\frac{1}{\sqrt{2}}\left(\sqrt{2} v_{1} v_{2} \lambda_{345}-\sqrt{2} m_{3}^{2}-\mu_{3} v_{t}\right) \\
& m_{\rho_{0} \rho_{1}}^{2}=m_{\rho_{1} \rho_{0}}^{2}=\frac{1}{\sqrt{2}}\left(\sqrt{2} v_{1} v_{t}\left(\lambda_{6}+\lambda_{8}\right)-\left(2 \mu_{1} v_{1}+\mu_{3} v_{2}\right)\right) \\
& m_{\rho_{0} \rho_{2}}^{2}=m_{\rho_{2} \rho_{0}}^{2}=\frac{1}{\sqrt{2}}\left(\sqrt{2} v_{2} v_{t}\left(\lambda_{7}+\lambda_{9}\right)-\left(2 \mu_{2} v_{2}+\mu_{3} v_{1}\right)\right)
\end{aligned}
$$

The mass matrix can be diagonalized by an orthogonal matrix $\mathcal{E}$ which we parametrize as

$$
\mathcal{E}=\left(\begin{array}{ccc}
c_{\alpha_{1}} c_{\alpha_{2}} & s_{\alpha_{1}} c_{\alpha_{2}} & s_{\alpha_{2}} \\
-\left(c_{\alpha_{1}} s_{\alpha_{2}} s_{\alpha_{3}}+s_{\alpha_{1}} c_{\alpha_{3}}\right) & c_{\alpha_{1}} c_{\alpha_{3}}-s_{\alpha_{1}} s_{\alpha_{2}} s_{\alpha_{3}} & c_{\alpha_{2}} s_{\alpha_{3}} \\
-c_{\alpha_{1}} s_{\alpha_{2}} c_{\alpha_{3}}+s_{\alpha_{1}} s_{\alpha_{3}} & -\left(c_{\alpha_{1}} s_{\alpha_{3}}+s_{\alpha_{1}} s_{\alpha_{2}} c_{\alpha_{3}}\right) & c_{\alpha_{2}} c_{\alpha_{3}}
\end{array}\right)
$$

where the mixing angles $\alpha_{1}, \alpha_{2}$, and $\alpha_{3}$ can be chosen in the range

$$
-\frac{\pi}{2} \leq \alpha_{1,2,3} \leq \frac{\pi}{2}
$$

the rotation between the two basis $\left(\rho_{1}, \rho_{2}, \rho_{0}\right)$ and $\left(h_{1}, h_{2}, h_{3}\right)$ diagonalizes the mass matrix $\mathcal{M}_{\mathcal{C} \mathcal{P}_{\text {even }}}^{2}$ as,

$$
\mathcal{E} \mathcal{M}_{\mathcal{C} \mathcal{P}_{\text {even }}}^{2} \mathcal{E}^{T}=\operatorname{diag}\left(m_{h_{1}}^{2}, m_{h_{2}}^{2}, m_{h_{3}}^{2}\right)
$$

and leads to three mass eigenstates, ordered by ascending mass as:

$$
m_{h_{1}}^{2}<m_{h_{2}}^{2}<m_{h_{3}}^{2} .
$$

One choice of input parameters implemented in 2HDMcT consistes to use the following hybrid parametrization,

$$
\begin{aligned}
\mathcal{P}_{I}= & \left\{\alpha_{1}, \alpha_{2}, \alpha_{3}, m_{h_{1}}, m_{h_{2}}, m_{h_{3}}, m_{H^{ \pm \pm}}, \lambda_{1}, \lambda_{3}, \lambda_{4}, \lambda_{6}, \lambda_{8}, \bar{\lambda}_{8},\right. \\
& \left.\bar{\lambda}_{9}, \mu_{1}, v_{t}, \tan \beta\right\}
\end{aligned}
$$

in which $\tan \beta=v_{2} / v_{1}=\tan \theta_{1}^{ \pm}=\tan \beta_{1}$.

In Appendix B, we discuss the second choice of input parameters in the physical basis 2HDMcT. Using the Eqs. (32) and (16), one can easily express the reset of Lagrangian parameters in terms of those given by (34). These are given by 


$$
\begin{aligned}
& \lambda_{2}=\frac{-\mathcal{B} c_{\beta}^{2}+\lambda_{1} v_{0}^{2} c_{\beta}^{4}+\left(\mathcal{E}_{12}^{2} m_{h_{1}}^{2}+\mathcal{E}_{22}^{2} m_{h_{2}}^{2}+\mathcal{E}_{32}^{2} m_{h_{3}}^{2}\right) s_{\beta}^{2}}{v_{0}^{2} s_{\beta}^{4}} \\
& \lambda_{5}=\frac{\left(\mathcal{B}-\lambda_{34}^{+} v_{0}^{2} s_{\beta}^{2}\right) c_{\beta}-\lambda_{1} v_{0}^{2} c_{\beta}^{3}+\left(\mathcal{E}_{11} \mathcal{E}_{12} m_{h_{1}}^{2}+\mathcal{E}_{21} \mathcal{E}_{22} m_{h_{2}}^{2}+\mathcal{E}_{31} \mathcal{E}_{32} m_{h_{3}}^{2}\right) s_{\beta}}{v_{0}^{2} c_{\beta} s_{\beta}^{2}} \\
& \lambda_{9}=\frac{-\lambda_{8} v_{0}^{2} c_{\beta}^{2}+2 \mathcal{F}-2 m_{H^{ \pm \pm}}^{2}-2\left(2 \bar{\lambda}_{8}+3 \bar{\lambda}_{9}\right) v_{t}^{2}}{v_{0}^{2} s_{\beta}^{2}} \\
& \lambda_{7}=\frac{v_{0}\left(\mathcal{A} c_{\beta}+\mathcal{M} s_{\beta}\right)+v_{t}\left(-\lambda_{6} v_{0}^{2} c_{\beta}^{2}-2 \mathcal{F}+2 m_{H^{ \pm \pm}}^{2}+2\left(2 \bar{\lambda}_{8}+3 \bar{\lambda}_{9}\right) v_{t}^{2}\right)}{v_{0}^{2} s_{\beta}^{2} v_{t}} \\
& \mu_{2}=\frac{c_{\beta}\left(2 \mathcal{A}+v_{0} c_{\beta}\left(\sqrt{2} \mu_{1}-2 \lambda_{68}^{+} v_{t}\right)\right)}{\sqrt{2} v_{0} s_{\beta}^{2}} \\
& \mu_{3}=\frac{\sqrt{2}\left(-\mathcal{A} v_{0} c_{\beta}+v_{0}^{2} c_{\beta}^{2}\left(\lambda_{68}^{+} v_{t}-\sqrt{2} \mu_{1}\right)+v_{t}\left(\mathcal{E}_{13}^{2} m_{h_{1}}^{2}+\mathcal{E}_{23}^{2} m_{h_{2}}^{2}+\mathcal{E}_{33}^{2} m_{h_{3}}^{2}-2 \bar{\lambda}_{89}^{+} v_{t}^{2}\right)\right)}{v_{0}^{2} c_{\beta} s_{\beta}} \\
& m_{3}^{2}=\frac{\mathcal{A} v_{t}+v_{0} c_{\beta}\left(\mathcal{B}-v_{t}\left(\lambda_{68}^{+} v_{t}-\sqrt{2} \mu_{1}\right)\right)-\lambda_{1} v_{0}^{3} c_{\beta}^{3}}{v_{0} s_{\beta}}
\end{aligned}
$$

where $v_{0}=\sqrt{v_{1}^{2}+v_{2}^{2}}$ and

$$
\begin{aligned}
\mathcal{A} & =\mathcal{E}_{11} \mathcal{E}_{13} m_{h_{1}}^{2}+\mathcal{E}_{21} \mathcal{E}_{23} m_{h_{2}}^{2}+\mathcal{E}_{31} \mathcal{E}_{33} m_{h_{3}}^{2} \\
\mathcal{B} & =\mathcal{E}_{11}^{2} m_{h_{1}}^{2}+\mathcal{E}_{21}^{2} m_{h_{2}}^{2}+\mathcal{E}_{31}^{2} m_{h_{3}}^{2} \\
\mathcal{F} & =\mathcal{E}_{13}^{2} m_{h_{1}}^{2}+\mathcal{E}_{23}^{2} m_{h_{2}}^{2}+\mathcal{E}_{33}^{2} m_{h_{3}}^{2} \\
\mathcal{M} & =\mathcal{E}_{12} \mathcal{E}_{13} m_{h_{1}}^{2}+\mathcal{E}_{22} \mathcal{E}_{23} m_{h_{2}}^{2}+\mathcal{E}_{32} \mathcal{E}_{33} m_{h_{3}}^{2}
\end{aligned}
$$

the remaining 10 parameters consist of the 6 charged and $\mathcal{C P}$ odd sectors mixing angles given respectively by $\theta_{i}^{ \pm}$ $(i=1,2,3)$,

$$
\begin{aligned}
& \theta_{2}^{ \pm}=\sin ^{-1}\left(\mathcal{C}_{13}\right) \\
& \theta_{1}^{ \pm}=\cos ^{-1}\left(\mathcal{C}_{11} / \cos \theta_{2}^{ \pm}\right) \\
& \theta_{3}^{ \pm}=\cos ^{-1}\left(\mathcal{C}_{33} / \cos \theta_{2}^{ \pm}\right)
\end{aligned}
$$

and $\beta_{j}(j=1,2,3)$

$$
\begin{aligned}
& \beta_{2}=\sin ^{-1}\left(\mathcal{O}_{13}\right) \\
& \beta_{1}=\cos ^{-1}\left(\mathcal{O}_{11} / \cos \beta_{2}\right) \\
& \beta_{3}=\cos ^{-1}\left(\mathcal{O}_{33} / \cos \beta_{2}\right)
\end{aligned}
$$

and 4 Higgs bosons masses, two of them correspond to the charged states $H_{1,2}^{ \pm}$masses, while the two others are matched to $\mathcal{C} \mathcal{P}_{\text {odd }}$ states $A_{1,2}$ as discussed previously.

\section{Yukawa and gauge bosons textures}

$\mathcal{L}_{\text {Yukawa }}$ contains all the Yukawa sector of the SM plus one extra Yukawa term that leads after spontaneous symmetry breaking to (Majorana) mass terms for the neutrinos $m_{i j}=2 h_{i j}\left\langle\delta^{0}\right\rangle$, without requiring right-handed neutrino states,

$$
-\mathcal{L}_{\text {Yukawa }} \supset-Y_{\nu} L^{T} C \otimes i \sigma^{2} \Delta L+\text { H.c. }
$$

where $L$ denotes $S U(2)_{L}$ doublets of left-handed leptons, $Y_{\nu}$ denotes neutrino Yukawa couplings, $C$ the charge conjugation operator and $h_{i j}(i, j=e, \mu, \tau)$ is a complex and symmetric coupling. The $\mathbb{Z}_{2}$ symmetry is imposed in order to avoid tree-level FCNCs. Furthermore, and in terms of the various $\alpha_{i}$ which appear in the expressions of $\mathcal{E}_{i j}$ matrix elements, we list in Table $\mathrm{I}$, all the $\mathcal{C} \mathcal{P}_{\text {even }} h_{i}(i=1$, $2,3)$ Yukawa couplings for both type-I and type-II in the model.

On the other hand, expanding the covariant derivative $\mathrm{D}_{\mu}$, and performing the usual transformations on the gauge and scalar fields to obtain the physical fields, one can identify the Higgs couplings $H_{i}$ to the massive gauge bosons $V=W, Z$ as given in Table II. Note that in our model, The triplet field $\Delta$ does directly couple to the SM particles, so a new contribution will be appears, and the two

TABLE I. Normalized Yukawa couplings coefficients of the neutral Higgs bosons $h_{i}$ to the up-quarks, down-quarks $(u, d)$ in 2HDMcT.

\begin{tabular}{lcccccc}
\hline \hline & $C_{U}^{h_{1}}$ & $C_{D}^{h_{1}}$ & $C_{U}^{h_{2}}$ & $C_{D}^{h_{2}}$ & $C_{U}^{h_{3}}$ & $C_{D}^{h_{3}}$ \\
\hline Type-I & $\frac{\mathcal{E}_{12}}{s_{\beta}}$ & $\frac{\mathcal{E}_{12}}{s_{\beta}}$ & $\frac{\mathcal{E}_{22}}{s_{\beta}}$ & $\frac{\mathcal{E}_{22}}{s_{\beta}}$ & $\frac{\mathcal{E}_{32}}{s_{\beta}}$ & $\frac{\mathcal{E}_{32}}{s_{\beta}}$ \\
Type-II & $\frac{\mathcal{E}_{12}}{s_{\beta}}$ & $\frac{\mathcal{E}_{11}}{c_{\beta}}$ & $\frac{\mathcal{E}_{22}}{s_{\beta}}$ & $\frac{\mathcal{E}_{21}}{c_{\beta}}$ & $\frac{\mathcal{E}_{32}}{s_{\beta}}$ & $\frac{\mathcal{E}_{31}}{c_{\beta}}$ \\
\hline \hline
\end{tabular}


TABLE II. The normalized couplings of the neutral $\mathcal{C} \mathcal{P}_{\text {even }} H_{i}$ Higgs bosons to the massive gauge bosons $V=W, Z$ in 2HDMcT.

\begin{tabular}{lcc}
\hline \hline & $C_{W}^{h_{i}}$ & $C_{Z}^{h_{i}}$ \\
\hline$h_{1}$ & $\frac{v_{1}}{v} \mathcal{E}_{11}+\frac{v_{2}}{v} \mathcal{E}_{21}+2 \frac{v_{t}}{v} \mathcal{E}_{31}$ & $\frac{v_{1}}{v} \mathcal{E}_{11}+\frac{v_{2}}{v} \mathcal{E}_{21}+4 \frac{v_{t}}{v} \mathcal{E}_{31}$ \\
$h_{2}$ & $\frac{v_{1}}{v} \mathcal{E}_{12}+\frac{v_{2}}{v} \mathcal{E}_{22}+2 \frac{v_{t}}{v} \mathcal{E}_{32}$ & $\frac{v_{1}}{v} \mathcal{E}_{12}+\frac{v_{2}}{v} \mathcal{E}_{22}+4 \frac{v_{t}}{v} \mathcal{E}_{32}$ \\
$h_{3}$ & $\frac{v_{1}}{v} \mathcal{E}_{13}+\frac{v_{2}}{v} \mathcal{E}_{23}+2 \frac{v_{t}}{v} \mathcal{E}_{33}$ & $\frac{v_{1}}{v} \mathcal{E}_{13}+\frac{v_{2}}{v} \mathcal{E}_{23}+4 \frac{v_{t}}{v} \mathcal{E}_{33}$ \\
\hline \hline
\end{tabular}

couplings $C_{V}^{h_{i}}\left(V=W^{ \pm}, Z\right)$ differs from one to another by a factor 2 associated to $v_{t}$.

\section{THEORETICAL CONSTRAINTS}

\section{A. Unitarity}

Physics beyond the Standard Model (BSM) refers to the theoretical developments needed to explain the deficiencies of the SM, and any SM extension might tend to reproduce the entirety of current phenomena. Here the question usually addressed is which theory BSM is the right one, can only be settled via experiments. In our paper, we will globally scrutinise the $2 \mathrm{HDMcT}$ model, looking for the space of parameters allowed by all the theoretical constraints as well as experimental ones. In this subsection, we apply perturbative unitarity to a complex triplet field $\Delta$ coupled to the two Higgs doublet field of $2 \mathrm{HDM}$, and as usual we consider the elastic $2 \rightarrow 2$ scattering processes for this purpose. The explicit formulas for all the eigenvalues are given by,

$$
\begin{aligned}
& a_{1}^{ \pm}=\frac{1}{2}\left(\lambda_{1}+\lambda_{2} \pm \sqrt{\left(\lambda_{1}-\lambda_{2}\right)^{2}+4 \lambda_{5}^{2}},\right. \\
& a_{2}^{ \pm}=\frac{1}{2}\left(\lambda_{1}+\lambda_{2} \pm \sqrt{\lambda_{1}^{2}-2 \lambda_{2} \lambda_{1}+\lambda_{2}^{2}+4 \lambda_{5}^{2}}\right) \\
& a_{3}^{ \pm}=\lambda_{3} \pm \lambda_{4}, \quad a_{4}^{ \pm}=\lambda_{3} \pm \lambda_{5}, \quad a_{5}^{ \pm}=\lambda_{3}+2 \lambda_{4} \pm 3 \lambda_{5}, \\
& a_{6}=\lambda_{6}, \quad a_{7}=\lambda_{7}, \quad a_{8}=2 \bar{\lambda}_{8} \\
& a_{9}=\lambda_{6}+\lambda_{8}, \quad a_{10}=\lambda_{7}+\lambda_{9}, \quad a_{11}=\lambda_{6}+\frac{3}{2} \lambda_{8}, \\
& a_{12}=\lambda_{6}-\frac{1}{2} \lambda_{8}, \quad a_{13}=\lambda_{7}+\frac{3}{2} \lambda_{9} \\
& a_{14}=\lambda_{7}-\frac{1}{2} \lambda_{9}, \quad a_{15}=2\left(\bar{\lambda}_{8}+\bar{\lambda}_{9}\right), \quad a_{16}=2 \bar{\lambda}_{8}+\bar{\lambda}_{9}
\end{aligned}
$$

in addition with three other $a_{17,18,19}$ eigenvalues originating from the cubic polynomial equation,

$$
\begin{aligned}
x^{3} & -\left(\lambda_{1}+\lambda_{2}+2 \bar{\lambda}_{8}+4 \bar{\lambda}_{9}\right) x^{2} \\
& +\left(\lambda_{1} \lambda_{2}-\lambda_{4}^{2}-\lambda_{8}^{2}+2 \lambda_{1} \bar{\lambda}_{8}+2 \lambda_{2} \bar{\lambda}_{8}-\lambda_{9}^{2}+4 \lambda_{1} \bar{\lambda}_{9}+4 \lambda_{2} \bar{\lambda}_{9}\right) x \\
& +\left(\lambda_{2} \lambda_{8}^{2}-2 \lambda_{1} \lambda_{2} \bar{\lambda}_{8}+2 \lambda_{4}^{2} \bar{\lambda}_{8}-2 \lambda_{4} \lambda_{8} \lambda_{9}+\lambda_{1} \lambda_{9}^{2}\right. \\
& \left.-4 \lambda_{1} \lambda_{2} \bar{\lambda}_{9}+4 \lambda_{4}^{2} \bar{\lambda}_{9}\right)=0
\end{aligned}
$$

for which the solutions have been extensively detailed in Appendix C, Eq. (C8). To ensure the unitarity constraints, the absolute value of the above eigenvalues must be bounded from above as [22,23],

$$
\left|a_{i}\right| \leq 0.5 \quad i=1, \ldots, 19
$$

In Appendix C, we describe in more detail the scattering matrix of all the two-body processes in the scalar sector of the 2HDMcT model.

\section{B. Boundedness from below (BFB)}

In order to derive the $\mathrm{BFB}$ constraints, we require that the vacuum is stable at tree level. Generically, this means that the scalar potential has to be bounded from below at large scalar field values in any directions of the field space. Thus, the potential is asymptotically dominated by the quartic terms,

$$
\begin{aligned}
V^{(4)} & \left(H_{1}, H_{2}, \Delta\right) \\
= & \frac{\lambda_{1}}{2}\left(H_{1}^{\dagger} H_{1}\right)^{2}+\frac{\lambda_{2}}{2}\left(H_{2}^{\dagger} H_{2}\right)^{2}+\lambda_{3} H_{1}^{\dagger} H_{1} H_{2}^{\dagger} H_{2} \\
& +\lambda_{4} H_{1}^{\dagger} H_{2} H_{2}^{\dagger} H_{1}+\frac{\lambda_{5}}{2}\left[\left(H_{1}^{\dagger} H_{2}\right)^{2}+\left(H_{2}^{\dagger} H_{1}\right)^{2}\right] \\
& +\lambda_{6} H_{1}^{\dagger} H_{1} \operatorname{Tr} \Delta^{\dagger} \Delta+\lambda_{7} H_{2}^{\dagger} H_{2} \operatorname{Tr} \Delta^{\dagger} \Delta+\lambda_{8} H_{1}^{\dagger} \Delta \Delta^{\dagger} H_{1} \\
& +\lambda_{9} H_{2}^{\dagger} \Delta \Delta^{\dagger} H_{2}+\bar{\lambda}_{8}\left(\operatorname{Tr} \Delta^{\dagger} \Delta\right)^{2}+\bar{\lambda}_{9} \operatorname{Tr}\left(\Delta^{\dagger} \Delta\right)^{2} .
\end{aligned}
$$

Therefore, to obtain in this model the full set of BFB conditions valid for all directions, it is suitable to only consider $V^{(4)}\left(H_{1}, H_{2}, \Delta\right)$ than to study the full scalar potential. As an illustration, consider for instance the case where there is no coupling between doublets $H_{i}$ and triplet $\Delta$ Higgs bosons, i.e., $\lambda_{3}=\lambda_{4}=\lambda_{5}=\lambda_{6}=\lambda_{7}=\lambda_{8}=\lambda_{9}=0$. Obviously, one can see that,

$$
\lambda_{1}>0 \& \lambda_{2}>0 \& \bar{\lambda}_{8}>0 \& \bar{\lambda}_{9}>0
$$

Given this, all the other necessary and sufficient conditions for stability listed in Appendix D can be read as,

$$
\Omega_{2 \mathrm{HDM}} \cup \Omega_{1} \cup \Omega_{2} \cup \Omega_{3} \cup \Omega_{4} \cup \Omega_{5}
$$

with

$$
\begin{aligned}
& \Omega_{2 \mathrm{HDM}} \\
& \quad=\left\{\lambda_{1}, \lambda_{2}>0, \lambda_{3}+\sqrt{\lambda_{1} \lambda_{2}}>0, \lambda_{3}+\lambda_{4}-\left|\lambda_{5}\right|+\sqrt{\lambda_{1} \lambda_{2}}>0\right\}
\end{aligned}
$$

the corresponding 2HDM BFB constraints well known in the literature, whereas the $\Omega_{i}(i=1, \ldots, 5)$ stand for the new constraints added as follows, 


$$
\begin{aligned}
& \Omega_{1}=\left\{\lambda_{6}, \lambda_{7}>0, \lambda_{6}+\lambda_{8}>0, \lambda_{7}+\lambda_{9}>0, \bar{\lambda}_{8}+\bar{\lambda}_{9}>0, \bar{\lambda}_{8}+\frac{\bar{\lambda}_{9}}{2}>0\right\} \\
& \Omega_{2}=\left\{\left(\bar{\lambda}_{9} \sqrt{2 \lambda_{1}} \geq\left|\lambda_{8}\right| \sqrt{\bar{\lambda}_{8}+\bar{\lambda}_{9}}\right) \quad \text { or }\left(2 \lambda_{6}+\lambda_{8}+\sqrt{\left(4 \lambda_{1} \bar{\lambda}_{9}-\lambda_{8}^{2}\right)\left(1+2 \frac{\bar{\lambda}_{8}}{\bar{\lambda}_{9}}\right)}\right)\right\} \\
& \Omega_{3}=\left\{\left(\bar{\lambda}_{9} \sqrt{2 \lambda_{2}} \geq\left|\lambda_{9}\right| \sqrt{\bar{\lambda}_{8}+\bar{\lambda}_{9}}\right) \quad \text { or }\left(2 \lambda_{7}+\lambda_{9}+\sqrt{\left(4 \lambda_{2} \bar{\lambda}_{9}-\lambda_{9}^{2}\right)\left(1+2 \frac{\bar{\lambda}_{8}}{\bar{\lambda}_{9}}\right)}\right)\right\} \\
& \Omega_{4}=\left\{\lambda_{6}+\sqrt{2 \lambda_{1}\left(\bar{\lambda}_{8}+\bar{\lambda}_{9}\right)}>0, \lambda_{6}+\lambda_{8}+\sqrt{2 \lambda_{1}\left(\bar{\lambda}_{8}+\bar{\lambda}_{9}\right)}>0\right\} \\
& \Omega_{5}=\left\{\lambda_{7}+\sqrt{2 \lambda_{2}\left(\bar{\lambda}_{8}+\bar{\lambda}_{9}\right)}>0, \lambda_{7}+\lambda_{9}+\sqrt{2 \lambda_{2}\left(\bar{\lambda}_{8}+\bar{\lambda}_{9}\right)}>0\right\}
\end{aligned}
$$

besides other are mentioned in Appendix D.

\section{Bounds from theoretical constraints}

In order to validate our rough analytical understanding and to further explore the impact of the unitarity and BFB constraints we use the numerical machinery. There are many possibilities what to use as input parameters. Naively using the initial Lagrangian parameters will hardly produce points which are in agreement with the Higgs measurements. Therefore, we trade hybrid parameterization. With that choice, the full set of parameters is given by Eq. (34). As first step, we show in Fig. 1, all generated points in the planes $\lambda_{6}$ vs $\lambda_{8}, \lambda_{7}$ vs $\lambda_{9}$ and $\bar{\lambda}_{8}$ vs $\bar{\lambda}_{9}$.

In the $2 \mathrm{HDMcT}$, the presence of the triplet field implies a new scalar couplings $\lambda_{6,7,8,9}$ et $\bar{\lambda}_{8,9}$ and the vacuum stability condition requires that not only $\lambda_{1,2} \geq 0$ but also $\lambda_{7,8} \geq 0$ with the conditions in Eq. (47). By varying $\lambda_{1, \ldots 5}$ in $[-8 \pi: 8 \pi]$, we show in Fig. 1, the allowed domains on $\lambda_{6,7,8,9}$ and $\bar{\lambda}_{8,9}$ plans without conflicting with the theoretical constraints. We assume that the seesaw mechanism at the TeV scale that we consider here, is a "low-energy" effective phenomenological manifestation at high energy scale. We therefore assume that the couplings remain perturbative up to GUT scale.

\section{LIMITS FROM EXPERIMENTAL CONSTRAINTS}

In this section we will review the experimental constraints subject to our model. We discuss first the electroweak precision test observables EWPT S, T, and U and present the analytical expressions for $\mathrm{S}$ and $\mathrm{T}$. Second we comment on some lepton flavor violation observables (LFV) and discuss the effect of the doubly charged Higgs boson on such observables. At the end we review the collider constraints such as LHC and LEP constraints.

\section{A. Oblique parameters}

Strong indirect probe of physics beyond SM is provided by the oblique parameters $\mathrm{S}, \mathrm{T}$, and U. More than that, the calculations of several observables check their dependences on those oblique parameters, for example, and not as a limitation, the $\rho$ parameter [24], i.e., $\rho=m_{W}^{2} / m_{Z}^{2} c_{W}^{2}$. In the $\mathrm{SM}$, this parameter is equal to 1 at tree level. In the THDMcT, the new triplet contributions to $W$ and $Z$ masses readily form Eq. (14) and the kinetic terms in Eq. (2) leads us to write,

$$
m_{Z}^{2}=\frac{g^{2}\left(v_{1}^{2}+v_{2}^{2}+4 v_{t}^{2}\right)}{4 \cos ^{2} \theta_{W}}
$$
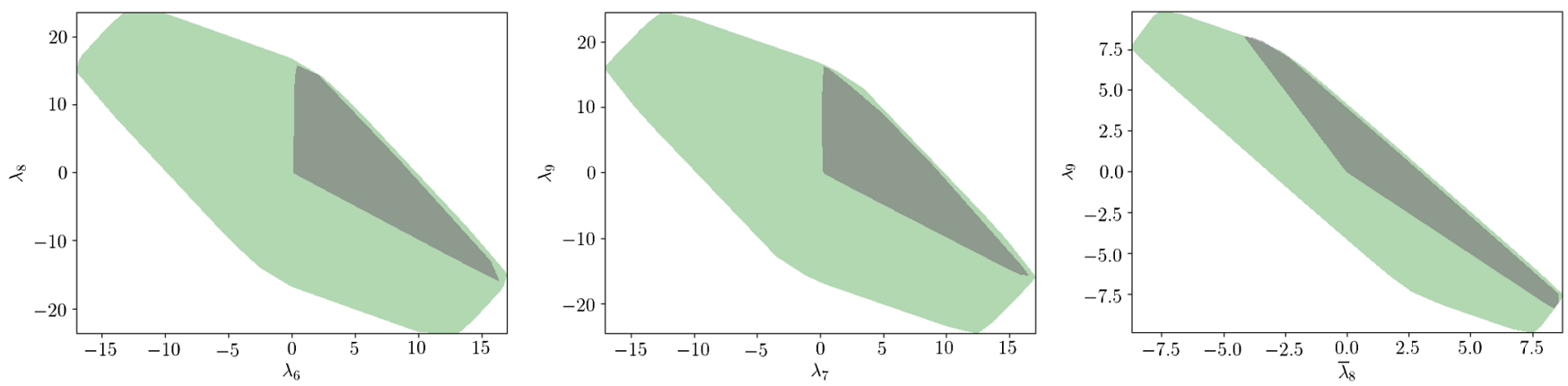

FIG. 1. The allowed ranges of $\left(\lambda_{6}, \lambda_{8}\right)$ (left), $\left(\lambda_{7}, \lambda_{9}\right)$ (middle) and $\left(\bar{\lambda}_{8}, \bar{\lambda}_{9}\right)$ (right) by imposing unitarity (green), combined unitarity and BFB (grey) constraints. 


$$
m_{W}^{2}=\frac{g^{2}\left(v_{1}^{2}+v_{2}^{2}+2 v_{t}^{2}\right)}{4}=\frac{g^{2} v^{2}}{4}
$$

the modified form of the $\rho$ parameter reads

$$
\rho=\frac{v_{0}^{2}+2 v_{t}^{2}}{v_{0}^{2}+4 v_{t}^{2}} \approx 1-2 \frac{v_{t}^{2}}{v_{0}^{2}}=1+\delta \rho \neq 0 .
$$

The impact of a 2HDMcT in the so-called electroweak precision requires that $\rho$ to be close to its SM value: $\rho=$ $1.0004_{-0.0004}^{+0.0003}$ [25]. Then, one gets an upper bound for $v_{t}<5 \mathrm{GeV}$. Furthermore, the major contribution to the T-parameter comes from the loops involving the scalar triplet when $v_{t}$ equal to zero or less. Also, since deviations from the Standard Model expectations in $U$ are negligible [26], then we will assume the latter to be zero and consider only $S$ and $T$. We compute their $\chi_{S T}^{2}$ contribution through,

$\chi_{S T}^{2}=\left(S-S^{\text {best }} T-T^{\text {best }}\right)\left(\begin{array}{ll}0.0085 & 0.0063 \\ 0.0063 & 0.0057\end{array}\right)^{-1}\left(\begin{array}{c}S-S^{\text {best }} \\ T-T^{\text {best }}\end{array}\right)$

For $U=0$, the electroweak fit gives the values.

$$
S^{\text {best }}=0.06, \quad T^{\text {best }}=0.097
$$

The contribution of the scalar triplet to $S$ and $T$ reads as [27]

$$
\begin{aligned}
\mathrm{T}= & \mathrm{T}_{2 H D M c T} \\
= & \frac{1}{4 \pi s_{w}^{2} m_{W}^{2}}\left[\sum_{+} F\left(m_{++}^{2}, m_{+}^{2}\right)+\sum_{+, 0} F\left(m_{+}^{2}, m_{0}^{2}\right)\right] \\
\mathrm{S}= & \mathrm{S}_{2 H D M c T} \\
= & -\frac{1}{3 \pi}\left[\sum_{0} \log \left(\frac{m_{++}^{2}}{m_{0}^{2}}\right)+6 \sum_{0} \xi\left(\frac{m_{0}^{2}}{m_{Z}^{2}}, \frac{m_{0}^{2}}{m_{Z}^{2}}\right)\right. \\
& \left.+6\left(1-2 s_{w}^{2}\right)^{2} \xi\left(\frac{m_{++}^{2}}{m_{Z}^{2}}, \frac{m_{++}^{2}}{m_{Z}^{2}}\right)+6 s_{w}^{4} \sum_{+} \xi\left(\frac{m_{+}^{2}}{m_{Z}^{2}}, \frac{m_{+}^{2}}{m_{Z}^{2}}\right)\right]
\end{aligned}
$$

where, $m_{++}=m_{H^{ \pm \pm}}, m_{+}=m_{H_{1}^{ \pm}}, m_{H_{2}^{ \pm}}$, and $m_{0}=m_{h_{2}}, m_{h_{3}}$, while $s_{w}$ stands for the sinus of the Weinberg angle $\theta_{w}$. The functions $\xi(x, y)$ and $F(x, y)$ are defined by,

$$
\begin{aligned}
& F(x, y)=\frac{x+y}{2}-\frac{x y}{x-y} \ln \left(\frac{x}{y}\right) \\
& \xi(x, y)=\frac{4}{9}-\frac{5}{12}(x+y)+\frac{1}{6}(x-y)^{2}+\frac{1}{4}\left[x^{2}-y^{2}-\frac{1}{3}(x-y)^{3}-\frac{x^{2}+y^{2}}{x-y}\right] \ln \frac{x}{y}-\frac{1}{12} d(x, y) f(x, y) \\
& f(x, y)= \begin{cases}-2 \sqrt{d(x, y)}\left[\arctan \frac{x-y+1}{\sqrt{d(x, y)}}-\arctan \frac{x-y-1}{\sqrt{d(x, y)}}\right] & \text { for } d(x, y)>0 \\
0 & \text { for } d(x, y)=0 \\
\sqrt{-d(x, y)} \ln \left[\frac{x+y-1+\sqrt{-d(x, y)}}{x+y-1-\sqrt{-d(x, y)}}\right] & \text { for } d(x, y)<0\end{cases} \\
& d(x, y)=-1+2(x+y)-(x-y)^{2} .
\end{aligned}
$$

\section{B. Direct LHC and LEP constraints}

The HiggsBounds code [28] is used to test a model against experimental data from LEP, Tevatron and the LHC. In our analysis we use part function of HiggsBounds version 5.2.0beta with the latest constraints for heavy Higgs bosons. The required input for HiggsBounds program are masses for all the scalar, the effective Higgs couplings, the total decay widths for all scalar and the branching ratios. The exclusion test at $2 \sigma$ is then performed on the five physical scalars of our model. HiggsBounds returns a binary result indicating if the specific model point has been excluded at $95 \%$ C.L. or not.
Both experiments ATLAS and CMS at LHC reported the discovery of a scalar particle with mass around $125.09 \mathrm{GeV}$ [29]. Meanwhile, this has been strengthen further by ATLAS and CMS with the first $13 \mathrm{TeV}$ results. In our model, we identify the Higgs field $H_{1}$ with the observed SM-like Higgs boson with a mass:

$$
m_{h_{1}}=125.09 \pm 0.4 \mathrm{GeV}
$$

We include both the measured signal rates from the ATLAS and CMS Run I and Run II and their combinations in our study via the public code HiggsSignals-2.2.1beta [30]. 
TABLE III. Best-fit parameters that enter in the neutrino mass mixing according to [3]. The neutrino masses in the IH scenario are given by $m_{2}^{2}=m_{1}^{2}+\Delta m_{21}^{2}, m_{3}^{2}=m_{2}^{2}-\Delta m_{23}^{2}$, whereas in NH case are $m_{2}^{2}=m_{1}^{2}+\Delta m_{21}^{2}, m_{3}^{2}=m_{1}^{2}+\Delta m_{31}^{2}$.

\begin{tabular}{lcc}
\hline \hline Parameter & Best-fit & Hierarachy \\
\hline$\Delta m_{21}^{2}$ & $7.37 \times 10^{-5} \mathrm{eV}^{2}$ & Any \\
$\Delta m_{31}^{2}$ & $2.56 \times 10^{-3} \mathrm{eV}^{2}$ & Normal \\
$\Delta m_{23}^{2}$ & $2.56 \times 10^{-3} \mathrm{eV}^{2}$ & Inverted \\
$\sin ^{2} \theta_{12}$ & 0.297 & Any \\
$\sin ^{2} \theta_{23}$ & Normal \\
$\sin ^{2} \theta_{23}$ & 0.425 & Inverted \\
$\sin ^{2} \theta_{13}$ & 0.589 & Normal \\
$\sin ^{2} \theta_{13}$ & 0.0215 & Inverted \\
\hline \hline
\end{tabular}

The global $\chi$-square is defined by:

$$
\chi^{2}=\chi_{H S}^{2(8 \mathrm{TeV})}+\chi_{H S}^{2(13 \mathrm{TeV})}+\chi_{S T}^{2}
$$

we then determine the minimal $\chi^{2}$ value over the scanned parameter space, $\chi_{\min }^{2}$, and keep the allowed parameter space that features a $\chi^{2}$ value within $\Delta \chi^{2}=\chi^{2}-\chi_{\min }^{2} \leq 2.3,5.99$, 11.8 (which corresponds respectively to $68 \% \mathrm{CL}, 95.5 \% \mathrm{CL}$ and $99.7 \%$ CL.)

\section{Lepton flavor violation}

One of the open problem in particle physics is lepton flavor violation and its origin. LFV if present would allow in the Lagrangian a couplings that violate lepton flavor. Therefore, these couplings would contribute to LFV decays of leptons such as: $\mu \rightarrow \bar{e} e e$ and $\mu \rightarrow e \gamma$. Note that such final states for muon decay are very clean and have been searched by various experiments. From the negative searches, several stringent experimental upper bounds have been derived.

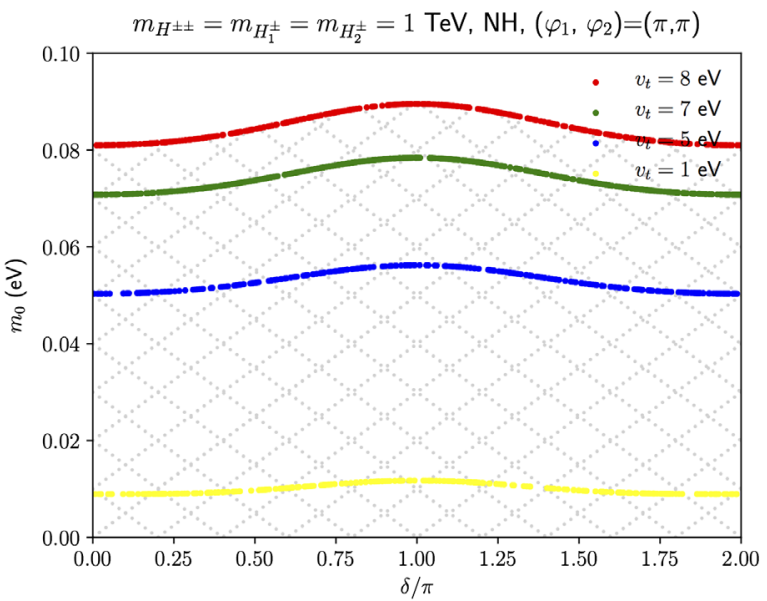

In our model 2HDMcT, lepton number violation is introduced by the Yukawa term given by Eq. (39), where $Y_{\nu}$ is a complex $3 \times 3$ matrix. After electroweak symmetry breaking one generate neutrino masses as well as lepton number violating decays of the Higgs bosons such as $H^{++} \rightarrow e_{i}^{+} e_{j}^{+}$and $H^{+} \rightarrow e_{i}^{+} \nu_{j}\left(e_{i}=e, \mu, \tau\right)$.

In the framework of $2 \mathrm{HDMcT}$, we limit ourself to the following processes: $\mu \rightarrow \bar{e} e e$ and $\mu \rightarrow e \gamma$. The first process $\mu \rightarrow \bar{e} e e$, would receive a tree level contribution from doubly charged Higgs while the loop mediated one $\mu \rightarrow e \gamma$ would receive contribution both from singly and doubly charged Higgs. Therefore, these 2 processes can set constraints on the parameter space of the neutrino mass matrix (which is proportional to $v_{t}$ ). Such processes, along with the $H^{ \pm \pm}$effect have been studied in [31-33]. The branching ratio for $\mu \rightarrow \bar{e} e e$ is given by: [34]

$$
\mathrm{BR}(\mu \rightarrow \bar{e} e e)=\frac{\left|h_{\mu e}\right|^{2}\left|h_{e e}\right|^{2}}{4 G_{F}^{2} m_{H^{ \pm \pm}}^{4}} \mathrm{BR}(\mu \rightarrow e \bar{\nu} \nu)
$$

where $\operatorname{BR}(\mu \rightarrow e \bar{\nu} \nu) \approx 100 \% . h_{i j}$ can be expressed in terms of the Pontecorvo-Maki-Nakagawa-Sakata matrix, $V_{\text {PMNS }}$ [3] and the triplet vev $v_{t}$ as follows [35]:

$$
\begin{aligned}
h_{i j} & =\frac{m_{i j}}{\sqrt{2} v_{t}} \\
& \equiv \frac{1}{\sqrt{2} v_{t}}\left[V_{\mathrm{PMNS}} \operatorname{diag}\left(m_{1}, m_{2} e^{i \varphi_{1}}, m_{3} e^{i \varphi_{2}}\right) V_{\mathrm{PMNS}}^{T}\right]_{i j}
\end{aligned}
$$

The branching ratio for $\mu \rightarrow e \gamma$ is given by: [34]

$$
\mathrm{BR}(\mu \rightarrow e \gamma)=384 \pi^{2}\left(\left|A_{R}\right|^{2}+\left|A_{L}\right|^{2}\right) .
$$

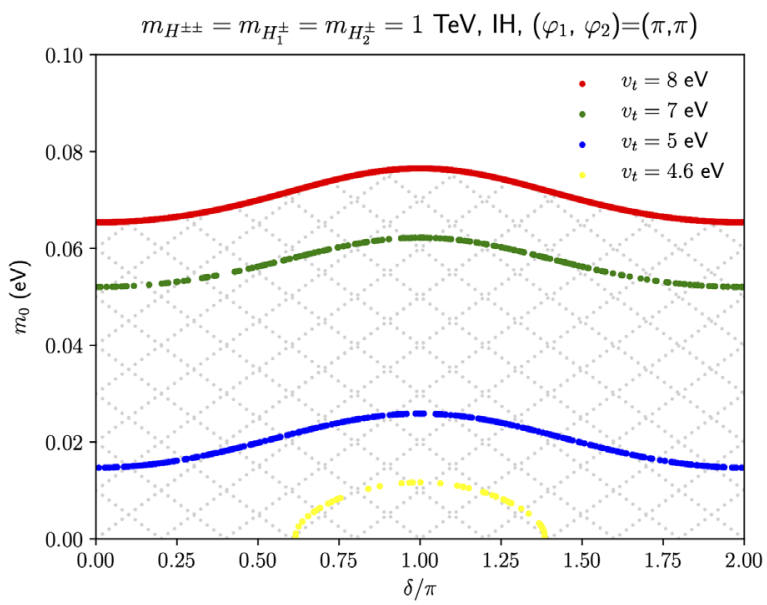

FIG. 2. The allowed area (in dashed) for various values of $v_{t}$ in the $\left(m_{1}, \delta\right)$ plane in the framework of 2HDMcT type-II. Left side stands for the $\mathrm{IH}$ and the right for $\mathrm{NH}$. 

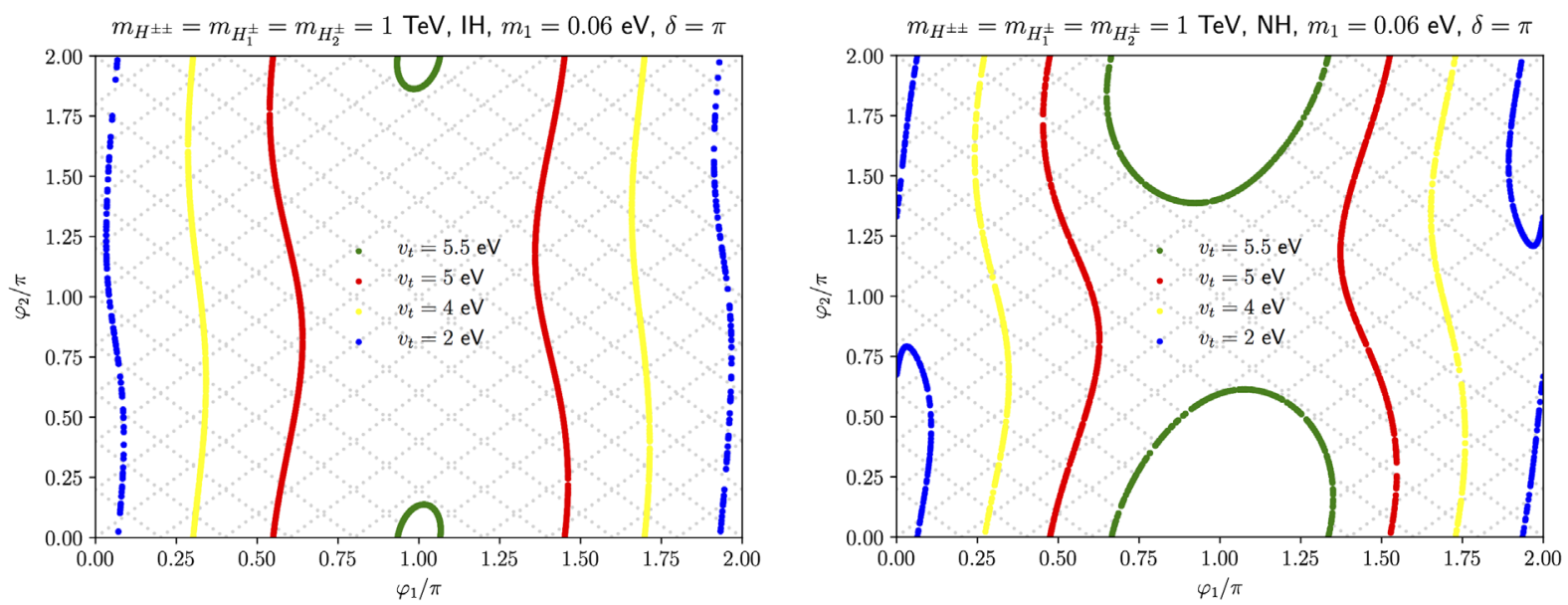

FIG. 3. The allowed area (in dashed) for various values of $v_{t}$ in the $\left(\varphi_{1}, \varphi_{2}\right)$ plane in the framework of $2 \mathrm{HDMcT}$ type-II, setting $m_{1}=0.06 \mathrm{eV}$. Left side stands for the $\mathrm{IH}$ and the right for $\mathrm{NH}$.
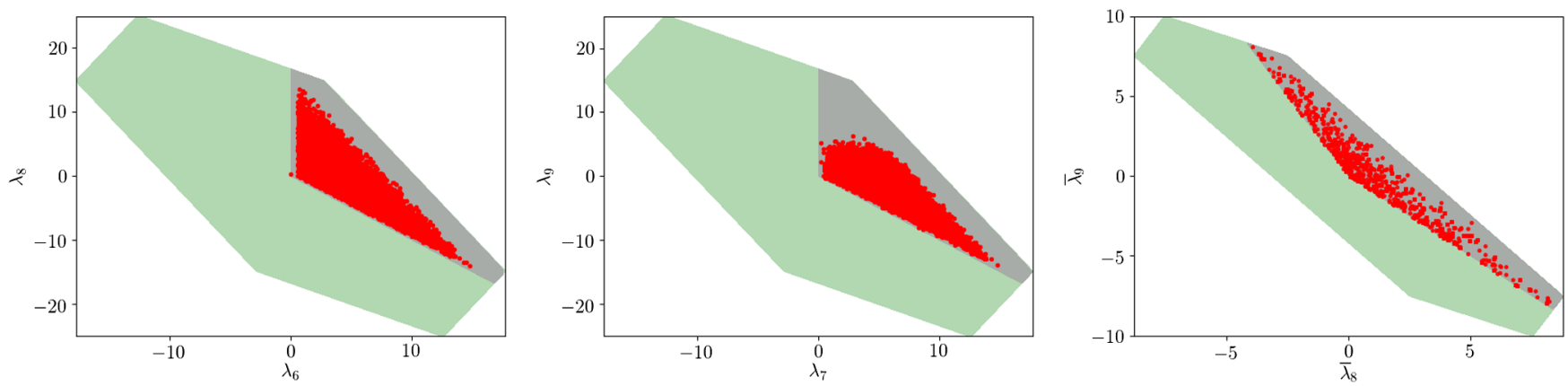

FIG. 4. The largest $\left(\lambda_{6}, \lambda_{8}\right)$ (left), $\left(\lambda_{7}, \lambda_{8}\right)$ (center) and $\left(\bar{\lambda}_{8}, \bar{\lambda}_{9}\right)$ (right) domain allowed by $C_{1}$ (gray) and $C_{2}$ (red).
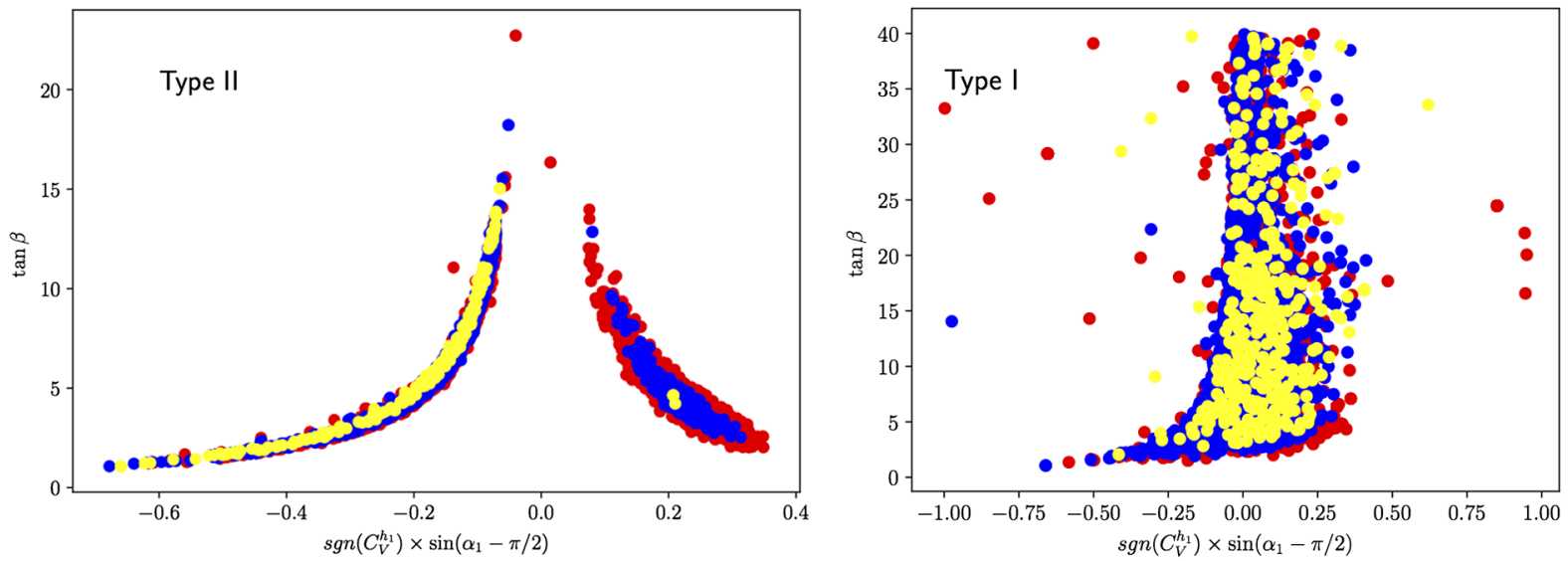

FIG. 5. The allowed regions in $\left(\operatorname{sgn}\left(C_{V}^{h_{1}}\right) \sin \left(\alpha_{1}-\pi / 2\right), \tan \beta\right)$ after imposing $C_{1}$ and $C_{2}$ constraints, where the left and right panels represent the allowed values in type-II(left), type-I(right), respectively. The errors for $\chi$-square fit are 99.7\% CL (red), 95.5\% CL (blue) and $68 \%$ CL (yellow). 

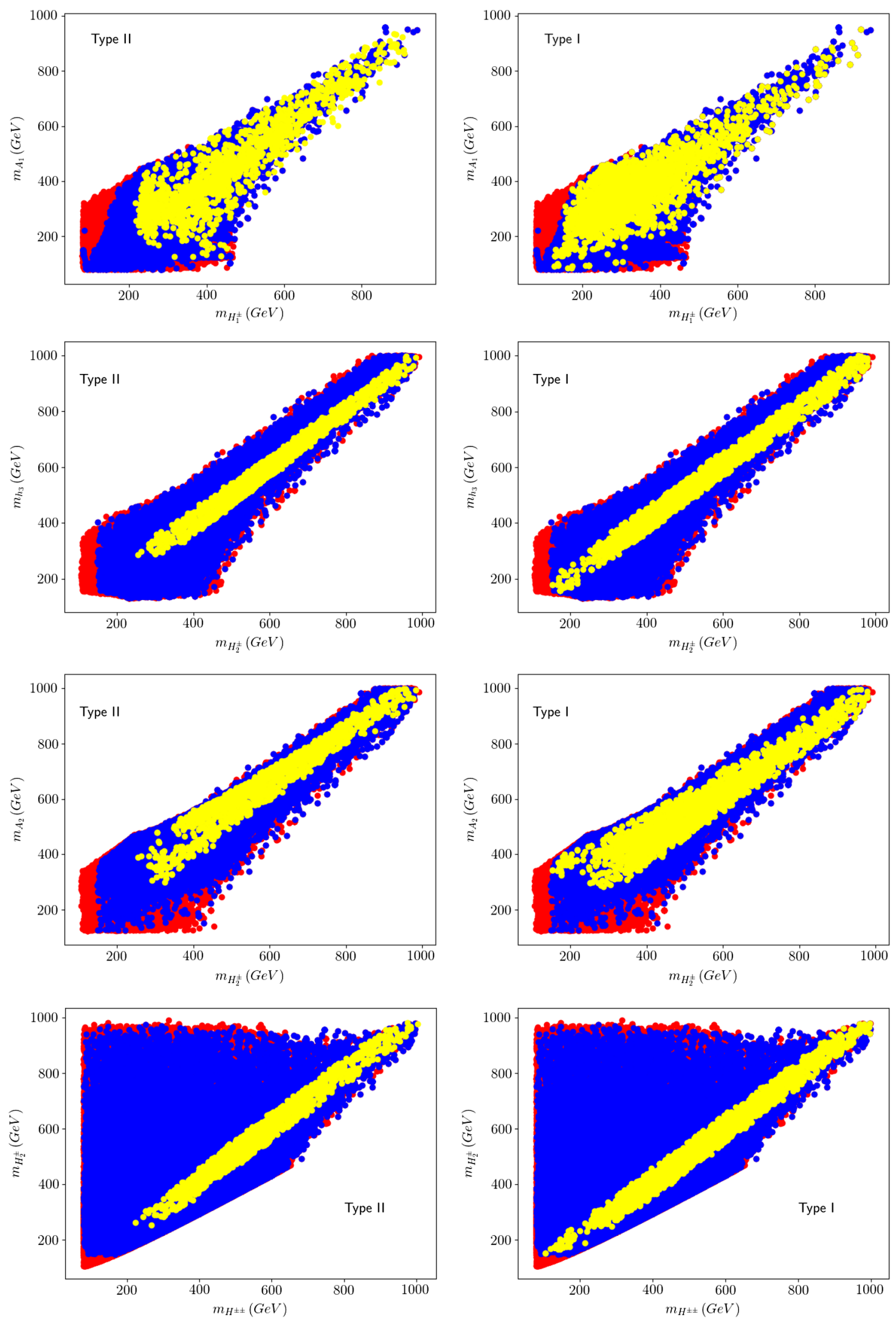

FIG. 6. Allowed mass ranges in 2HDMcT type-II (left column) and type-I (right column) taking into account all theoretical and experimental constraints. 
where $A_{R, L}$ that correspond to $\mu \rightarrow e_{R, L} \gamma$ [36] are given in the 2HDMcT as [37],

$$
A_{R}=-\frac{q_{e}\left(h^{+} h\right)_{e \mu}}{48 \sqrt{2} \pi^{2} G_{F}}\left(\frac{C_{23}}{8 m_{H_{1}^{ \pm}}^{2}}+\frac{C_{33}}{8 m_{H_{2}^{ \pm}}^{2}}+\frac{1}{m_{H^{ \pm \pm}}^{2}}\right),
$$

$$
A_{L}=0
$$

A key point here is that the electron mass in the final state and all lepton masses in the loop are neglected. $q_{e}$ and $C_{i j}$ stand for the electron charge and $\mathcal{C}$ is the rotation matrix elements, respectively. It is worth noting that experimental data put an upper limits for $\mathrm{BR}(\mu \rightarrow \bar{e} e e)<1 \times 10^{-12}$ [38] and $\operatorname{BR}(\mu \rightarrow e \gamma)<4.2 \times 10^{-13}$ [39].

In our study, we consider the two scenarios of neutrino masses hierarchy: (i) normal hierarchy (NH) where $\Delta m_{31}^{2}=m_{3}^{2}-m_{1}^{2}>0$ and (ii) $m_{1}<m_{2}<m_{3}$ and inverted hierarchy (IH) where $m_{3}<m_{1}<m_{2}$. The best-fit parameters that enter in the neutrino mass mixing according to [3] are summarized in Table III.
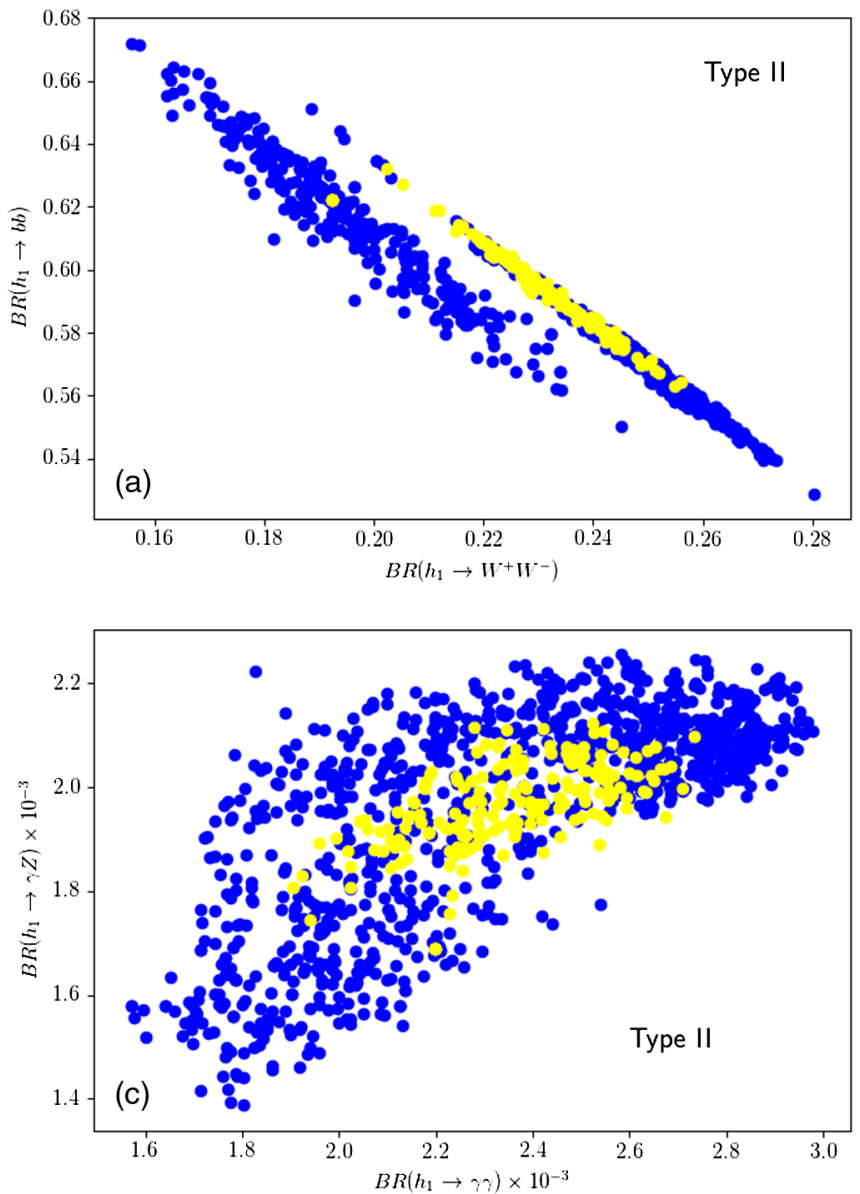

We illustrate in Fig. 2 the lightest neutrino mass $m_{0}$ dependence on $v_{t}$ taking into account best fit values for neutrinos masses parameters. We plot $m_{0}$ mass as a function of $\delta$ for four values of triplet vev both for $\mathrm{NH}$ and IH scenarios.

In the case of $\mathrm{NH}$ (left panel), as one can see, for $\varphi_{1,2}=\pi$ and $1 \mathrm{TeV}$ mass for $H^{ \pm}$and $H^{ \pm \pm}$, large triplet vev requires large $m_{0}$. The dashed area between $\delta$ axis and the solid curve is allowed. Note also that for $\mathrm{NH}$, with $v_{t}=1 \mathrm{eV}, m_{0}$ is constrained to be less than $0.01 \mathrm{eV}$ while for $v_{t}=8 \mathrm{eV} m_{0}$ should be less than $\approx 0.08 \mathrm{eV}$.

In the opposite case of $\mathrm{IH}$, for $v_{t}=4.5 \mathrm{eV}$, neutrino mass becomes tachyonic for $\delta \leq 0.6 \pi$ and $\delta \geq 1.4 \pi$. For $\delta \in[0.6 \pi, 1.4 \pi], m_{0}$ is constrained to be less than $0.01 \mathrm{eV}$. While for smaller triplet vev $v_{t} \leq 4.5 \mathrm{eV}$, neutrino mass becomes tachyonic.

Assuming a situation where $m_{1}=0.06 \mathrm{eV}$ and $1 \mathrm{TeV}$ mass for $H^{ \pm}$and $H^{ \pm \pm}$and setting the Dirac phase $\delta=\pi$, we display in Fig. 3 the allowed space for the Majorana phases $0 \leq \varphi_{1}, \varphi_{2}<2 \pi$ regarding the experimental limits on $\operatorname{BR}(\mu \rightarrow \bar{e} e e)$ and $\operatorname{BR}(\mu \rightarrow e \gamma)$. In the case of $\mathrm{IH}$, and for $v_{t}=5.5 \mathrm{eV}$, both processes could be observed almost
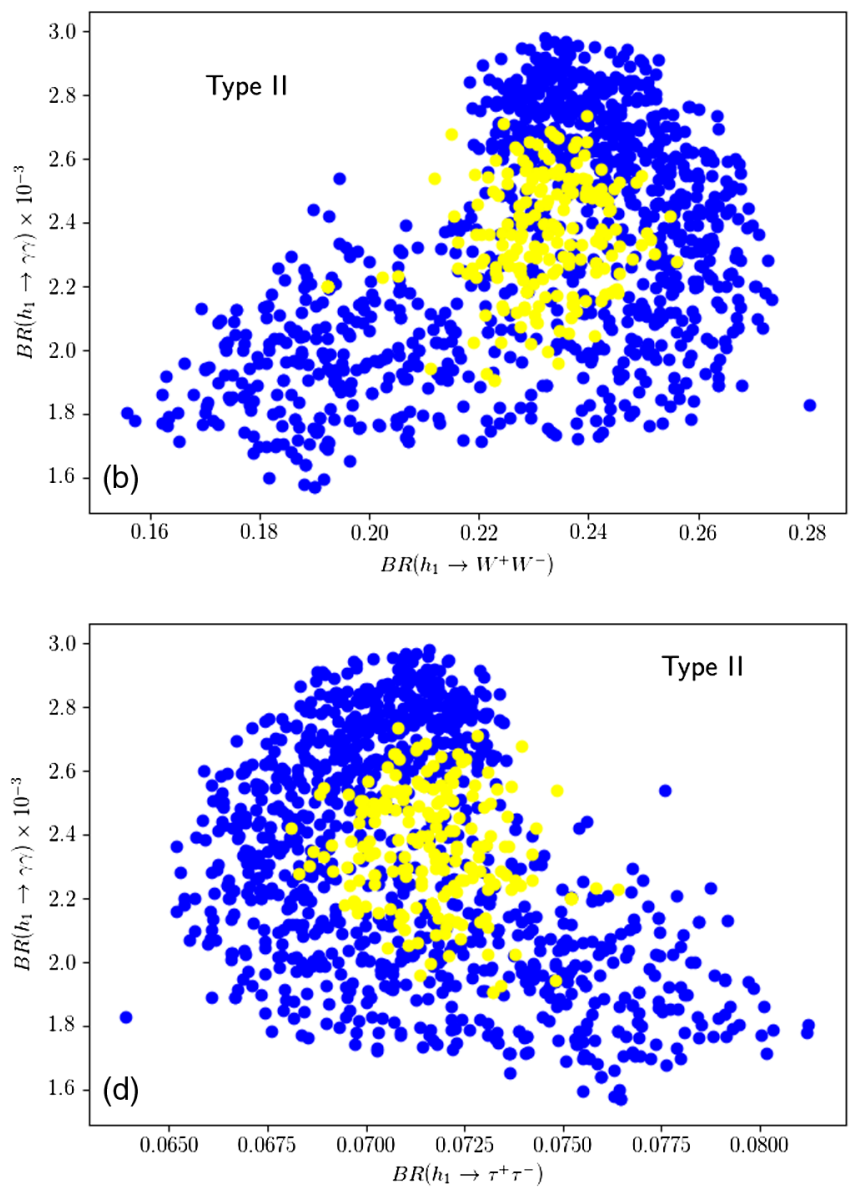

FIG. 7. Correlation plots between the BRs of (a) $h_{1} \rightarrow W W$ vs $h_{1} \rightarrow \gamma \gamma$, (b) $h_{1} \rightarrow W W$ vs $h_{1} \rightarrow b \bar{b}$, (c) $h_{1} \rightarrow \tau \tau$ vs $h_{1} \rightarrow \gamma \gamma$ and (d) $h_{1} \rightarrow \gamma \gamma$ vs $h_{1} \rightarrow h_{1} \rightarrow Z \gamma$ in 2HDMcT type-II by considering the BRs of the lightest $h_{1}$ boson consistent within 95.5\% CL (blue) and $68 \%$ CL (yellow). 
all over the allowed space except for a narrow area where $\varphi_{1} \approx \pi, \varphi_{2} \approx 0[2 \pi]$. For $v_{t} 5 \mathrm{eV}$, one can see that for all values of $\varphi_{2}$ only $\varphi_{1} \in[0.5 \pi, 1.5 \pi]$ is allowed. This range is getting larger for $v_{t}=4$ and $2 \mathrm{eV}$. In the opposite case of $\mathrm{NH}$ with $v_{t}=5 \mathrm{eV}$, one can see that the excluded region for $\phi_{1,2}$ is extended with respect to IH case. A wide region for $\varphi_{1} \in[0.7 \pi, 1.3 \pi]$ is excluded for $\varphi_{2} \in[0,0.6 \pi]$ and $\varphi_{2} \in[1.4 \pi, 2 \pi]$. For smaller $v_{t}$ values we have similar picture as for the IH case.

\section{LIGHT AND HEAVY HIGGS PHENOMENOLOGY}

In this section we study the influence of the constraints presented in the previous section (indirect, LEP, Tevatron and LHC constraints) on the free parameters. For this purpose we generate a set of $10^{9}$ points randomly for each of the two different types of model defined in Tables I and II with random values for each of the free parameters. The available ranges used in our simulation are,
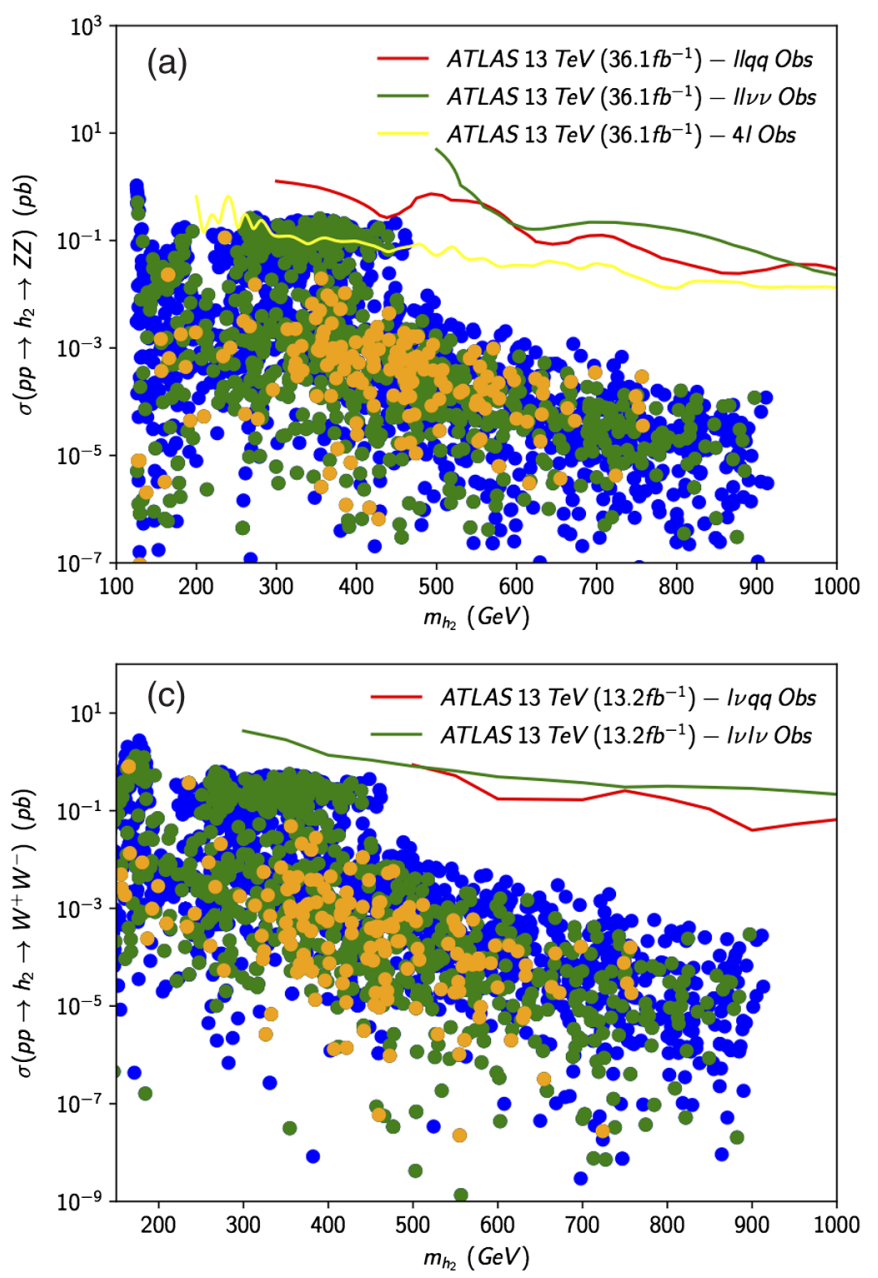

$m_{h_{1}} \leq m_{h_{2}} \leq m_{h_{3}} \leq 1 \mathrm{TeV}, \quad 80 \mathrm{GeV} \leq m_{H^{ \pm \pm}} \leq 1 \mathrm{TeV}$, $\frac{-\pi}{2} \leq \alpha_{1,2,3} \leq \frac{\pi}{2} 1 \leq \tan \beta \leq 40,-10^{2} \leq \mu_{1} \leq 10^{2}, v_{t}=1 \mathrm{GeV}$ $\lambda_{1} \approx 0.15, \quad \lambda_{3} \approx 1.6, \quad \lambda_{4} \approx 1.6$

$\lambda_{1}, \lambda_{3}$, and $\lambda_{4}$ are set respectively to the values $0.15,1.6$, and 1.6 for the sake of simplification. The ranges for $\lambda_{6}, \lambda_{7}$, $\lambda_{8}, \lambda_{9}, \bar{\lambda}_{8}$, and $\bar{\lambda}_{9}$ resulted from the unitarity and boundedness constraints as can be seen from Fig. 4. For simplicity, let us classify the dimensionless parameters in the scalar potential into two different sets according to the following two types of constraints:

(i) First set of constraints includes the unitarity, vacuum stability and BFB constraints as well as nontachyonic masses. We refer to this set as $C_{1}$.

(ii) The second set of constraints contains $C_{1}$ and constraints from Higgs data. We refer to this set as $C_{2}$. In Fig. 4, all the generated points are plotted in the planes $\lambda_{6}$ vs $\lambda_{8}, \lambda_{7}$ vs $\lambda_{9}$, and $\bar{\lambda}_{8}$ vs $\bar{\lambda}_{9}$, as we can see $C_{2}$ set of
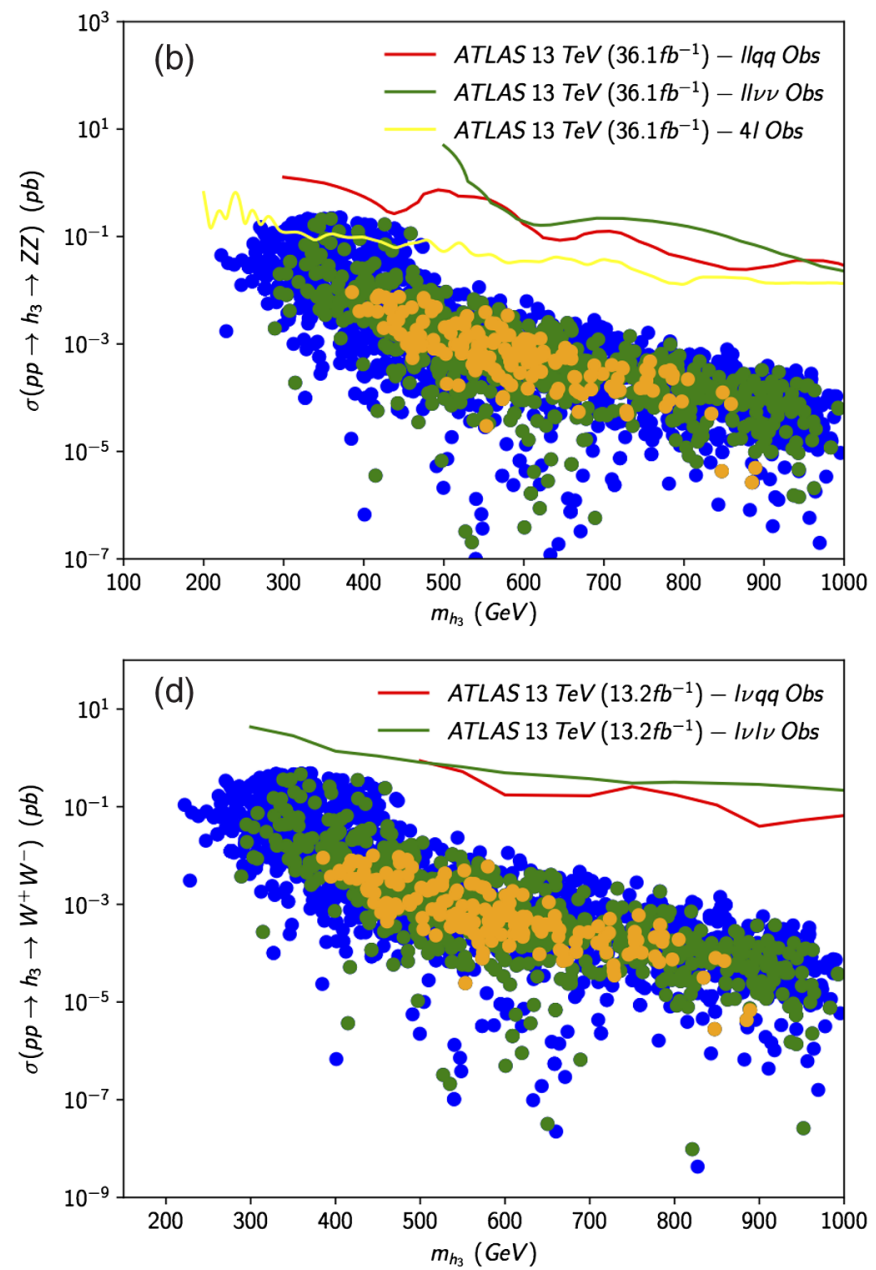

FIG. 8. Scatter plot for $\sigma\left(p p \rightarrow h_{2,3}\right) \times \operatorname{Br}\left(h_{2,3} \rightarrow Z Z, W W\right)$ as a function $m_{h_{2,3}}$ after imposing $C_{1}$ and $C_{2}$ constraints assuming 99.7\% CL (red), 95.5\% CL (blue), and 68\% CL (yellow) in 2HDMcT type-II. 
constraints reduce the above domain of $\lambda_{6}, \lambda_{7}, \lambda_{8}, \lambda_{9}, \bar{\lambda}_{8}$, and $\bar{\lambda}_{9}$.

Looking now at the plane $\bar{\lambda}_{8}$ vs $\bar{\lambda}_{9}$ are not very much restricted by $C_{2}$ constraint due to the fact that $\bar{\lambda}_{8}$ and $\bar{\lambda}_{9}$ are always dependent of the vev of scalar triplet.

In Fig. 5, we present the allowed points in the $\left(\operatorname{sign}\left(C_{V}^{h_{1}}\right) \sin \left(\alpha_{1}-\pi / 2\right), \tan \beta\right)$ plane, that passes all constraints in type-II (left) and type-I (right) at $1 \sigma, 2 \sigma$ and $3 \sigma$. In type-II, one can appreciate that the mixing angle $\alpha_{1}$ seems more constrained than in type-I. Results are shown by imposing the conditions $C_{1}$ and $C_{2}$. The latter has a strong impact on how the mixing angles are constrained. Fig. 5(left) displays wrong-sign Yukawa couplings scenario at $2 \sigma$.

In Fig. 6, the allowed ranges are plotted in the planes $m_{A_{1}}, m_{A_{2}}, m_{h_{3}}$, and $m_{H^{ \pm \pm}}$vs $m_{H_{2}^{ \pm}}$. The left-columns panels corresponds to type-II, the right-column to the type-I, all points passed the constraints mentioned above at $1 \sigma$ (yellow), $2 \sigma$ (blue) and $3 \sigma$ (red). As can be seeing most of the masses can be light in type-I less than $300 \mathrm{GeV}$.
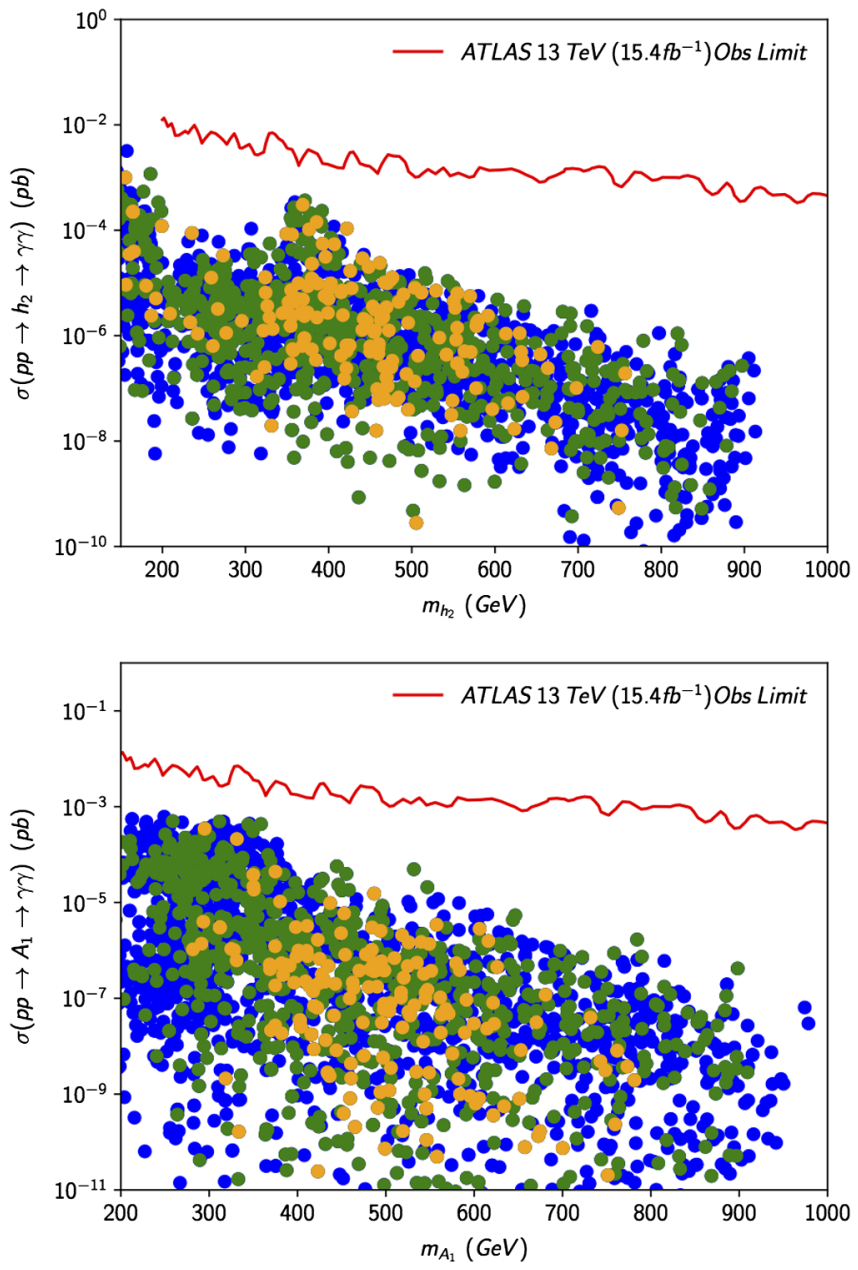

Looking only at the yellow points, those which pass the $C_{2}$ constraint, we can see that the $m_{A_{2}}, m_{H_{2}^{ \pm}}, m_{h_{3}}$ and $m_{H^{ \pm \pm}}$masses are bounded in type-II where we find that most of the yellow points lie in the ranges $m_{H_{1}^{ \pm}, H_{2}^{ \pm}, A_{1}, A_{2}, h_{2}, h_{3}, H^{ \pm \pm}} \in[200,1000] \mathrm{GeV}$.

The sensitivity of the Higgs couplings of $h_{1}$ at the LHC is not appreciably better than $20 \%$, leaving thus a significant window of opportunity for new physics. Here we investigate the correlations among relevant couplings within the $C 1$ and $C_{2}$ constraints. In Fig 7, we show these correlations which are consistent within the BRs of $m_{h_{1}}=$ $125 \mathrm{GeV}$ within $1 \sigma$ (yellow) and $2 \sigma$ (blue). This is related to the fact that the central values of some Higgs couplings deviate from the SM, which strongly restrict the range of deviations from the SM. While the decay $h_{1} \rightarrow Z \gamma$ is not yet observed at the LHC, the correlation between the $h_{1} \rightarrow W W, \gamma \gamma$ and $h \rightarrow b \bar{b}$ couplings can now be measured by the experiments. The ratio of the BR of $W W$ to that $\gamma \gamma$ can be measured with an accuracy better than $5 \%$ and an integrated luminosity that is expected to be
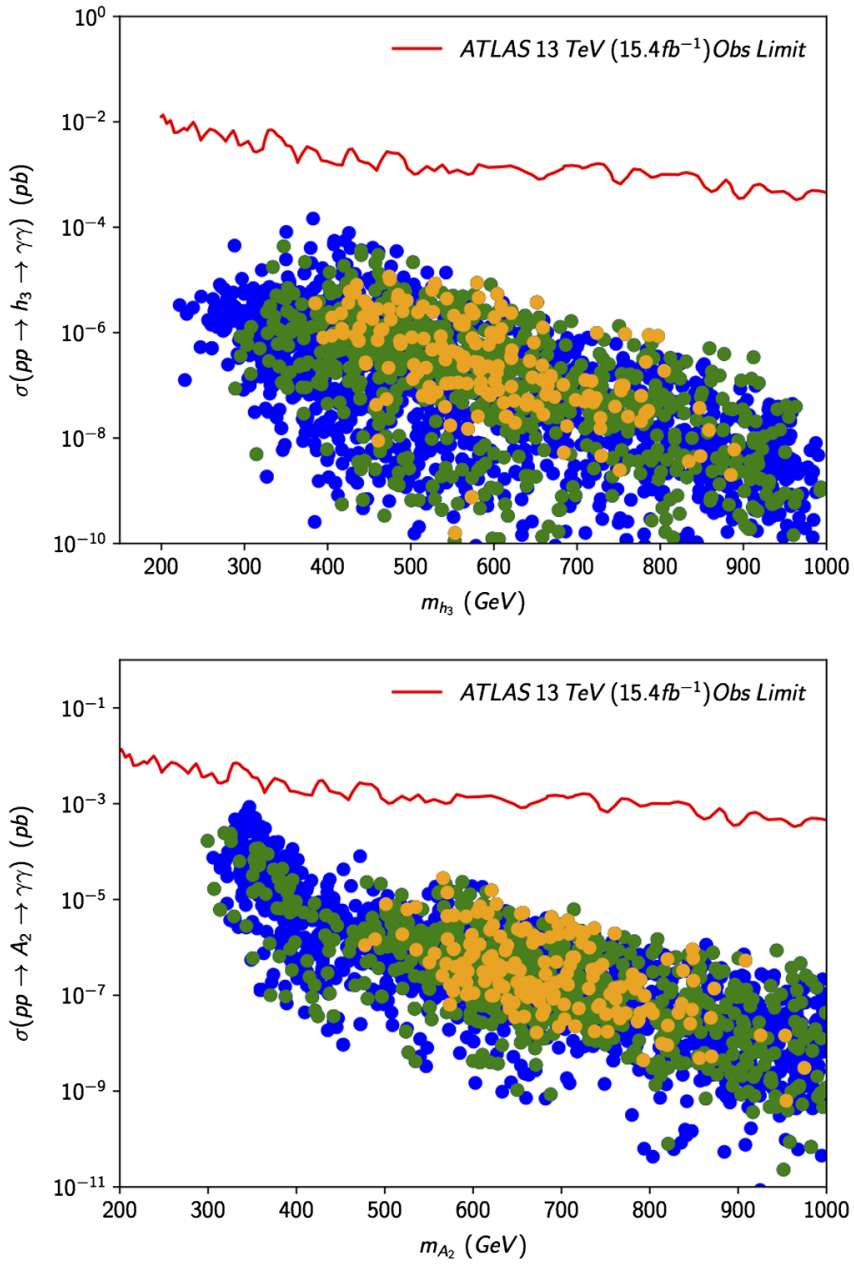

FIG. 9. Scatter plot in the $m_{\phi^{-}}[\sigma \times \operatorname{Br}(\phi \rightarrow \gamma \gamma)]\left(\phi=h_{2,3}\right.$ or $\left.A_{2,3}\right)$ planes after imposing $C_{3}$ constraints at $\sqrt{s}=13$ TeV. The errors for $\chi$-square fit are $99.7 \% \mathrm{CL}$ (red), $95.5 \% \mathrm{CL}$ (blue), and 68\% CL (yellow) in 2HDMcT type-II. 
accumulated by the High Luminosity LHC. In the following, we will scrutinize the impact of the searches for heavy Higgs particles in 2 HDMcT ordered by their decay products. First we will address the bosonic decays to $V V$ $\left(V=\gamma, Z, W^{ \pm}\right)$and fermionic mode $\tau^{-} \tau^{+}$branching ratios of the neutral Higgs $\left(h_{2}, h_{3}, A_{1}\right.$ and $\left.A_{2}\right)$ using both CMS and ATLAS Higgs data for $8 \mathrm{TeV}$ [40-42] and $13 \mathrm{TeV}$ [43-47]. After that, we will turn towards the pair production of $h_{i} h_{j}$. The narrow width approximation will be applied throughout this section, we will comment on its validity at the end of the text section. We define the cross section as,

$\sigma^{2 H D M c T}(p p \rightarrow \phi)=k_{g}^{2} \sigma^{\mathrm{SM}}(p p \rightarrow \phi), \quad \phi=h_{2,3}$ or $A_{1,2}$

where $\sigma^{\mathrm{SM}}(g g \rightarrow \phi)$ is the cross section for Higgs production in gluon fusion in the $\mathrm{SM}$, and $k_{g}^{2}=$ $\Gamma_{2 H D M c T}(\phi \rightarrow g g) / \Gamma_{\mathrm{SM}}(\phi \rightarrow g g)$, with $\Gamma_{2 H D M c T}(\phi \rightarrow g g)$ and $\Gamma_{\mathrm{SM}}(\phi \rightarrow g g)$ are the partial decay rates in 2HDMcT and SM, respectively.
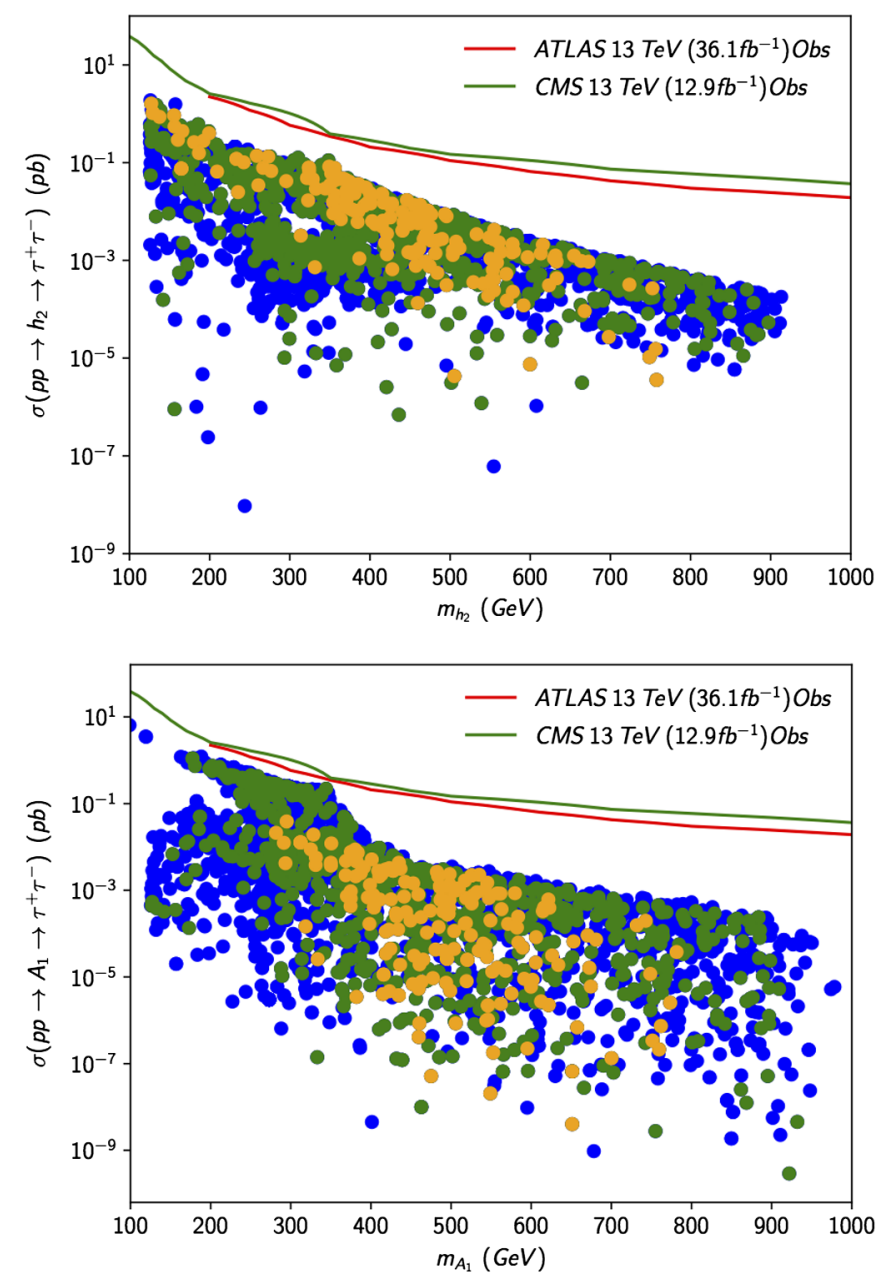

\section{A. $h_{2,3} \rightarrow Z Z$ and $W W$}

We use Sushi $v 1.6 .0$ public code $[48,49]$ at NNLO QCD to perform the calculation of the cross sections for Higgs production in gluon fusion $(g g F)$ and bottom-quark annihilation in the SM at $13 \mathrm{TeV}$. The relative coupling of $h_{2,3}$ to the two vector bosons $Z Z$ and $W W$ is universal and type independent. However, the production of the $h_{2,3}$ differs between the types. In Fig. 8 we plot the production cross sections in proton proton fusion times $Z Z$ and $W W$ branching ratios of $h_{2,3}$ Higgs after imposing $C_{1}$ and $C_{2}$ constrains within $99.7 \% \mathrm{CL}$ (red), $95.5 \% \mathrm{CL}$ (blue), and $68 \% \mathrm{CL}$ (yellow) of the Higgs data. It is well seeing from Fig. 8 that $h_{2,3} \rightarrow W W / Z Z$ rates are compared to what would be expected at Run-II LHC. The colored lines in Fig. 8 denote the observed limits in $Z Z / W W$ final states from $13 \mathrm{TeV}$ ATLAS data [50-53]. The direct LHC searches for this channels yield a strong suppression of $\sigma \times \mathrm{BR}$. For $Z Z$ searches the $m_{h_{2}} \leq 250 \mathrm{GeV}$ region is constrained by Run-I data whereas Run-II data determine the dominant limits for the rest of the mass region. For the $W W$ searches, Run-I data dictate the limit until
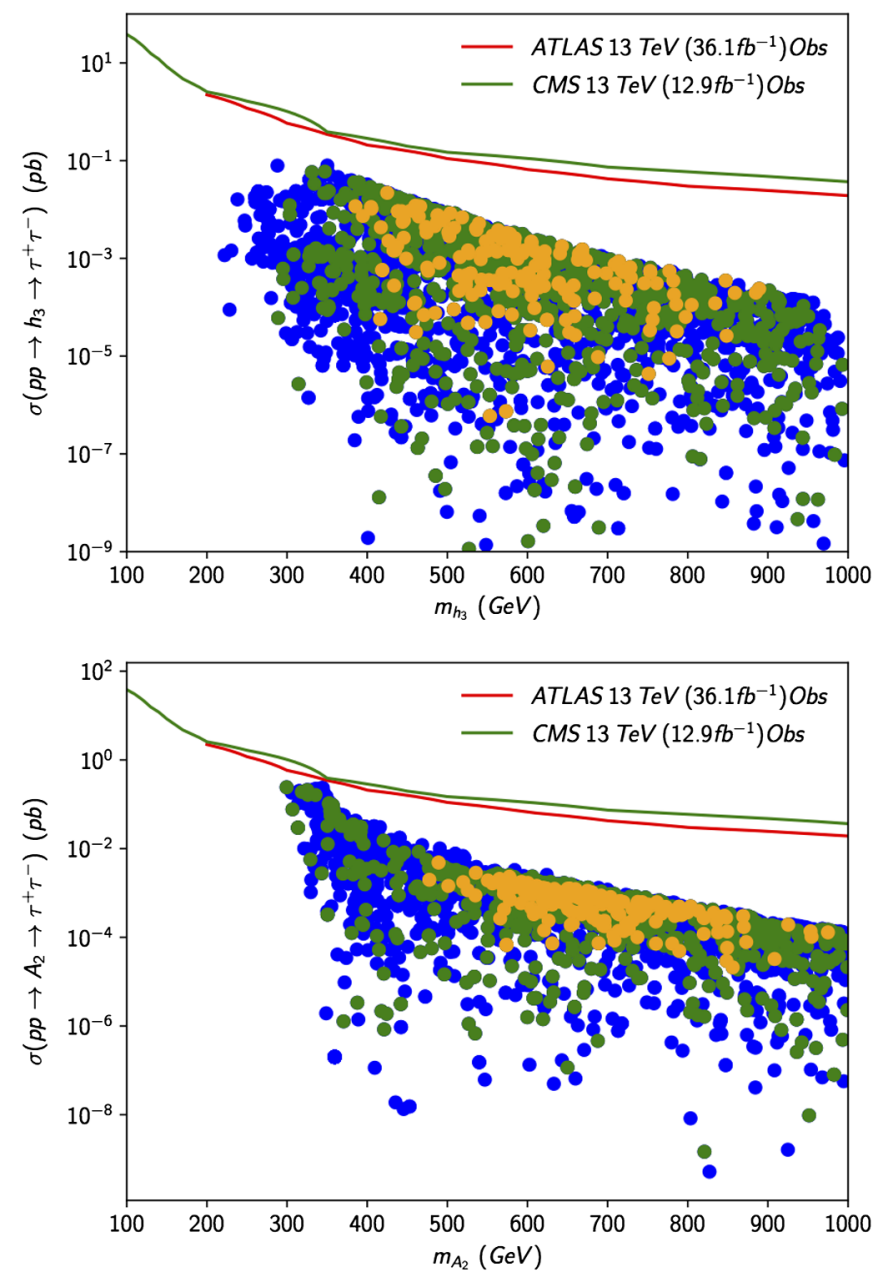

FIG. 10. The same as Fig. 9 but for $\tau \tau$ pair production at $\sqrt{s}=13 \mathrm{TeV}$ in 2HDMcT type-II. 
$350 \mathrm{GeV}$ and the high mass range is dominated by Run-II data.

$$
\text { B. } h_{2,3}, A_{1,2} \rightarrow \gamma \gamma
$$

Direct searches for a heavy Higgs decaying to two photons constrain $\sigma \times \mathrm{BR}$ by roughly one magnitude compared the ATLAS limit in $C P$-even decay modes (see Fig. 9). The searches in the diphoton decay channel of a pseudoscalar Higgs yield a suppression of $\sigma \times \mathrm{BR}$ one to three orders of magnitude compared to the ATLAS limit for $m_{A_{1,2}} \leq 400 \mathrm{GeV}$ certain intermediate $\sigma \times \mathrm{BR}$ regions for low $m_{A_{1,2}}$ are disfavoured by the prior.

In Fig. 10 we plot the production cross section in proton proton fusion times $\tau \tau$ branching ration after imposing $C_{3}$ constrains. The errors for $\chi$-square fit are 99.7\% CL (red), 95.5\% CL (blue), and 68\% CL (yellow). The red (green) solid line in Fig. 10 is the upper limit on the cross section times branching ration from the ATLAS
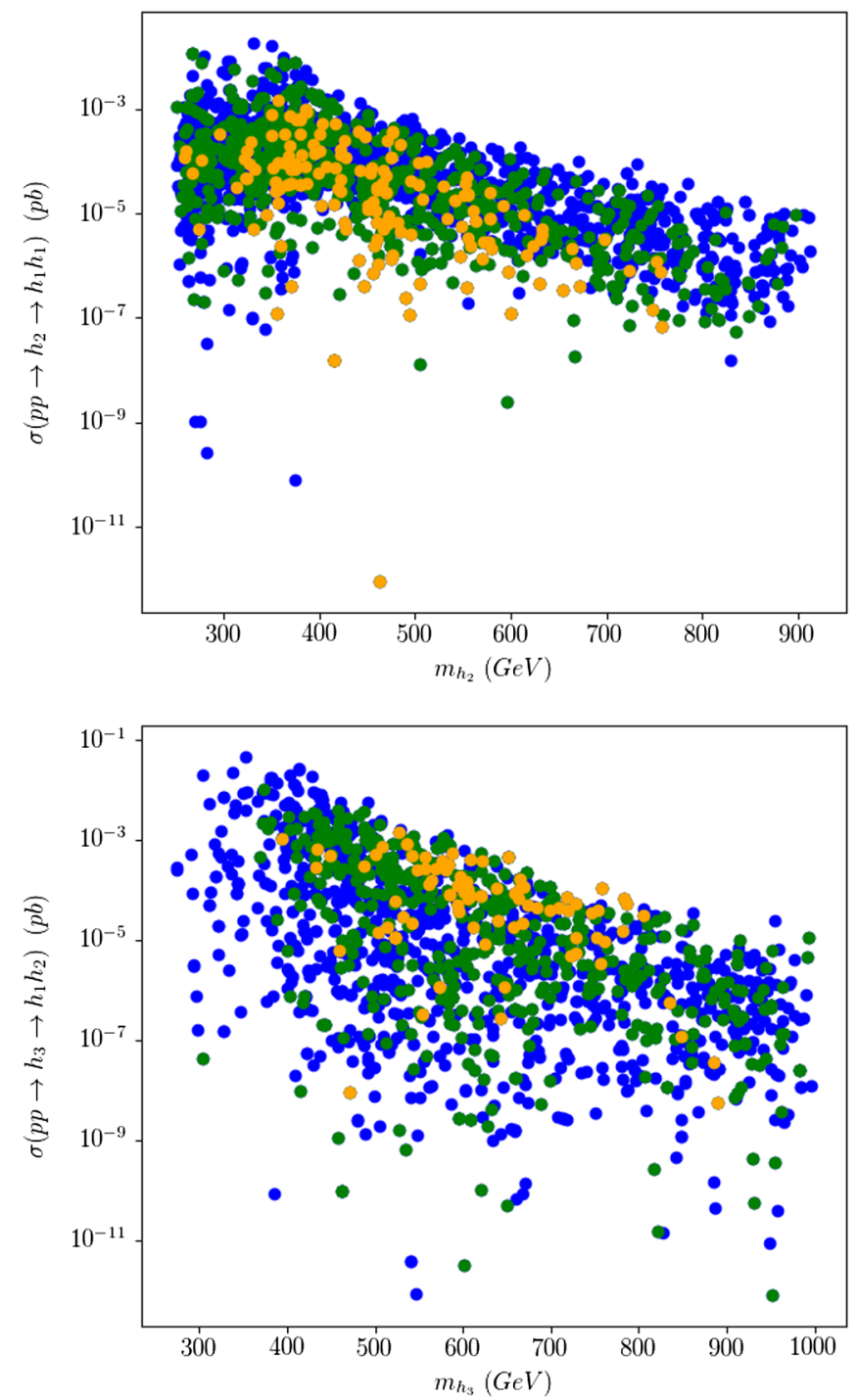

$13 \mathrm{TeV}$ results [54] (CMS $13 \mathrm{TeV}$ results [55]). The colored lines in Fig. 9 denote the observed limits from $13 \mathrm{TeV}$ ATLAS data [56].

$$
\text { C. } h_{2,3} \rightarrow h_{i} h_{j} \text { with }(i, j)=(1,2)
$$

Finally, in Fig. 11 we present a sample of points generated for Higgs pair production in the scenario where the observed Higgs boson is the lightest scalar $h_{1}$ (top panels) and the next-to-lightest scalar (bottom panels). We present cross sections as a function of the masses of the two new scalars that can be involved in the chain decay contributions. We overlay three layers of point for which the total cross section is within $3 \sigma$ (red), $2 \sigma$ (blue), and $1 \sigma$ respectively at the LHC Run-2. In the upper plots, where $h_{1}$ chain decays become possible above $250 \mathrm{GeV}$. In Fig. 11 at nearly $m_{h_{1}} \sim m_{h_{2}}$, which means that some opening decay channels contribute to chain decay $h_{3} \rightarrow h_{1}+h_{1}, h_{1}+h_{2}$ and $h_{2}+h_{2}$. This means that imposing a constraint on the
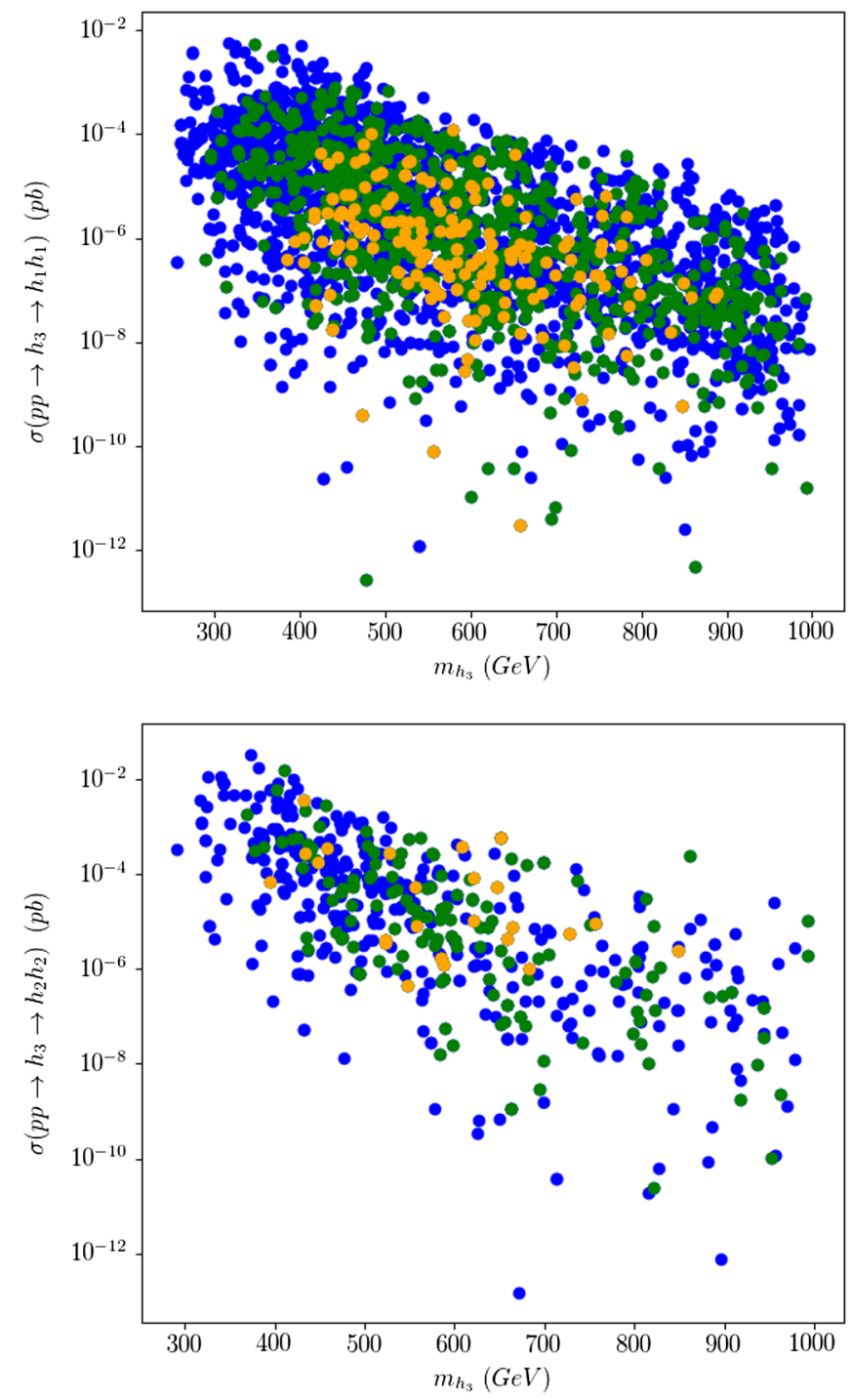

FIG. 11. The same as Fig. 9 but for $h_{i} h_{j}$ pair production at $\sqrt{s}=13 \mathrm{TeV}$ in 2HDMcT type-II. 
parameter space with including the chain decay contribution would be too strong at the LHC Run-2. Overall, the shape of the three different $\sigma$ regions in the various panels is the results of an interplay between the kinematics and the applied constraints. To a certain extent the structure can be directly related to the exclusion curves from the collider searches imposed by HiggsBounds. The strong increase at $m_{h_{3}}=250 \mathrm{GeV}$ in the lower left panel is due the opening

TABLE IV. Benchmark points for LHC Run-2 in 2HDMcT. The cross sections are for $\sqrt{s} \equiv 13 \mathrm{TeV}$.

\begin{tabular}{|c|c|c|c|c|c|}
\hline & BP1 & BP2 & BP3 & BP4 & BP5 \\
\hline$m_{h 1}(\mathrm{GeV})$ & 125.1 & 125.1 & 12.75 & 77.27 & 21.8 \\
\hline$m_{h_{2}}(\mathrm{GeV})$ & 415.46 & 354.18 & 125.1 & 125.1 & 101.7 \\
\hline$m_{h_{3}}(\mathrm{GeV})$ & 426.17 & 937.02 & 155.96 & 448.59 & 125.1 \\
\hline$m_{A_{1}}(\mathrm{GeV})$ & 258.76 & 311.72 & 152.46 & 174.34 & 104 \\
\hline$m_{A_{2}}(\mathrm{GeV})$ & 417.81 & 936.06 & 270.28 & 457 & 327 \\
\hline$m_{H_{1}^{ \pm}}(\mathrm{GeV})$ & 270.22 & 267.60 & 147.13 & 232.8 & 143 \\
\hline$m_{H_{2}^{ \pm}}(\mathrm{GeV})$ & 386.35 & 906.42 & 164.24 & 434.7 & 174.87 \\
\hline$m_{H^{ \pm \pm}}(\mathrm{GeV})$ & 352.06 & 875.54 & 161.01 & 397.2 & 174.89 \\
\hline$\alpha_{1}$ & -1.26 & -1.25 & -0.46 & -0.58 & -0.08 \\
\hline$\alpha_{2}$ & -0.011 & -0.086 & -0.16 & 0.45 & -0.06 \\
\hline$\alpha_{3}$ & -1.08 & -0.12 & -0.13 & -0.08 & -1.25 \\
\hline$v_{t}(\mathrm{GeV})$ & 1 & 1 & 1 & 1 & 1 \\
\hline$\lambda_{6}$ & 5.56 & 0.89 & 6.24 & 4.17 & 5.92 \\
\hline$\lambda_{7}$ & 1.13 & 0.69 & 5.52 & 4.57 & 7.08 \\
\hline$\lambda_{8}$ & 2.75 & 1.54 & -2.92 & 1.5 & -4.64 \\
\hline$\lambda_{9}$ & 1.50 & 3.36 & 0.90 & -1.04 & -0.6 \\
\hline $\tan \beta$ & 2.57 & 2.53 & 1.7 & 1.07 & 8.83 \\
\hline$\mu_{1}$ & 26.22 & 16.42 & -21.49 & -36 & 56.69 \\
\hline$\mu_{2}$ & 11.9 & 263.7 & 6.19 & -451 & 1.19 \\
\hline$\mu_{3}$ & -28.95 & -615.2 & 3.41 & 525.42 & -14.69 \\
\hline$\underline{\mu_{h_{1}}}$ & 0.88 & 0.86 & 0.043 & 0.069 & 0.78 \\
\hline$\sigma_{1} \equiv \sigma\left(g g \rightarrow h_{1}\right)$ & $132.9[\mathrm{pb}]$ & $131.7[\mathrm{pb}]$ & $10003.81[\mathrm{pb}]$ & $175.45[\mathrm{pb}]$ & $423.9[\mathrm{pb}]$ \\
\hline$\sigma_{1} \times \mathrm{BR}\left(h_{1} \rightarrow W W\right)$ & 11.69 [pb] & $10.70[\mathrm{pb}]$ & $0[\mathrm{fb}]$ & $0[\mathrm{fb}]$ & $0[\mathrm{pb}]$ \\
\hline$\sigma_{1} \times \mathrm{BR}\left(h_{1} \rightarrow Z Z\right)$ & $1.73 \times 10^{3}[\mathrm{fb}]$ & $1.59 \times 10^{3}[\mathrm{fb}]$ & $0[\mathrm{fb}]$ & $0[\mathrm{fb}]$ & $0[\mathrm{pb}]$ \\
\hline$\sigma_{1} \times \mathrm{BR}\left(h_{1} \rightarrow b b\right)$ & $35.1[\mathrm{pb}]$ & $35.96[\mathrm{pb}]$ & $4811[\mathrm{pb}]$ & $71.62[\mathrm{pb}]$ & $177.45[\mathrm{pb}]$ \\
\hline$\sigma_{1} \times \mathrm{BR}\left(h_{1} \rightarrow \tau \tau\right)$ & $4.24[\mathrm{pb}]$ & $4.35[\mathrm{pb}]$ & $452 \times 10^{3}[\mathrm{fb}]$ & $7.8 \times 10^{3}[\mathrm{fb}]$ & $17.7 \times 10^{3}[\mathrm{fb}]$ \\
\hline$\sigma_{1} \times \mathrm{BR}\left(h_{1} \rightarrow \gamma \gamma\right)$ & $0.12 \times 10^{3}[\mathrm{fb}]$ & $0.12 \times 10^{3}[\mathrm{fb}]$ & $7[\mathrm{fb}]$ & $8.4[\mathrm{fb}]$ & $0.34[\mathrm{fb}]$ \\
\hline$\underline{\mu_{h_{2}}}$ & 0.03 & 0.095 & 0.78 & 0.6 & 0.01 \\
\hline$\sigma_{2} \equiv \sigma\left(g g \rightarrow h_{2}\right)$ & $0.53[\mathrm{pb}]$ & $4.74[\mathrm{pb}]$ & $106.18[\mathrm{pb}]$ & $94.83[\mathrm{pb}]$ & $18.31[\mathrm{pb}]$ \\
\hline$\sigma_{2} \times \mathrm{BR}\left(h_{2} \rightarrow W W\right)$ & $0.11[\mathrm{pb}]$ & $0.56[\mathrm{pb}]$ & $9.55 \times 10^{3}[\mathrm{fb}]$ & $11.4 \times 10^{3}[\mathrm{fb}]$ & $4.98 \times 10^{-3}[\mathrm{pb}]$ \\
\hline$\sigma_{2} \times \mathrm{BR}\left(h_{2} \rightarrow Z Z\right)$ & $52[\mathrm{fb}]$ & $260[\mathrm{fb}]$ & $1.41 \times 10^{3}[\mathrm{fb}]$ & $1.69 \times 10^{3}[\mathrm{fb}]$ & $9.66 \times 10^{-5}[\mathrm{pb}]$ \\
\hline$\sigma_{2} \times \mathrm{BR}\left(h_{2} \rightarrow b b\right)$ & $6.2 \times 10^{-4}[\mathrm{pb}]$ & $4.1 \times 10^{-3}[\mathrm{pb}]$ & $19.06[\mathrm{pb}]$ & $23.12[\mathrm{pb}]$ & $0.35[\mathrm{pb}]$ \\
\hline$\sigma_{2} \times \mathrm{BR}\left(h_{2} \rightarrow \tau \tau\right)$ & $9.38 \times 10^{-5}[\mathrm{pb}]$ & $6.1 \times 10^{-4}[\mathrm{pb}]$ & $2.3 \times 10^{3}[\mathrm{fb}]$ & $2.79 \times 10^{3}[\mathrm{fb}]$ & $0.041 \times 10^{3}[\mathrm{fb}]$ \\
\hline$\sigma_{2} \times \mathrm{BR}\left(h_{2} \rightarrow \gamma \gamma\right)$ & $7.401 \times 10^{-4}[\mathrm{fb}]$ & $7.86 \times 10^{-3}[\mathrm{fb}]$ & $0.1 \times 10^{3}[\mathrm{fb}]$ & $0.08 \times 10^{3}[\mathrm{fb}]$ & $1.86[\mathrm{fb}]$ \\
\hline $\mathrm{BR}\left(h_{2} \rightarrow h_{1} h_{1}\right) \%$ & $5.1 \times 10^{-2}$ & $1.6 \times 10^{-2}$ & 25.69 & 0 & 94.68 \\
\hline$\mu_{h_{3}}$ & 0.11 & 0.0003 & $4.69 \times 10^{-5}$ & 0.51 & 1.07 \\
\hline$\sigma_{3} \equiv \sigma\left(g g \rightarrow h_{3}\right)$ & $1.43[\mathrm{pb}]$ & $4.6 \times 10^{-4}[\mathrm{pb}]$ & $0.15[\mathrm{pb}]$ & $2.79[\mathrm{pb}]$ & $93.87[\mathrm{pb}]$ \\
\hline$\sigma_{3} \times \mathrm{BR}\left(h_{3} \rightarrow W W\right)$ & $0.35[\mathrm{pb}]$ & $1.52 \times 10^{-6}[\mathrm{pb}]$ & 7 [fb] & $2.4[\mathrm{fb}]$ & $8.86[\mathrm{pb}]$ \\
\hline$\sigma_{3} \times \mathrm{BR}\left(h_{3} \rightarrow Z Z\right)$ & $1.6 \times 10^{2}[\mathrm{fb}]$ & $1.52 \times 10^{-3}[\mathrm{fb}]$ & $0.8[\mathrm{fb}]$ & $2.2[\mathrm{fb}]$ & $1.32[\mathrm{pb}]$ \\
\hline$\sigma_{3} \times \mathrm{BR}\left(h_{3} \rightarrow b b\right)$ & $1.8 \times 10^{-3}[\mathrm{pb}]$ & $2.7 \times 10^{-8}[\mathrm{pb}]$ & $0.004[\mathrm{pb}]$ & $4.2 \times 10^{-4}[\mathrm{pb}]$ & $19.14[\mathrm{pb}]$ \\
\hline$\sigma_{3} \times \mathrm{BR}\left(h_{3} \rightarrow \tau \tau\right)$ & $2.7 \times 10^{-4}[\mathrm{pb}]$ & $4.6 \times 10^{-9}[\mathrm{pb}]$ & $0.56[\mathrm{fb}]$ & $6.46 \times 10^{-2}[\mathrm{fb}]$ & $2.31[\mathrm{fb}]$ \\
\hline$\sigma_{3} \times \mathrm{BR}\left(h_{3} \rightarrow \gamma \gamma\right)$ & $2.027 \times 10^{-3}[\mathrm{fb}]$ & $2.25 \times 10^{-8}[\mathrm{fb}]$ & $1.54 \times 10^{-3}[\mathrm{fb}]$ & $5.05 \times 10^{-3}[\mathrm{fb}]$ & $0.14[\mathrm{fb}]$ \\
\hline $\mathrm{BR}\left(h_{3} \rightarrow \sum_{i=1,2} h_{i} h_{j}\right) \%$ & $6.9 \times 10^{-2}$ & $6.1 \times 10^{-2}$ & 81.3 & 0.79 & 18.42 \\
\hline
\end{tabular}


of the decay $h_{3} \rightarrow h_{2}+h_{2}$ and the decrease in the number of points for large values of $m_{h_{3}}$ is due the $\mathrm{S}$ and $\mathrm{T}$ parameters.

\section{BENCHMARK POINTS FOR THE LHC RUN-2 IN 2HDMCT}

Benchmark points. In this section we provide a set of benchmark points in 2HDMcT. There are chosen to cover various physical situations. From a phenomenological perspective we are interested in maximizing various visibility of the new scalars in the LHC Run-2 and in covering, simultaneously, many kinematically different possibilities. In particular, we are interested in scenarios where $h_{\mathrm{SM}}=h_{1}, h_{\mathrm{SM}}=h_{2}$ and $h_{\mathrm{SM}}=h_{3}$ while preserving consistency with LHC Run-2 measurements. Thus, in many of the points presented below we have tried to maximize the cross section for the cross sections. At the same time we required the rates of the SM-like Higgs within $2 \sigma$ from the global signal strength provided by the ATLAS and CMS data from LHC Run-2. Table IV, contains the parameters that define the chosen benchmark points and the production rate of the lightest as well as nextto-lightest Higgs bosons $h_{2}$ and $h_{3}$ in the various final states.

We also give the signal rates $\mu_{h_{i}}(i=1,2,3)$ we have chosen: two points where the SM-like Higgs is the lightest Higgs boson (BP1 and BP2) two points where it is the nextto-lightest Higgs boson (BP3 and BP4) and one point where it is the heaviest (BP5).

Most points were chosen such that the cross sections for the indirect decay channels of the new scalars can compete with the direct decays. In particular we have tried to maximize $h_{3} \rightarrow h_{1}+h_{2}$ where all decays scalars could be observed at once. We have furthermore chosen points with large cross sections for the new scalars, so that they can be detected directly in their decays. In BP1, the lightest Higgs boson is $h_{1}$ and decay channel $h_{3} \rightarrow h_{2}+h_{2}$ is eventually closed. The presented point has a maximum cross section so that all new scalars can be expected to be observed. In BP2, the SM Higgs-like still $h_{1}$ but we allow $h_{3} \rightarrow h_{2}+h_{2}$ now is open with a large possible branching ratio. For the BP3 where $h_{2}$ is the SM-like Higgs boson and $m_{h_{1}}<m_{h_{2}} / 2$, all kinematic situations for the scalar decays are available while the spectrum remains light. So that Higgs-Higgs decays present an interesting discovery option for the heavy Higgs states. Furthermore, large production cross sections have been required for the new light scalar $h_{1}$ so it will be visible in direct decays in addition to chain production from heavier scalars. With BP4, the situation is different from the previous one, so that the channel $h_{2} \rightarrow h_{1}+h_{1}$ is kinematically closed. At the same time the direct $h_{1}$ production rates are increased. Lastly, in BP5 benchmark the spectrum is very light. So that the production of $h_{1}$ is possible through $h_{3} \rightarrow h_{1}+h_{1}$ or $h_{3} \rightarrow h_{1}+h_{2}$. This benchmark point has been required to have large branching ratios for $h_{1} h_{1}$ and $h_{1} h_{2}$, however the direct production rates of the heavier $h_{2}$ are expected to be small, but still accessible at the LHC Run-2.

\section{SUMMARY}

In this paper we presented a comprehensive analysis of the scalar sector in 2HDMcT, when the bosons mix with arbitrary angle $\alpha_{i}(i=1,2,3)$. Of the bare states in the model, one is usual neutral component of the SM Higgs doublet. We started by presenting the model and the theoretical constraints that were imposed. Indeed we have discussed the questions of unitarity and vacuum stability in this model and showed the allowed parameter ranges. We have shown that, in the case of vanishing new extra parameters, the spectrum of the scalar Higgs is reduced to HTM. Later on we studied the phenomenological aspects of the scalar sector by imposing both theoretical and experimental constraints from combined LHC results by identifying the lighter of them with the 125 Higgs boson of standard model. In the numerical analysis, we first investigated how important the contributions from Higgs to gauge bosons decays can be compared to its direct production and decay. Depending on the mixing angles and the scenarios, the production rates can attain a maximum values ranging between $0.1 \mathrm{fb}^{-1}$ and $100 \mathrm{fb}^{-1}$ while remaining compatible with the Higgs signal measurements within $2 \sigma$. We have tested the main chain decays of heavy Higgs bosons scenario where the power to discriminate them from other extended models like 2HDM depends critically on differentiating their couplings and decays.

\section{ACKNOWLEDGMENTS}

This work is supported by Moroccan Ministry of Higher Education and Scientific Research MESRSFC/CNRST, Project No. PPR/2015/6. Part of this work was also done within H2020-MSCA-RISE-2014, Project No. 645722 (NoninimalHiggs). R. B. thanks Uppsala University for the warm hospitality.

\section{APPENDIX A: DIAGONALIZATION MATRICES}

The rotation between the physical and nonphysical states are given by:

$$
\left(\begin{array}{c}
G^{ \pm} \\
H_{1}^{ \pm} \\
H_{2}^{ \pm}
\end{array}\right)=\mathcal{C}\left(\begin{array}{c}
\phi_{1}^{ \pm} \\
\phi_{2}^{ \pm} \\
\delta^{ \pm}
\end{array}\right) \text {and }\left(\begin{array}{c}
G^{0} \\
A_{1} \\
A_{2}
\end{array}\right)=\mathcal{O}\left(\begin{array}{l}
\eta_{1} \\
\eta_{2} \\
\eta_{0}
\end{array}\right)
$$

The matrices elements for each sector are shown below. 
In terms of the mixing angles $\theta_{i}^{ \pm}$

\section{Charged sector}

$$
\mathcal{C}=\left(\begin{array}{lll}
\mathcal{C}_{11} & \mathcal{C}_{12} & \mathcal{C}_{13} \\
\mathcal{C}_{21} & \mathcal{C}_{22} & \mathcal{C}_{23} \\
\mathcal{C}_{31} & \mathcal{C}_{32} & \mathcal{C}_{33}
\end{array}\right)=\left(\begin{array}{ccc}
c_{\theta_{1}^{ \pm}} c_{\theta_{2}^{ \pm}} & s_{\theta_{1}^{ \pm}} c_{\theta_{2}^{ \pm}} & s_{\theta_{2}^{ \pm}} \\
-\left(c_{\theta_{1}^{ \pm}} s_{\theta_{2}^{ \pm}} s_{\theta_{3}^{ \pm}}+s_{\theta_{1}^{ \pm}} c_{\theta_{3}^{ \pm}}\right) & c_{\theta_{1}^{ \pm}} c_{\theta_{3}^{ \pm}}-s_{\theta_{1}^{ \pm}} s_{\theta_{2}^{ \pm}} s_{\theta_{3}^{ \pm}} & c_{\theta_{2}^{ \pm}} s_{\theta_{3}^{ \pm}} \\
-c_{\theta_{1}^{ \pm}} s_{\theta_{2}^{ \pm}} c_{\theta_{3}^{ \pm}}+s_{\theta_{1}^{ \pm}} s_{\theta_{3}^{ \pm}} & -\left(c_{\theta_{1}^{ \pm}} s_{\theta_{3}^{ \pm}}+s_{\theta_{1}^{ \pm}} s_{\theta_{2}^{ \pm}} c_{\theta_{3}^{ \pm}}\right) & c_{\theta_{2}^{ \pm}} c_{\theta_{3}^{ \pm}}
\end{array}\right)
$$

where in the hybrid parametrization $\mathcal{P}_{I}$, these elements are given by,

$$
\begin{gathered}
\mathcal{C}_{11}=\frac{v_{1}}{v}, \quad \mathcal{C}_{12}=\frac{v_{2}}{v}, \quad \mathcal{C}_{13}=\sqrt{2} \frac{v_{t}}{v} \\
\mathcal{C}_{21}=\frac{x_{1}}{\sqrt{\mathcal{N}}}, \quad \mathcal{C}_{22}=\frac{x_{2}}{\sqrt{\mathcal{N}}}, \quad \mathcal{C}_{23}=\frac{1}{\sqrt{\mathcal{N}}} \\
\mathcal{C}_{31}=\mathcal{C}_{21}\left[m_{H_{1}^{ \pm}}^{2} \rightarrow m_{H_{2}^{ \pm}}^{2}\right], \quad \mathcal{C}_{32}=\mathcal{C}_{22}\left[m_{H_{1}^{ \pm}}^{2} \rightarrow m_{H_{2}^{ \pm}}^{2}\right], \quad \mathcal{C}_{33}=\mathcal{C}_{23}\left[m_{H_{1}^{ \pm}}^{2} \rightarrow m_{H_{2}^{ \pm}}^{2}\right]
\end{gathered}
$$

where $v_{0}=\sqrt{v_{1}^{2}+v_{2}^{2}}$ and

$$
\begin{gathered}
x_{1}=\frac{v_{0} c_{\beta}\left(v_{0}\left(\mathcal{M}_{12}^{ \pm}\left(\mathcal{M}_{13}^{ \pm} c_{\beta}+\mathcal{M}_{23}^{ \pm} s_{\beta}\right)+\mathcal{M}_{13}^{ \pm} m_{H_{1}^{ \pm}}^{2} s_{\beta}\right)+\sqrt{2} \mathcal{M}_{13}^{ \pm} \mathcal{M}_{23}^{ \pm} v_{t}\right)}{\sqrt{2} v_{0} v_{t}\left(m_{H_{1}^{ \pm}}^{2}\left(M_{23} c_{\beta}+\mathcal{M}_{13}^{ \pm} s_{\beta}\right)+\mathcal{M}_{12}^{ \pm}\left(\mathcal{M}_{13}^{ \pm} c_{\beta}+\mathcal{M}_{23}^{ \pm} s_{\beta}\right)\right)+v_{0}^{2} m_{H_{1}^{ \pm}}^{2}\left(c_{\beta} m_{H_{1}^{ \pm}}^{2} s_{\beta}+\mathcal{M}_{12}^{ \pm}\right)+2 \mathcal{M}_{13}^{ \pm} \mathcal{M}_{23}^{ \pm} v_{t}^{2}} \\
x_{2}=\frac{v_{0} s_{\beta}\left(\mathcal{M}_{23}^{ \pm}\left(v_{0} c_{\beta} m_{H_{1}^{ \pm}}^{2}+\sqrt{2} \mathcal{M}_{13}^{ \pm} v_{t}\right)+\mathcal{M}_{12}^{ \pm} v_{0}\left(\mathcal{M}_{13}^{ \pm} c_{\beta}+\mathcal{M}_{23}^{ \pm} s_{\beta}\right)\right)}{\sqrt{2} v_{0} v_{t}\left(m_{H_{1}^{ \pm}}^{2}\left(\mathcal{M}_{23}^{ \pm} c_{\beta}+\mathcal{M}_{13}^{ \pm} s_{\beta}\right)+\mathcal{M}_{12}^{ \pm}\left(\mathcal{M}_{13}^{ \pm} c_{\beta}+\mathcal{M}_{23}^{ \pm} s_{\beta}\right)\right)+v_{0}^{2} m_{H_{1}^{ \pm}}^{2}\left(c_{\beta} m_{H_{1}^{ \pm}}^{2} s_{\beta}+\mathcal{M}_{12}^{ \pm}\right)+2 \mathcal{M}_{13}^{ \pm} \mathcal{M}_{23}^{ \pm} v_{t}^{2}} \\
\mathcal{N}=\sqrt{1+x_{1}^{2}+x_{2}^{2}}
\end{gathered}
$$

Furthermore

$$
\tan \theta_{1}^{ \pm}=\frac{\mathcal{C}_{12}}{\mathcal{C}_{11}}=\frac{v_{2}}{v_{1}} ; \quad \tan \theta_{2}^{ \pm}=\frac{\mathcal{C}_{13}}{\mathcal{C}_{12}} s_{\theta_{1}^{ \pm}} ; \quad \tan \theta_{3}^{ \pm}=\frac{\mathcal{C}_{23}}{\mathcal{C}_{33}}
$$

\section{2. $\mathcal{C} \mathcal{P}_{\text {odd }}$ sector}

For the terms of odd sector, the rotation matrix $\mathcal{O}$ can be expressed in terms of the mixing angles $\beta_{i}$ as,

$$
\begin{aligned}
& \mathcal{O}=\left(\begin{array}{lll}
\mathcal{O}_{11} & \mathcal{O}_{12} & \mathcal{O}_{13} \\
\mathcal{O}_{21} & \mathcal{O}_{22} & \mathcal{O}_{23} \\
\mathcal{O}_{31} & \mathcal{O}_{32} & \mathcal{O}_{33}
\end{array}\right)=\left(\begin{array}{ccc}
c_{\beta_{1}} c_{\beta_{2}} & s_{\beta_{1}} c_{\beta_{2}} & s_{\beta_{2}} \\
-\left(c_{\beta_{1}} s_{\beta_{2}} s_{\beta_{3}}+s_{\theta_{1}^{ \pm}} c_{\beta_{3}}\right) & c_{\beta_{1}} c_{\beta_{3}}-s_{\beta_{1}} s_{\beta_{2}} s_{\beta_{3}} & c_{\beta_{2}} s_{\beta_{3}} \\
-c_{\beta_{1}} s_{\beta_{2}} c_{\beta_{3}}+s_{\beta_{1}} s_{\beta_{3}} & -\left(c_{\beta_{1}} s_{\beta_{3}}+s_{\beta_{1}} s_{\beta_{2}} c_{\beta_{3}}\right) & c_{\beta_{2}} c_{\beta_{3}}
\end{array}\right) \\
& \mathcal{O}_{11}=\frac{v_{1}}{v}, \quad \mathcal{O}_{12}=\frac{v_{2}}{v}, \quad \mathcal{O}_{13}=\sqrt{2} \frac{v_{t}}{v} \\
& \mathcal{O}_{21}=\frac{y_{1}}{\sqrt{\mathcal{N}_{1}}}, \quad \mathcal{O}_{22}=\frac{y_{2}}{\sqrt{\mathcal{N}_{1}}}, \quad \mathcal{O}_{23}=\frac{1}{\sqrt{\mathcal{N}_{1}}} \\
& \mathcal{O}_{31}=\mathcal{O}_{21}\left[m_{A_{1}}^{2} \rightarrow m_{A_{2}}^{2}\right], \quad \mathcal{O}_{32}=\mathcal{O}_{22}\left[m_{A_{1}}^{2} \rightarrow m_{A_{2}}^{2}\right], \quad \mathcal{O}_{33}=\mathcal{O}_{23}\left[m_{A_{1}}^{2} \rightarrow m_{A_{2}}^{2}\right]
\end{aligned}
$$

with $v_{0}=\sqrt{v_{1}^{2}+v_{2}^{2}}$ and, 


$$
\begin{aligned}
& y_{1}=\frac{v_{0} c_{\beta}\left(v_{0}\left(\mathcal{M}_{13}^{\text {odd }} m_{A_{1}}^{2} s_{\beta}+\mathcal{M}_{12}^{\text {odd }}\left(\mathcal{M}_{13}^{\text {odd }} c_{\beta}+\mathcal{M}_{23}^{\text {odd }} s_{\beta}\right)\right)+2 \mathcal{M}_{13}^{\text {odd }} \mathcal{M}_{23}^{\text {odd }} v_{t}\right)}{2 v_{0} v_{t}\left(m_{A_{1}}^{2}\left(\mathcal{M}_{23}^{\text {odd }} c_{\beta}+\mathcal{M}_{13}^{\text {odd }} s_{\beta}\right)+\mathcal{M}_{12}^{\text {odd }}\left(\mathcal{M}_{13}^{\text {odd }} c_{\beta}+\mathcal{M}_{23}^{\text {odd }} s_{\beta}\right)\right)+v_{0}^{2} m_{A_{1}}^{2}\left(m_{A_{1}}^{2} c_{\beta} s_{\beta}+\mathcal{M}_{12}^{\text {odd }}\right)+4 \mathcal{M}_{13}^{\text {odd }} \mathcal{M}_{23}^{\text {odd }} v_{t}^{2}} \\
& y_{2}=\frac{v_{0} s_{\beta}\left(\mathcal{M}_{23}^{\text {odd }}\left(v_{0} m_{A_{1}}^{2} c_{\beta}+2 \mathcal{M}_{13}^{\text {odd }} v_{t}\right)+\mathcal{M}_{12}^{\text {odd }} v_{0}\left(\mathcal{M}_{13}^{\text {odd }} c_{\beta}+\mathcal{M}_{23}^{\text {odd }} s_{\beta}\right)\right)}{2 v_{0} v_{t}\left(m_{A_{1}}^{2}\left(\mathcal{M}_{23}^{\text {odd }} c_{\beta}+\mathcal{M}_{13}^{\text {odd }} s_{\beta}\right)+\mathcal{M}_{12}^{\text {odd }}\left(\mathcal{M}_{13}^{\text {odd }} c_{\beta}+\mathcal{M}_{23}^{\text {odd }} s_{\beta}\right)\right)+v_{0}^{2} m_{A_{1}}^{2}\left(m_{A_{1}}^{2} c_{\beta} s_{\beta}+\mathcal{M}_{12}^{\text {odd }}\right)+4 \mathcal{M}_{13}^{\text {odd }} \mathcal{M}_{23}^{\text {odd }} v_{t}^{2}} \\
& \mathcal{N}_{1}=\sqrt{1+y_{1}^{2}+y_{2}^{2}}
\end{aligned}
$$

and

$$
\tan \beta_{1}=\frac{\mathcal{O}_{12}}{\mathcal{O}_{11}}=\frac{v_{2}}{v_{1}}, \quad \tan \beta_{2}=\frac{\mathcal{O}_{13}}{\mathcal{O}_{12}} s_{\beta_{1}}, \quad \tan \beta_{3}=\frac{\mathcal{O}_{23}}{\mathcal{O}_{33}}
$$

\section{APPENDIX B: SETTING THE MODEL PARAMETERS}

There are currently two choices of input parameters implemented in 2HDMcT. To ease export formalities for second set, a simplified approximations should be adopted for such purpose. Indeed, the $\mathcal{C}_{i j}(i, j=1,2,3)$ and $\mathcal{O}_{i j}(i, j=1,2,3)$ are nearly the same as $v_{t} \ll v_{0}$, which involves

$$
\begin{gathered}
\theta_{2}^{ \pm}=\beta_{2}=0 \\
\sin \theta_{1}^{ \pm}=\sin \beta_{1}=\frac{v_{2}}{v_{0}}=\sin \beta \\
\cos \theta_{1}^{ \pm}=\cos \beta_{1}=\frac{v_{1}}{v_{0}}=\cos \beta
\end{gathered}
$$

Using the Eqs. (B1)-(B3), the matrices $\mathcal{C}$ and $\mathcal{O}$ become,

$$
\mathcal{C}=\left(\begin{array}{ccc}
c_{\beta} & s_{\beta} & 0 \\
-s_{\beta} c_{\theta_{3}^{ \pm}} & c_{\beta} c_{\theta_{3}^{ \pm}} & s_{\theta_{3}^{ \pm}} \\
s_{\beta} s_{\theta_{3}^{ \pm}} & -c_{\beta} s_{\theta_{3}^{ \pm}} & c_{\theta_{3}^{ \pm}}
\end{array}\right) \quad, \quad \mathcal{O}=\left(\begin{array}{ccc}
c_{\beta} & s_{\beta} & 0 \\
-s_{\beta} c_{\beta_{3}} & c_{\beta} c_{\beta_{3}} & s_{\beta_{3}} \\
s_{\beta} s_{\beta_{3}} & -c_{\beta} s_{\beta_{3}} & c_{\beta_{3}}
\end{array}\right)
$$

using again Eqs. (16), (21), (26), (32), and (B4), the nonphysical parameters can be expressed through the masses, mixing angles, $\mu_{1}$ and $m_{12}^{2}$. The input set becomes:

$$
\mathcal{P}_{I I}=\left\{\mu_{1}, v_{t}, \alpha_{1,2,3}, \theta_{2}^{ \pm}, \beta_{3}, \tan \beta, m_{h_{1}, h_{2}, h_{3}}, m_{A_{1}, A_{2}}, m_{H_{1}^{ \pm}, H_{2}^{ \pm}}, m_{H^{ \pm \pm}}\right\} .
$$

One can then provide the following expressions for the nonphysical parameters,

$$
\begin{aligned}
\lambda_{1}= & \frac{\operatorname{sc}_{\beta}^{2}\left(-m_{A_{2}}^{2} s_{\beta} s_{\beta_{3}}^{2}\left(s_{\beta}+t_{\beta}\right)+2 c_{\alpha_{1}}^{2}\left(c_{\alpha_{2}}^{2} m_{h_{1}}^{2}+s_{\alpha_{2}}^{2}\left(c_{\alpha_{3}}^{2} m_{h_{3}}^{2}+m_{h_{2}}^{2} s_{\alpha_{3}}^{2}\right)\right)\right.}{2 v_{0}^{2}} \\
& \times \frac{\left.2 s_{\alpha_{1}}^{2}\left(c_{\alpha_{3}}^{2} m_{h_{2}}^{2}+m_{h_{3}}^{2} s_{\alpha_{3}}^{2}\right)+\left(m_{h_{2}}^{2}-m_{h_{3}}^{2}\right) s_{2 \alpha_{1}} s_{\alpha_{2}} s_{2 \alpha_{3}}-2 m_{12}^{2} t_{\beta}+2 \sqrt{2} \mu_{1} v_{t}\right)}{2 v_{0}^{2}} \\
\lambda_{2}= & \frac{1}{2 v_{0}^{2}}\left(\operatorname { c s } _ { \beta } ^ { 2 } \left(-m_{A_{2}}^{2} c_{\beta}\left(c_{\beta}+1\right) s_{\beta_{3}}^{2}+2 c_{\alpha_{1}}^{2}\left(c_{\alpha_{3}}^{2} m_{h_{2}}^{2}+m_{h_{3}}^{2} s_{\alpha_{3}}^{2}\right)+2 s_{\alpha_{1}}^{2}\left(c_{\alpha_{2}}^{2} m_{h_{1}}^{2}+s_{\alpha_{2}}^{2}\left(c_{\alpha_{3}}^{2} m_{h_{3}}^{2}+m_{h_{2}}^{2} s_{\alpha_{3}}^{2}\right)\right)\right.\right. \\
& \left.\left.-2 m_{12}^{2} \mathrm{ct}_{\beta}+2 \sqrt{2} \mu_{1} \mathrm{ct}_{\beta}^{2} v_{t}+\left(m_{h_{3}}^{2}-m_{h_{2}}^{2}\right) s_{2 \alpha_{1}} s_{\alpha_{2}} s_{2 \alpha_{3}}\right)\right)
\end{aligned}
$$




$$
\begin{aligned}
\lambda_{3}= & \frac{1}{2 v_{0}^{2}}\left(m_{A_{2}}^{2} s_{\beta_{3}}^{2}\left(\operatorname{sc}_{\beta}+1\right)+\operatorname{cs}_{\beta} \operatorname{sc}_{\beta}\left(c_{\alpha_{1}}^{2}\left(m_{h_{3}}^{2}-m_{h_{2}}^{2}\right) s_{\alpha_{2}} s_{2 \alpha_{3}}+s_{2 \alpha_{1}}\left(c_{\alpha_{2}}^{2} m_{h_{1}}^{2}+c_{\alpha_{3}}^{2}\left(m_{h_{3}}^{2} s_{\alpha_{2}}^{2}-m_{h_{2}}^{2}\right)\right)-2 m_{12}^{2}\right)+4 c_{\theta_{3}^{ \pm}}^{2} m_{h_{1}}^{2}\right. \\
& \left.+2 \operatorname{cs}_{2 \beta}\left(\left(m_{h_{2}}^{2}-m_{h_{3}}^{2}\right) s_{\alpha_{2}} s_{2 \alpha_{3}} s_{\alpha_{1}}^{2}+s_{2 \alpha_{1}} s_{\alpha_{3}}^{2}\left(m_{h_{2}}^{2} s_{\alpha_{2}}^{2}-m_{h_{3}}^{2}\right)\right)-2 \sqrt{2} \mu_{1} \operatorname{cs}_{\beta}^{2} v_{t}-4 m_{h_{2}}^{2} s_{\theta_{3}^{ \pm}}^{2} \mathrm{sc}_{\beta}\right) \\
\lambda_{4}= & \frac{2 m_{A_{1}}^{2} c_{\beta_{3}}^{2}-m_{A_{2}}^{2} s_{\beta_{3}}^{2}\left(\operatorname{sc}_{\beta}-1\right)-4 c_{\theta_{3}^{ \pm}}^{2} m_{h_{1}}^{2}+2 m_{12}^{2} \operatorname{cs}_{\beta} \operatorname{sc}_{\beta}-2 \sqrt{2} \mu_{1} \operatorname{cs}_{\beta}^{2} v_{t}+4 m_{h_{2}}^{2} s_{\theta_{3}^{ \pm}}^{2} \mathrm{sc}_{\beta}}{2 v_{0}^{2}}
\end{aligned}
$$$$
\lambda_{5}=\frac{-2 m_{A_{1}}^{2} c_{\beta_{3}}^{2}+m_{A_{2}}^{2} s_{\beta_{3}}^{2}\left(\operatorname{sc}_{\beta}-1\right)+2 m_{12}^{2} \operatorname{cs}_{\beta} \operatorname{sc}_{\beta}+2 \sqrt{2} \mu_{1} \mathrm{cs}_{\beta}^{2} v_{t}}{2 v_{0}^{2}}
$$$$
\lambda_{6}=\frac{\operatorname{sc}_{\beta}\left(8 v_{0} c_{\frac{\beta}{2}}^{4} s_{\frac{\beta}{2}}^{2}\left(m_{A_{2}}^{2} c_{2 \beta_{3}}-m_{A_{2}}^{2}-4 c_{2 \theta_{3}^{ \pm}} m_{h_{2}}^{2}+4 m_{h_{2}}^{2}\right)+c_{\alpha_{1}} s_{2 \alpha_{2}} v_{t}\left(c_{2 \alpha_{3}}\left(m_{h_{2}}^{2}-m_{h_{3}}^{2}\right)\right.\right.}{4 v_{0} v_{t}^{2}}
$$$$
\times \frac{\left.\left.2 m_{h_{1}}^{2}-m_{h_{2}}^{2}-m_{h_{3}}^{2}\right)+2 c_{\alpha_{2}}\left(m_{h_{3}}^{2}-m_{h_{2}}^{2}\right) s_{\alpha_{1}} s_{2 \alpha_{3}} v_{t}\right)}{4 v_{0} v_{t}^{2}}
$$$$
\lambda_{7}=\frac{2 v_{0} c_{\frac{\beta}{2}}^{2}\left(m_{A_{2}}^{2} c_{2 \beta_{3}}-m_{A_{2}}^{2}-4 c_{2 \theta_{3}^{ \pm}} m_{h_{2}}^{2}+4 m_{h_{2}}^{2}\right)+\operatorname{cs}_{\beta} v_{t}\left(s _ { \alpha _ { 1 } } s _ { 2 \alpha _ { 2 } } \left(c_{2 \alpha_{3}}\left(m_{h_{2}}^{2}-m_{h_{3}}^{2}\right)\right.\right.}{4 v_{0} v_{t}^{2}}
$$$$
\frac{\left.\left.2 m_{h_{1}}^{2}-m_{h_{2}}^{2}-m_{h_{3}}^{2}\right)+2 c_{\alpha_{1}} c_{\alpha_{2}}\left(m_{h_{2}}^{2}-m_{h_{3}}^{2}\right) s_{2 \alpha_{3}}\right)}{4 v_{0} v_{t}^{2}}
$$$$
\lambda_{8}=\frac{s_{\beta}\left(s_{\beta}+t_{\beta}\right)\left(m_{A_{2}}^{2} s_{\beta_{3}}^{2}-2 m_{h_{2}}^{2} s_{\theta_{3}^{ \pm}}^{2}\right)}{v_{t}^{2}}
$$$$
\lambda_{9}=\frac{c_{\frac{\beta}{2}}^{2}\left(m_{A_{2}}^{2}\left(-c_{2 \beta_{3}}\right)+m_{A_{2}}^{2}+2 c_{2 \theta_{3}^{ \pm}} m_{h_{2}}^{2}-2 m_{h_{2}}^{2}\right)}{v_{t}^{2}}
$$

$\bar{\lambda}_{8}=\frac{2 v_{0}^{2} c_{\frac{\beta}{2}}^{6} s_{\frac{\beta}{2}}^{2}\left(m_{A_{2}}^{2}\left(1-c_{2 \beta_{3}}\right)+\left(8 c_{2 \theta_{3}^{ \pm}}-8\right) m_{h_{2}}^{2}\right)+c_{\alpha_{2}}^{2} v_{t}^{2}\left(c_{\alpha_{3}}^{2} m_{h_{3}}^{2}+m_{h_{2}}^{2} s_{\alpha_{3}}^{2}\right)+v_{t}^{2}\left(m_{h_{1}}^{2} s_{\alpha_{2}}^{2}+2 m_{H^{\mp \pm}}^{2}\right)}{2 v_{t}^{4}}$

$\bar{\lambda}_{9}=\frac{2 v_{0}^{2} c_{\frac{\beta}{2}}^{6} s_{\frac{\beta}{2}}^{2}\left(m_{A_{2}}^{2} c_{2 \beta_{3}}-m_{A_{2}}^{2}-4 c_{2 \theta_{3}^{ \pm}} m_{h_{2}}^{2}+4 m_{h_{2}}^{2}\right)-m_{H^{ \pm \pm}}^{2} v_{t}^{2}}{v_{t}^{4}}$

$\mu_{2}=\frac{m_{A_{2}}^{2} s_{\beta}^{2} s_{\beta_{3}}^{2}}{2 \sqrt{2} v_{t}}+\mu_{1} \mathrm{ct}_{\beta}^{2}$

$\mu_{3}=\frac{m_{A_{2}}^{2}\left(c_{\beta}+1\right) s_{\beta} s_{\beta_{3}}^{2}-2 \sqrt{2} \mu_{1} \mathrm{ct}_{\beta} v_{t}}{\sqrt{2} v_{t}}$

with $\mathrm{t}_{x}=\tan x, \mathrm{ct}_{x}=1 / \tan x, \mathrm{c}_{x}=\cos x, \mathrm{~s}_{x}=\sin x, \mathrm{~s}_{2 x}=\sin 2 x, \mathrm{cs}_{x}=1 / \cos x, \mathrm{se}_{x}=1 / \sin x$

\section{APPENDIX C: UNITARITY CONSTRAINTS MATRICES}

The unitarity constraints are derived in the basis of unrotated states, corresponding to the fields before electroweak symmetry breaking. The quartic scalar vertices have in this case a much simpler form than the complicated functions of $\lambda_{i}, \alpha_{1}, \mathcal{O}_{i j}$, and $\mathcal{C}_{i j}$ obtained in the physical basis $\left(H^{ \pm \pm}, H_{1}^{ \pm}, H_{2}^{ \pm}, G^{ \pm}, H_{1}, H_{2}, H_{3}, A_{1}, A_{2}\right.$, and $\left.G^{0}\right)$ of 
mass eigenstate fields. The $S$-matrix for the physical fields is related by a unitary transformation to the $S$-matrix for the unrotated fields.

Close inspection shows that the full set of 2-body scalar scattering processes leads to a $40 \times 40 S$-matrix which can be decomposed into 7 block submatrices corresponding to mutually unmixed sets of channels with definite charge and $C P$ states. One has the following submatrix dimensions, structured in terms of net electric charge in the initial/final states: $S^{(1)}(18 \times 18), S^{(2)}(10 \times 10)$ and $S^{(3)}(3 \times 3)$, corresponding to 0 -charge channels, $S^{(4)}(21 \times 21)$ corresponding to the 1-charge channels, $S^{(5)}(12 \times 12)$ corresponding to the 2-charge channels, $S^{(6)}(3 \times 3)$ corresponding to the 3-charge channels and finally $S^{(7)}(1 \times 1)$ corresponding to the 4-charge channels.

The first submatrix $\mathcal{M}_{1}$ corresponds to scattering whose initial and final states are one of the following: $\left(\phi_{1}^{+} \delta^{-}\right.$, $\delta^{+} \phi_{1}^{-}, \phi_{2}^{+} \delta^{-}, \delta^{+} \phi_{2}^{-}, \phi_{1}^{+} \phi_{2}^{-}, \phi_{2}^{+} \phi_{1}^{-}, \rho_{1} \eta_{0}, \rho_{2} \eta_{0}, \rho_{1} \eta_{2}, \rho_{0} \eta_{1}$, $\left.\rho_{0} \eta_{2}, \rho_{2} \eta_{1}, \eta_{1} \eta_{0}, \eta_{2} \eta_{0}, \eta_{1} \eta_{2}, \rho_{1} \rho_{0}, \rho_{2} \rho_{0}, \rho_{1} \rho_{2}\right)$. With the help of Mathematica one finds,

$$
\mathcal{M}_{1}=\left(\begin{array}{c|c}
\mathcal{M}_{1}^{11}(7 \times 7) & \mathcal{M}_{1}^{12}(7 \times 11) \\
\hline \mathcal{M}_{1}^{21}(11 \times 7) & \mathcal{M}_{1}^{22}(11 \times 11)
\end{array}\right)
$$

with,

$$
\begin{aligned}
& \mathcal{M}_{1}^{11}(7 \times 7)=\left(\begin{array}{ccccccc}
\tilde{\lambda}_{68} & 0 & 0 & 0 & 0 & 0 & -i \frac{\lambda_{8}}{2 \sqrt{2}} \\
0 & \tilde{\lambda}_{68} & 0 & 0 & 0 & 0 & +i \frac{\lambda_{8}}{2 \sqrt{2}} \\
0 & 0 & \tilde{\lambda}_{79} & 0 & 0 & 0 & 0 \\
0 & 0 & 0 & \tilde{\lambda}_{79} & 0 & 0 & 0 \\
0 & 0 & 0 & 0 & \lambda_{34}^{+} & 2 \lambda_{5} & 0 \\
0 & 0 & 0 & 0 & 2 \lambda_{5} & \lambda_{34}^{+} & 0 \\
+i \frac{\lambda_{8}}{2 \sqrt{2}} & -i \frac{\lambda_{8}}{2 \sqrt{2}} & 0 & 0 & 0 & 0 & \lambda_{68}^{+}
\end{array}\right) \\
& \mathcal{M}_{1}^{22}(11 \times 11)=\left(\begin{array}{ccccccccccc}
\lambda_{79}^{+} & 0 & 0 & 0 & 0 & 0 & 0 & 0 & 0 & 0 & 0 \\
0 & \lambda_{L} & 0 & 0 & \lambda_{5} & 0 & 0 & 0 & 0 & 0 & 0 \\
0 & 0 & \lambda_{68}^{+} & 0 & 0 & 0 & 0 & 0 & 0 & 0 & 0 \\
0 & 0 & 0 & \lambda_{79}^{+} & 0 & 0 & 0 & 0 & 0 & 0 & 0 \\
0 & \lambda_{5} & 0 & 0 & \lambda_{L} & 0 & 0 & 0 & 0 & 0 & 0 \\
0 & 0 & 0 & 0 & 0 & \lambda_{68}^{+} & 0 & 0 & 0 & 0 & 0 \\
0 & 0 & 0 & 0 & 0 & 0 & \bar{\lambda}_{79}^{+} & 0 & 0 & 0 & 0 \\
0 & 0 & 0 & 0 & 0 & 0 & 0 & \lambda_{345} & 0 & 0 & \lambda_{5} \\
0 & 0 & 0 & 0 & 0 & 0 & 0 & 0 & \lambda_{68}^{+} & 0 & 0 \\
0 & 0 & 0 & 0 & 0 & 0 & 0 & 0 & 0 & \bar{\lambda}_{79}^{+} & 0 \\
0 & 0 & 0 & 0 & 0 & 0 & 0 & \lambda_{5} & 0 & 0 & \lambda_{345}
\end{array}\right) \\
& \mathcal{M}_{1}^{12}(7 \times 11)=\left(\begin{array}{ccccccccccc}
0 & 0 & +i \frac{\lambda_{8}}{2 \sqrt{2}} & 0 & 0 & \frac{\lambda_{8}}{2 \sqrt{2}} & 0 & 0 & \frac{\lambda_{8}}{2 \sqrt{2}} & 0 & 0 \\
0 & 0 & -i \frac{\lambda_{8}}{2 \sqrt{2}} & 0 & 0 & \frac{\lambda_{8}}{2 \sqrt{2}} & 0 & 0 & \frac{\lambda_{8}}{2 \sqrt{2}} & 0 & 0 \\
-i \frac{\lambda_{9}}{2 \sqrt{2}} & 0 & 0 & +i \frac{\lambda_{9}}{2 \sqrt{2}} & 0 & 0 & \frac{\lambda_{9}}{2 \sqrt{2}} & 0 & 0 & \frac{\lambda_{9}}{2 \sqrt{2}} & 0 \\
i \frac{\lambda_{9}}{2 \sqrt{2}} & 0 & 0 & -i \frac{\lambda_{9}}{2 \sqrt{2}} & 0 & 0 & \frac{\bar{\lambda}_{9}}{2 \sqrt{2}} & 0 & 0 & \frac{\bar{\lambda}_{9}}{2 \sqrt{2}} & 0 \\
0 & -i \frac{\lambda_{45}^{-}}{2} & 0 & 0 & +i \frac{\lambda_{45}^{-}}{2} & 0 & 0 & \frac{\lambda_{45}^{+}}{2} & 0 & 0 & \frac{\lambda_{45}^{+}}{2} \\
0 & +i \frac{\lambda_{45}^{-}}{2} & 0 & 0 & -i \frac{\lambda_{45}^{-}}{2} & 0 & 0 & \frac{\lambda_{45}^{+}}{2} & 0 & 0 & \frac{\lambda_{45}^{+}}{2} \\
0 & 0 & 0 & 0 & 0 & 0 & 0 & 0 & 0 & 0 & 0
\end{array}\right) \\
& \mathcal{M}_{1}^{12}(11 \times 7)=\left(\mathcal{M}_{1}^{12}(7 \times 11)\right)^{*}
\end{aligned}
$$

where $\lambda_{i j k}=\lambda_{i}+\lambda_{j}+\lambda_{k}, \tilde{\lambda}_{i j}=\lambda_{i}+\frac{\lambda_{j}}{2}, \lambda_{i j}^{ \pm}=\lambda_{i} \pm \lambda_{j}$, and $\lambda_{L}=\lambda_{3}+\lambda_{4}-\lambda_{5}$. 
The second submatrix $\mathcal{M}_{2}$ corresponds to scattering with one of the following initial and final states: $\left(\rho_{1} \eta_{1}, \rho_{2} \eta_{2}, \rho_{0} \eta_{0}\right)$. One finds that $\mathcal{M}_{3}$ given by:

$$
\mathcal{M}_{2}=\left(\begin{array}{ccc}
\lambda_{1} & \lambda_{5} & 0 \\
\lambda_{5} & \lambda_{2} & 0 \\
0 & 0 & 2 \bar{\lambda}_{89}^{+}
\end{array}\right)
$$

The third submatrix $\mathcal{M}_{3}$ corresponds to scattering with one of the following initial and final states: $\left(\phi_{1}^{+} \phi_{1}^{-}, \phi_{2}^{+} \phi_{2}^{-}, \delta^{+} \delta^{-}\right.$, $\left.\delta^{++} \delta^{--}, \frac{\eta_{1} \eta_{1}}{\sqrt{2}}, \frac{\eta_{2} \eta_{2}}{\sqrt{2}}, \frac{\eta_{0} \eta_{0}}{\sqrt{2}}, \frac{\rho_{1} \rho_{1}}{\sqrt{2}}, \frac{\rho_{2} \rho_{2}}{\sqrt{2}}, \frac{\rho_{0} \rho_{0}}{\sqrt{2}}\right)$, where the $\sqrt{2}$ accounts for identical particle statistics. One finds that $\mathcal{M}_{2}$ is given by:

$$
\mathcal{M}_{3}=\left(\begin{array}{cccccccccc}
2 \lambda_{1} & \lambda_{34}^{+} & \tilde{\lambda}_{68} & \lambda_{68}^{+} & \frac{\lambda_{1}}{\sqrt{2}} & \frac{\lambda_{3}}{\sqrt{2}} & \frac{\lambda_{6}}{\sqrt{2}} & \frac{\lambda_{1}}{\sqrt{2}} & \frac{\lambda_{3}}{\sqrt{2}} & \frac{\lambda_{6}}{\sqrt{2}} \\
\lambda_{34}^{+} & 2 \lambda_{2} & \tilde{\lambda}_{79} & \lambda_{79}^{+} & \frac{\lambda_{3}}{\sqrt{2}} & \frac{\lambda_{2}}{\sqrt{2}} & \frac{\lambda_{7}}{\sqrt{2}} & \frac{\lambda_{3}}{\sqrt{2}} & \frac{\lambda_{2}}{\sqrt{2}} & \frac{\lambda_{7}}{\sqrt{2}} \\
\tilde{\lambda}_{68} & \tilde{\lambda}_{79} & 4 \tilde{\lambda}_{89} & 2 \bar{\lambda}_{89} & \frac{\tilde{\lambda}_{68}}{\sqrt{2}} & \frac{\tilde{\lambda}_{79}}{\sqrt{2}} & \sqrt{2} \bar{\lambda}_{89} & \frac{\tilde{\lambda}_{68}}{\sqrt{2}} & \frac{\tilde{\lambda}_{79}}{\sqrt{2}} & \sqrt{2} \bar{\lambda}_{89} \\
\lambda_{68}^{+} & \lambda_{79}^{+} & 2 \bar{\lambda}_{89} & 4 \bar{\lambda}_{89}^{+} & \frac{\lambda_{6}}{\sqrt{2}} & \frac{\lambda_{7}}{\sqrt{2}} & \sqrt{2} \bar{\lambda}_{8} & \frac{\lambda_{6}}{\sqrt{2}} & \frac{\lambda_{7}}{\sqrt{2}} & \sqrt{2} \bar{\lambda}_{8} \\
\frac{\lambda_{1}}{\sqrt{2}} & \frac{\lambda_{3}}{\sqrt{2}} & \frac{\tilde{\lambda}_{68}}{\sqrt{2}} & \frac{\lambda_{6}}{\sqrt{2}} & \frac{3 \lambda_{1}}{2} & \frac{\lambda_{345}}{2} & \frac{\lambda_{68}^{+}}{2} & \frac{\lambda_{1}}{2} & \frac{\lambda_{L}}{2} & \frac{\lambda_{68}^{+}}{2} \\
\frac{\lambda_{3}}{\sqrt{2}} & \frac{\lambda_{2}}{\sqrt{2}} & \frac{\tilde{7}_{99}}{\sqrt{2}} & \frac{\lambda_{7}}{\sqrt{2}} & \frac{\lambda_{345}}{2} & \frac{3 \lambda_{2}}{2} & \frac{\lambda_{79}^{+}}{2} & \frac{\lambda_{L}}{2} & \frac{\lambda_{2}}{2} & \frac{\lambda_{79}^{+}}{2} \\
\frac{\lambda_{6}}{\sqrt{2}} & \frac{\lambda_{7}}{\sqrt{2}} & \sqrt{2} \bar{\lambda}_{89}^{+} & \sqrt{2} \bar{\lambda}_{8} & \frac{\lambda_{68}^{+}}{2} & \frac{\lambda_{79}^{+}}{2} & 3 \bar{\lambda}_{89}^{+} & \frac{\lambda_{68}^{+}}{2} & \frac{\lambda_{79}^{+}}{2} & \bar{\lambda}_{89}^{+} \\
\frac{\lambda_{1}}{\sqrt{2}} & \frac{\lambda_{3}}{\sqrt{2}} & \frac{\tilde{x}_{68}}{\sqrt{2}} & \frac{\lambda_{6}}{\sqrt{2}} & \frac{\lambda_{1}}{2} & \frac{\lambda_{L}}{2} & \frac{\lambda_{68}^{+}}{2} & \frac{3 \lambda_{1}}{2} & \frac{\lambda_{345}}{2} & \frac{\lambda_{68}^{+}}{2} \\
\frac{\lambda_{3}}{\sqrt{2}} & \frac{\lambda_{2}}{\sqrt{2}} & \frac{\tilde{\lambda}_{79}}{\sqrt{2}} & \frac{\lambda_{7}}{\sqrt{2}} & \frac{\lambda_{L}}{2} & \frac{\lambda_{2}}{2} & \frac{\lambda_{79}^{+}}{2} & \frac{\lambda_{345}}{2} & \frac{3 \lambda_{2}}{2} & \frac{\lambda_{79}^{+}}{2} \\
\frac{\lambda_{6}}{\sqrt{2}} & \frac{\lambda_{7}}{\sqrt{2}} & \sqrt{2} \bar{\lambda}_{89}^{+} & \sqrt{2} \bar{\lambda}_{8} & \frac{\lambda_{68}^{+}}{2} & \frac{\lambda_{79}^{+}}{2} & \bar{\lambda}_{89}^{+} & \frac{\lambda_{68}^{+}}{2} & \frac{\lambda_{79}^{+}}{2} & 3 \bar{\lambda}_{89}^{+}
\end{array}\right)
$$

despite its apparently complicated structure, six eigenvalues for $\mathcal{M}_{3}$ come from a very long two polynomial equations of order 3. In order to solve these matrices, we use the Jacobi method.

The fourth submatrix $\mathcal{M}_{4}$ corresponds to scattering with initial and final states being one of the following 21 sates: $\left(\rho_{0} \phi_{1}^{+}, \rho_{1} \phi_{1}^{+}, \rho_{2} \phi_{1}^{+}, \eta_{0} \phi_{1}^{+}, \eta_{1} \phi_{1}^{+}, \eta_{2} \phi_{1}^{+}, \rho_{0} \phi_{2}^{+}, \rho_{1} \phi_{2}^{+}, \rho_{2} \phi_{2}^{+}, \eta_{0} \phi_{2}^{+}, \eta_{1} \phi_{2}^{+}, \eta_{2} \phi_{2}^{+}, \rho_{0} \delta^{+}, \rho_{1} \delta^{+}, \rho_{2} \delta^{+}, \eta_{0} \delta^{+}, \eta_{1} \delta^{+}, \eta_{2} \delta^{+}, \delta^{++} \delta^{-}\right.$, $\left.\delta^{++} \phi_{1}^{-}, \delta^{++} \phi_{2}^{-}\right)$. It reads that:

$$
\mathcal{M}_{4}=\left(\begin{array}{c|c|c}
\mathcal{M}_{4}^{11}(7 \times 7) & \mathcal{M}_{4}^{12}(7 \times 11) & \mathcal{M}_{4}^{13}(7 \times 11) \\
\hline \mathcal{M}_{4}^{21}(7 \times 7) & \mathcal{M}_{4}^{22}(7 \times 11) & \mathcal{M}_{4}^{23}(7 \times 11) \\
\hline \mathcal{M}_{4}^{31}(7 \times 7) & \mathcal{M}_{4}^{32}(7 \times 11) & \mathcal{M}_{4}^{33}(7 \times 11)
\end{array}\right)
$$

with, 
$\mathcal{M}_{4}^{11}(7 \times 7)=\left(\begin{array}{ccccccc}\lambda_{6} & 0 & 0 & 0 & 0 & 0 & 0 \\ 0 & \lambda_{1} & 0 & 0 & 0 & 0 & 0 \\ 0 & 0 & \lambda_{3} & 0 & 0 & 0 & 0 \\ 0 & 0 & 0 & \lambda_{6} & 0 & 0 & 0 \\ 0 & 0 & 0 & 0 & \lambda_{1} & 0 & 0 \\ 0 & 0 & 0 & 0 & 0 & \lambda_{3} & 0 \\ 0 & 0 & 0 & 0 & 0 & 0 & \lambda_{7}\end{array}\right), \quad \mathcal{M}_{4}^{22}(7 \times 7)=\left(\begin{array}{ccccccc}\lambda_{3} & 0 & 0 & 0 & 0 & 0 & 0 \\ 0 & \lambda_{2} & 0 & 0 & 0 & \frac{\lambda_{9}}{2 \sqrt{2}} & 0 \\ 0 & 0 & \lambda_{7} & 0 & 0 & 0 & 0 \\ 0 & 0 & 0 & \lambda_{3} & 0 & 0 & 0 \\ 0 & 0 & 0 & 0 & \lambda_{2} & i \frac{\lambda_{9}}{2 \sqrt{2}} & 0 \\ 0 & \frac{\lambda_{9}}{2 \sqrt{2}} & 0 & 0 & -i \frac{\lambda_{9}}{2 \sqrt{2}} & 2 \bar{\lambda}_{89}^{+} & 0 \\ 0 & 0 & 0 & 0 & 0 & 0 & \tilde{\lambda}_{68}\end{array}\right)$

$\mathcal{M}_{4}^{33}(7 \times 7)=\left(\begin{array}{ccccccc}\tilde{\lambda}_{79} & 0 & 0 & 0 & 0 & 0 & -\frac{\lambda_{9}}{2} \\ 0 & 2 \bar{\lambda}_{89}^{+} & 0 & 0 & -i \sqrt{2} \bar{\lambda}_{9} & 0 & 0 \\ 0 & 0 & \tilde{\lambda}_{68} & 0 & 0 & -i \frac{\lambda_{8}}{2} & 0 \\ 0 & 0 & 0 & \tilde{\lambda}_{79} & 0 & 0 & -i \frac{\lambda_{9}}{2} \\ 0 & i \sqrt{2} \bar{\lambda}_{9} & 0 & 0 & 2 \bar{\lambda}_{89}^{+} & 0 & 0 \\ 0 & 0 & i \frac{\lambda_{8}}{2} & 0 & 0 & \lambda_{68}^{+} & 0 \\ -\frac{\lambda_{9}}{2} & 0 & 0 & i \frac{\lambda_{9}}{2} & 0 & 0 & \lambda_{79}^{+}\end{array}\right)$

and

$$
\begin{aligned}
& \mathcal{M}_{4}^{12}(7 \times 7)=\left(\begin{array}{ccccccc}
0 & 0 & 0 & 0 & 0 & 0 & \frac{\lambda_{8}}{2 \sqrt{2}} \\
0 & \frac{\lambda_{45}^{+}}{2} & 0 & 0 & i \frac{\lambda_{54}^{-}}{2} & \frac{\lambda_{8}}{2 \sqrt{2}} & 0 \\
\frac{\lambda_{45}^{+}}{2} & 0 & 0 & i \frac{\lambda_{45}^{-}}{2} & 0 & 0 & 0 \\
0 & 0 & 0 & 0 & 0 & 0 & -i \frac{\lambda_{8}}{2 \sqrt{2}} \\
0 & i \frac{\lambda_{45}^{-}}{2} & 0 & 0 & \frac{\lambda_{45}^{+}}{2} & i \frac{\lambda_{8}}{2 \sqrt{2}} & 0 \\
i \frac{\lambda_{54}^{-}}{2} & 0 & 0 & \frac{\lambda_{45}^{+}}{2} & 0 & 0 & 0 \\
0 & 0 & 0 & 0 & 0 & 0 & 0
\end{array}\right), \quad \mathcal{M}_{4}^{13}(7 \times 7)=\left(\begin{array}{ccccccc}
0 & 0 & i \frac{\lambda_{8}}{2 \sqrt{2}} & 0 & 0 & 0 & 0 \\
0 & -i \frac{\lambda_{8}}{2 \sqrt{2}} & 0 & 0 & -\frac{\lambda_{8}}{2} & 0 & 0 \\
0 & 0 & 0 & 0 & 0 & 0 & 0 \\
0 & 0 & \frac{\lambda_{8}}{2 \sqrt{2}} & 0 & 0 & 0 & 0 \\
0 & \frac{\lambda_{8}}{2 \sqrt{2}} & 0 & 0 & -i \frac{\lambda_{8}}{2} & 0 & 0 \\
0 & 0 & 0 & 0 & 0 & 0 & 0 \\
\frac{\lambda_{9}}{2 \sqrt{2}} & 0 & 0 & i \frac{\lambda_{9}}{2 \sqrt{2}} & 0 & 0 & 0
\end{array}\right) \\
& \mathcal{M}_{4}^{23}(7 \times 7)=\left(\begin{array}{ccccccc}
0 & 0 & 0 & 0 & 0 & 0 & 0 \\
0 & -i \frac{\lambda_{9}}{2 \sqrt{2}} & 0 & 0 & -\frac{\lambda_{9}}{2} & 0 & 0 \\
-i \frac{\lambda_{9}}{2 \sqrt{2}} & 0 & 0 & \frac{\lambda_{9}}{2 \sqrt{2}} & 0 & 0 & 0 \\
0 & 0 & 0 & 0 & 0 & 0 & 0 \\
0 & \frac{\bar{\lambda}_{9}}{2 \sqrt{2}} & 0 & 0 & -i \frac{\bar{\lambda}_{9}}{2} & 0 & 0 \\
0 & 0 & 0 & 0 & -\sqrt{2} \bar{\lambda}_{9} & 0 & 0 \\
0 & 0 & 0 & 0 & 0 & -\frac{\lambda_{8}}{2} & 0
\end{array}\right)
\end{aligned}
$$

while the other off-diagonal lower elements in Eq. (C5) are related to upper off-diagonal ones by switching $i \leftrightarrow j$ in $\mathcal{M}_{4}^{i j}(7 \times 7)=\left(\mathcal{M}_{4}^{j i}(7 \times 7)\right)^{*}$ for $i>1 \wedge i>j \wedge i \neq j$. Solving the cubic polynomial equation given in Eq. (41), we find:

$$
\begin{aligned}
& a_{17}=\frac{1}{3}\left(b+2 \sqrt{p} \cos \left(\frac{\Lambda}{3}\right)\right) \\
& a_{18}=\frac{1}{3}\left(b+2 \sqrt{p} \cos \left(\frac{\Lambda+2 \pi}{3}\right)\right) \\
& a_{19}=\frac{1}{3}\left(b+2 \sqrt{p} \cos \left(\frac{\Lambda-2 \pi}{3}\right)\right)
\end{aligned}
$$


with

$$
\begin{aligned}
b & =2 \bar{\lambda}_{8}+\bar{\lambda}_{9}+\lambda_{1}+\lambda_{2} \\
p & =b^{2}-3 r \\
\Lambda & =\cos ^{-1}\left(\frac{q}{2 \sqrt{p^{3}}}\right)
\end{aligned}
$$

where $r, q$ stand for

$$
\begin{gathered}
r=2 \bar{\lambda}_{8} \lambda_{1}+\bar{\lambda}_{9} \lambda_{1}+2 \bar{\lambda}_{8} \lambda_{2}+\bar{\lambda}_{9} \lambda_{2}+\lambda_{1} \lambda_{2}-\lambda_{4}^{2}-\lambda_{8}^{2}-\lambda_{9}^{2} \\
q=2 b^{3}-9 b r-27 r_{1}
\end{gathered}
$$

and $r_{1}=-2 \bar{\lambda}_{8} \lambda_{1} \lambda_{2}-\bar{\lambda}_{9} \lambda_{1} \lambda_{2}+2 \bar{\lambda}_{8} \lambda_{4}^{2}+\bar{\lambda}_{9} \lambda_{4}^{2}+\lambda_{2} \lambda_{8}^{2}-2 \lambda_{4} \lambda_{8} \lambda_{9}+\lambda_{1} \lambda_{9}^{2}$

The fifth submatrix $\mathcal{M}_{5}$ corresponds to scattering with initial and final states being one of the following 12 states: $\left(\frac{\phi_{1}^{+} \phi_{1}^{+}}{\sqrt{2}}, \frac{\phi_{2}^{+} \phi_{2}^{+}}{\sqrt{2}}, \frac{\delta^{+} \delta^{+}}{\sqrt{2}}, \phi_{1}^{+} \phi_{2}^{+}, \phi_{1}^{+} \delta^{+}, \phi_{2}^{+} \delta^{+}, \delta^{++} \rho_{0}, \delta^{++} \rho_{1}, \delta^{++} \rho_{2}, \delta^{++} \eta_{0}, \delta^{++} \eta_{1}, \delta^{++} \eta_{2}\right)$. It reads,

$$
\mathcal{M}_{5}=\left(\begin{array}{cccccccccccc}
\lambda_{1} & \lambda_{5} & 0 & 0 & 0 & 0 & 0 & 0 & 0 & 0 & 0 & 0 \\
\lambda_{5} & \lambda_{2} & 0 & 0 & 0 & 0 & 0 & 0 & 0 & 0 & 0 & 0 \\
0 & 0 & 2 \tilde{\bar{\lambda}}_{89} & 0 & 0 & 0 & 0 & 0 & 0 & 0 & 0 & 0 \\
0 & 0 & 0 & \lambda_{34}^{+} & 0 & 0 & 0 & 0 & 0 & 0 & 0 & 0 \\
0 & 0 & 0 & 0 & \tilde{\lambda}_{68} & 0 & 0 & -\frac{\lambda_{8}}{2} & 0 & 0 & -i \frac{\lambda_{8}}{2} & 0 \\
0 & 0 & 0 & 0 & 0 & \tilde{\lambda}_{79} & 0 & 0 & -\frac{\lambda_{9}}{2} & 0 & 0 & -i \frac{\lambda_{9}}{2} \\
0 & 0 & 0 & 0 & 0 & 0 & 2 \bar{\lambda}_{8} & 0 & 0 & 0 & 0 & 0 \\
0 & 0 & 0 & 0 & -\frac{\lambda_{8}}{2} & 0 & 0 & \lambda_{6} & 0 & 0 & 0 & 0 \\
0 & 0 & 0 & 0 & 0 & -\frac{\lambda_{9}}{2} & 0 & 0 & \lambda_{7} & 0 & 0 & 0 \\
0 & 0 & 0 & 0 & 0 & 0 & 0 & 0 & 0 & 2 \bar{\lambda}_{8} & 0 & 0 \\
0 & 0 & 0 & 0 & i \frac{\lambda_{8}}{2} & 0 & 0 & 0 & 0 & 0 & \lambda_{6} & 0 \\
0 & 0 & 0 & 0 & 0 & i \frac{\lambda_{9}}{2} & 0 & 0 & 0 & 0 & 0 & \lambda_{7}
\end{array}\right)
$$

There are also triply charged states. The submatrix $\mathcal{M}_{6}$ corresponding to this case generates the scattering with initial and final states being one of the following $\left(\delta^{++} \phi_{1}^{+}\right.$, $\left.\delta^{++} \phi_{2}^{+}, \delta^{++} \delta^{+}\right)$, and is given by,

$$
\mathcal{M}_{6}=\left(\begin{array}{ccc}
\lambda_{68}^{+} & 0 & 0 \\
0 & \lambda_{79}^{+} & 0 \\
0 & 0 & 2 \bar{\lambda}_{89}
\end{array}\right)
$$

and finally, it is easy to check that there is just one quadruply charged state $\frac{1}{\sqrt{2}} \delta^{++} \delta^{++}$, leading to $\mathcal{M}_{7}=4 \bar{\lambda}_{89}^{+}$.

\section{APPENDIX D: BOUNDEDNESS FROM BELOW CONSTRAINTS}

To proceed to the most general case, we adopt a different parametrization of the fields that will turn out to be particularly convenient to entirely solve the problem. For that we combine both parametrizations used in $[15,57]$ and define:

$$
\begin{aligned}
r & \equiv \sqrt{H_{1}^{\dagger} H_{1}+H_{2}^{\dagger} H_{2}+\operatorname{Tr} \Delta^{\dagger} \Delta} \\
H_{1}^{\dagger} H_{1} & \equiv r^{2} \cos ^{2} \theta \sin ^{2} \phi \\
H_{2}^{\dagger} H_{2} & \equiv r^{2} \sin ^{2} \theta \sin ^{2} \phi
\end{aligned}
$$




$$
\begin{gathered}
\operatorname{Tr} \Delta^{\dagger} \Delta \equiv r^{2} \cos ^{2} \phi \\
\operatorname{Tr}\left(\Delta^{\dagger} \Delta\right)^{2} /\left(\operatorname{Tr} \Delta^{\dagger} \Delta\right)^{2} \equiv \epsilon \\
\left(H_{1}^{\dagger} \Delta \Delta^{\dagger} H_{1}\right) /\left(H_{1}^{\dagger} H_{1} \operatorname{Tr} \Delta^{\dagger} \Delta\right) \equiv \eta \\
\left(H_{2}^{\dagger} \Delta \Delta^{\dagger} H_{2}\right) /\left(H_{2}^{\dagger} H_{2} \operatorname{Tr} \Delta^{\dagger} \Delta\right) \equiv \zeta
\end{gathered}
$$

Obviously, when $H_{1}, H_{2}$ and $\Delta$ scan all the field space, the radius $r$ scans the domain $[0, \infty$, the angle $\theta \in[0,2 \pi]$, and the angle $\phi \in\left[0, \frac{\pi}{2}\right]$. Moreover, as $\frac{H_{1}^{\dagger} \cdot H_{2}}{\left|H_{1}\right|\left|H_{2}\right|}$ is a product of unit spinor, it is a complex number $\alpha+i \beta$ such that $|\alpha+i \beta| \leq 1$. We can rewrite it in polar coordinates as $\alpha+i \beta=\xi e^{i \psi r}$ with $\xi \in(0,1)$. We can also show that $\eta \in(0,1), \zeta \in(0,1)$, and $\epsilon \in\left[\frac{1}{2}, 1\right]$.

With this parametrization, one can cast $V^{(4)}\left(H_{1}, H_{2}, \Delta\right)$ in the following simple form,

$$
\begin{aligned}
V^{(4)}( & \left.r, c_{\theta}^{2}, s_{\phi}^{2}, c_{2 \psi}, \xi, \epsilon, \eta, \zeta\right) \\
= & r^{4}\left\{\lambda_{1} c_{\theta}^{4} s_{\phi}^{4}+\lambda_{2} s_{\theta}^{4} s_{\phi}^{4}+\lambda_{3} c_{\theta}^{2} s_{\theta}^{2} s_{\phi}^{4}+\lambda_{4} c_{\theta}^{2} s_{\theta}^{2} s_{\phi}^{4} \xi^{2}\right. \\
& +\lambda_{5} c_{\theta}^{2} s_{\theta}^{2} s_{\phi}^{4} \xi^{2} \cos 2 \psi+c_{\phi}^{4}\left(\bar{\lambda}_{8}+\epsilon \bar{\lambda}_{9}\right) \\
& \left.+c_{\theta}^{2} c_{\phi}^{2} s_{\phi}^{2}\left(\lambda_{6}+\eta \lambda_{8}\right)+s_{\theta}^{2} c_{\phi}^{2} s_{\phi}^{2}\left(\lambda_{7}+\zeta \lambda_{9}\right)\right\}
\end{aligned}
$$

To simplify we take:

$$
\begin{aligned}
& x \equiv \cos ^{2} \theta \\
& y \equiv \sin ^{2} \phi \\
& z \equiv \cos 2 \psi \in(-1,1)
\end{aligned}
$$

which allows us to rewrite the potential in the final form:

$$
\begin{aligned}
V^{(4)} / r^{4}= & \left\{\frac{\lambda_{1}}{2} x^{2}+\frac{\lambda_{2}}{2}(1-x)^{2}+\lambda_{3} x(1-x)\right. \\
& \left.+\lambda_{4} x(1-x) \xi^{2}+\lambda_{5} x(1-x) \xi^{2} z\right\} y^{2} \\
& +\left\{\bar{\lambda}_{8}+\epsilon \bar{\lambda}_{9}\right\}(1-y)^{2} \\
& +\left\{\left(\lambda_{6}+\eta \lambda_{8}\right) x+\left(\lambda_{7}+\zeta \lambda_{9}\right)(1-x)\right\} y(1-y)
\end{aligned}
$$

it is easy to find the constraint conditions by studying $V^{(4)}(x, y, z, \xi, \epsilon, \eta, \zeta)$ as a quadratic function using the fact that:

$$
\begin{aligned}
& f(y)=a y^{2}+b(1-y)^{2}+c y(1-y), \\
& y \in(0,1) \Leftrightarrow a>0, b>0 \text { and } c+2 \sqrt{a b}>0
\end{aligned}
$$

we can deduce the set of constraints as:

$$
\begin{aligned}
F_{I}(\xi, z) \equiv & \frac{\lambda_{1}}{2} x^{2}+\frac{\lambda_{2}}{2}(1-x)^{2}+\lambda_{3} x(1-x)+\lambda_{4} x(1-x) \xi^{2} \\
& +\lambda_{5} x(1-x) \xi^{2} z>0 \\
F_{I I}(\epsilon) \equiv & \bar{\lambda}_{8}+\epsilon \bar{\lambda}_{9}>0 \\
F_{I I I}(\eta, \zeta) \equiv & \left(\lambda_{6}+\eta \lambda_{8}\right) x+\left(\lambda_{7}+\zeta \lambda_{9}\right)(1-x) \\
> & -2 \sqrt{F_{I}(\xi, z) F_{I I}(\epsilon)}
\end{aligned}
$$

For $F_{I}(\xi, z)>0$ one can use Eq. (D13) again to get the ordinary $2 \mathrm{HDM} \mathrm{BFB}$ constraints taking into account if $\xi=0 ; 1$ and $z=-1 ; 1$ :

$$
\begin{aligned}
\lambda_{1}, \lambda_{2} & >0 \\
\lambda_{3}+\sqrt{\lambda_{1} \lambda_{2}} & >0 \\
\lambda_{3}+\lambda_{4}-\left|\lambda_{5}\right|+\sqrt{\lambda_{1} \lambda_{2}} & >0
\end{aligned}
$$

For $F_{I I}(\epsilon)$ which is monotonic function, the condition $0<F_{I I}(\epsilon)$ is s equivalent to $0<F_{I I}\left(\frac{1}{2}\right)$ and $0<F_{I I}(1)$. So that Eq. (D15) becomes,

$$
\bar{\lambda}_{8}+\bar{\lambda}_{9}>0, \quad \bar{\lambda}_{8}+\frac{1}{2} \bar{\lambda}_{9}>0
$$

For Eq. (D16), one can write it as:

$$
\begin{aligned}
& F_{I I I}(\eta, \zeta)+2 \sqrt{F_{I}(\xi, z) F_{I I}(\epsilon)}>0 \\
& \Leftrightarrow\left\{\begin{array}{l}
F_{I I I}(\eta, \zeta)>0 \text { and } F_{I}(\xi, z) F_{I I}(\epsilon)>0 \text { (i) } \\
\text { or } \\
F_{I I I}(\eta, \zeta) \leq 0 \text { and } 4 F_{I}(\xi, z) F_{I I}(\epsilon)>F_{I I I}^{2}(\eta, \zeta) \text { (ii) }
\end{array}\right.
\end{aligned}
$$

(i) scenario (i): starting with the fact that $x=\cos ^{2} \theta>0$ and $1-x=\sin ^{2} \theta>0$, thus $F_{I I I}(\eta, \zeta)>0 \Rightarrow$ generic relations:

$$
\begin{aligned}
& \lambda_{6}+\eta \lambda_{8}>0, \quad \forall \eta \in[0,1] \\
& \lambda_{7}+\zeta \lambda_{9}>0, \quad \forall \zeta \in[0,1]
\end{aligned}
$$

We note here that $\eta$ and $\zeta$ are independent parameters since there are no quartic couplings linking together $H_{1}, H_{2}$, and $\Delta$. Consequently the two monotonic function in (D22) and (D22) leads as above to,

$$
\begin{array}{ll}
\lambda_{6}>0, & \lambda_{6}+\lambda_{8}>0, \\
\lambda_{7}>0, & \lambda_{7}+\lambda_{9}>0
\end{array}
$$


(ii) scenario (ii): this scenario imply both $\left(\lambda_{6}+\eta \lambda_{8}\right)$ and $\left(\lambda_{7}+\zeta \lambda_{9}\right) \leq 0$ and leads to:

$$
\begin{aligned}
& \left\{2 \lambda_{1}\left(\bar{\lambda}_{8}+\epsilon \bar{\lambda}_{9}\right)-\left(\lambda_{6}+\eta \lambda_{8}\right)^{2}\right\} x^{2}+\left\{2 \lambda_{2}\left(\bar{\lambda}_{8}+\epsilon \bar{\lambda}_{9}\right)-\left(\lambda_{7}+\zeta \lambda_{9}\right)^{2}\right\}(1-x)^{2} \\
& \quad+\left\{4\left(\lambda_{3}+\lambda_{4} \xi^{2}+\lambda_{5} \xi^{2} z\right)\left(\bar{\lambda}_{8}+\epsilon \bar{\lambda}_{9}\right)-2\left(\lambda_{6}+\eta \lambda_{8}\right)\left(\lambda_{7}+\zeta \lambda_{9}\right)\right\} x(1-x)>0
\end{aligned}
$$

Applying the lemma given by Eq. (D13), we get the following generic new constraints,

$$
\begin{aligned}
& \lambda_{6}+\eta \lambda_{8}>-\sqrt{2 \lambda_{1}\left(\bar{\lambda}_{8}+\epsilon \bar{\lambda}_{9}\right)} \\
& \lambda_{7}+\zeta \lambda_{9}>-\sqrt{2 \lambda_{2}\left(\bar{\lambda}_{8}+\epsilon \bar{\lambda}_{9}\right)} \\
& 4\left(\lambda_{3}+\lambda_{4} \xi^{2}+\lambda_{5} \xi^{2} z\right)\left(\bar{\lambda}_{8}+\epsilon \bar{\lambda}_{9}\right)-2\left(\lambda_{6}+\eta \lambda_{8}\right)\left(\lambda_{7}+\zeta \lambda_{9}\right) \\
& \quad>-2 \sqrt{\left(2 \lambda_{1}\left(\bar{\lambda}_{8}+\epsilon \bar{\lambda}_{9}\right)-\left(\lambda_{6}+\eta \lambda_{8}\right)^{2}\right)\left(2 \lambda_{2}\left(\bar{\lambda}_{8}+\epsilon \bar{\lambda}_{9}\right)-\left(\lambda_{7}+\zeta \lambda_{9}\right)^{2}\right)}
\end{aligned}
$$

To continue, some major adjustments are needed to complete the set of BFB constraints. Firstly, we note further that each of two parameters $(\eta, \epsilon)$ and $(\zeta, \epsilon)$ cannot be anywhere in their small ranges bounded by the vertices $\left(0, \frac{1}{2}\right)$ and $(1,1)$ each. Indeed, from Eqs. [(D5)-(D6)] and [(D5)-(D7)], it can be shown that the possible values of $(\eta$, $\epsilon)$ and $(\zeta, \epsilon)$ correspond respectively to:

$$
\begin{aligned}
& 2 \eta^{2}-2 \eta+1 \leq \epsilon \leq 1, \\
& 2 \zeta^{2}-2 \zeta+1 \leq \epsilon \leq 1
\end{aligned}
$$

which define the shaded region depicted in Fig. 12.

Returning to the Eqs. (D26) and (D27), they may be formulated as,

$$
\begin{aligned}
& 0<\lambda_{6}+\eta \lambda_{8}+\sqrt{2 \lambda_{1}\left(\bar{\lambda}_{8}+\epsilon \bar{\lambda}_{9}\right)} \equiv G_{I}(\eta, \epsilon) \\
& 0<\lambda_{7}+\zeta \lambda_{9}+\sqrt{2 \lambda_{2}\left(\bar{\lambda}_{8}+\epsilon \bar{\lambda}_{9}\right)} \equiv G_{I I}(\zeta, \epsilon)
\end{aligned}
$$

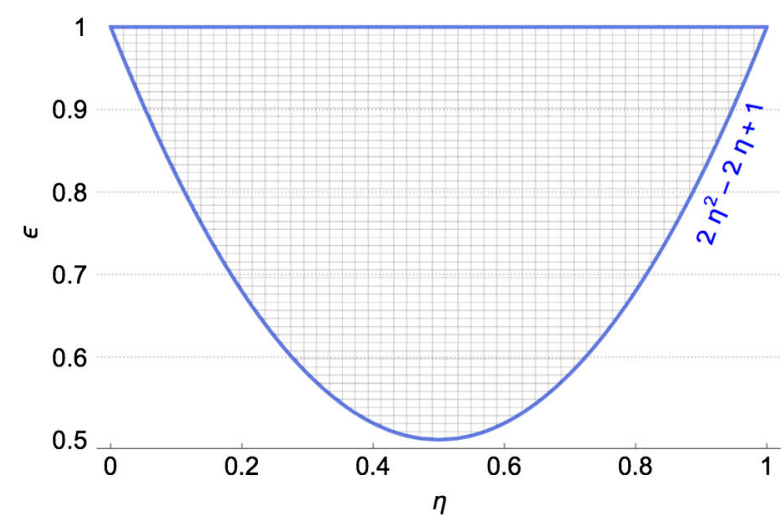

and see how to get rid of $\eta, \zeta$ and $\epsilon$. The minimum of $G_{I}(\eta, \epsilon)$ and $G_{I I}(\zeta, \epsilon)$ occurs at the border of the shaded regions in Fig. 12 since both functions are monotonic in both $(\eta, \epsilon)$ and $(\zeta, \epsilon)$, respectively. Otherwise, $0<G_{I}(\eta, \epsilon) \Leftrightarrow 0<\min G_{I}(\eta, \epsilon) \forall \eta, \epsilon$, and by the same way $0<G_{I I}(\zeta, \epsilon) \Leftrightarrow 0<\min G_{I I}(\zeta, \epsilon) \forall \zeta, \epsilon$. Such minimums that are part of the lines defined by $\epsilon=2 \eta^{2}-2 \eta+1$ and $\epsilon=2 \zeta^{2}-2 \zeta+1$. One redefines,

$$
\begin{aligned}
\hat{G}_{I}(\eta) & =G_{I}\left(\eta, 2 \eta^{2}-2 \eta+1\right) \quad \text { and } \\
\hat{G}_{I I}(\zeta) & =G_{I I}\left(\zeta, 2 \zeta^{2}-2 \zeta+1\right)
\end{aligned}
$$

with the examination of $\hat{G}_{I}(\eta)$ and $\hat{G}_{I I}(\zeta)$ convexities $\forall \eta \in[0,1]$ and $\eta \in[0,1]$. Noticing here that

$$
\begin{aligned}
& \operatorname{sgn}\left[\hat{G}_{I}^{\prime \prime}(\eta)\right]=\operatorname{sgn}\left[4 \lambda_{1}^{2} \bar{\lambda}_{9}\left(2 \bar{\lambda}_{8}+\bar{\lambda}_{9}\right)\right]=\operatorname{sgn}\left[\bar{\lambda}_{9}\right] \\
& \operatorname{sgn}\left[\hat{G}_{I I}^{\prime \prime}(\zeta)\right]=\operatorname{sgn}\left[4 \lambda_{2}^{2} \bar{\lambda}_{9}\left(2 \bar{\lambda}_{8}+\bar{\lambda}_{9}\right)\right]=\operatorname{sgn}\left[\bar{\lambda}_{9}\right]
\end{aligned}
$$

where (D20) is taken into account.

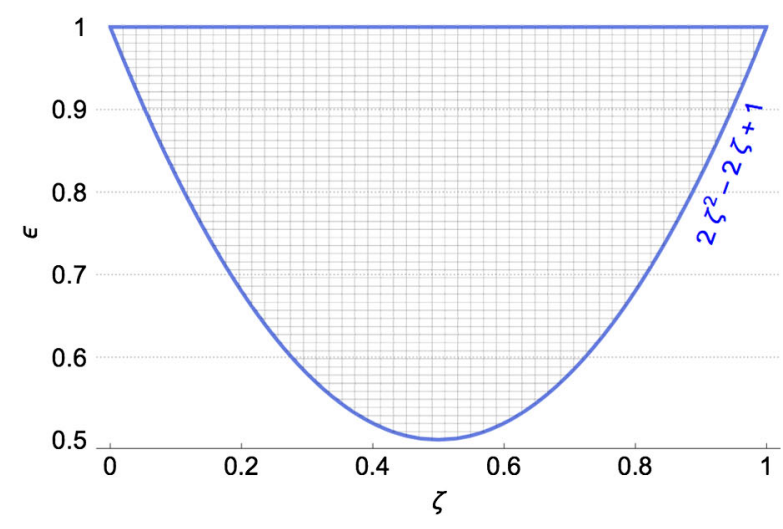

FIG. 12. Dependence of parameters: $(\eta, \epsilon)$ (left) and $(\zeta, \epsilon)$ (right) showing the allowed shaded regions. 
Therefore, one can always find a values $\eta_{0}$ (resp. $\zeta_{0}$ ) for which $\hat{G}_{I}^{\prime}\left(\eta_{0}\right)=0$ (resp. $\hat{G}_{I I}^{\prime}\left(\zeta_{0}\right)=0$ ), so that it may be a minimum only when $\hat{G}_{I}^{\prime \prime}(0)>0 \wedge \hat{G}_{I}^{\prime \prime}(1)<0$ (resp. $\left.\hat{G}_{I I}^{\prime \prime}(0)>0 \wedge \hat{G}_{I I}^{\prime \prime}(1)<0\right)$. This will be true if and only if $\bar{\lambda}_{9} \sqrt{2 \lambda_{1}} \geq\left|\lambda_{8}\right| \sqrt{\bar{\lambda}_{8}+\bar{\lambda}_{9}}$ (resp. $\left.\bar{\lambda}_{9} \sqrt{2 \lambda_{2}} \geq\left|\lambda_{9}\right| \sqrt{\bar{\lambda}_{8}+\bar{\lambda}_{9}}\right)$. In this case,

$$
\hat{G}_{I}\left(\eta_{0}\right)=\lambda_{6}+\frac{1}{2} \lambda_{8}+\frac{1}{2} \sqrt{\left(4 \lambda_{1} \bar{\lambda}_{9}-\lambda_{8}^{2}\right)\left(1+2 \frac{\bar{\lambda}_{8}}{\bar{\lambda}_{9}}\right)}
$$

$\hat{G}_{I I}\left(\zeta_{0}\right)=\lambda_{7}+\frac{1}{2} \lambda_{9}+\frac{1}{2} \sqrt{\left(4 \lambda_{2} \bar{\lambda}_{9}-\lambda_{9}^{2}\right)\left(1+2 \frac{\bar{\lambda}_{8}}{\bar{\lambda}_{9}}\right)}$

We note also that the minimums may situate at $\eta=0 \vee \eta=1$ (resp. $\zeta=0 \vee \zeta=1$ ) from which we get the constraints that both $\hat{G}_{I}(0)=\lambda_{6}+\sqrt{2 \lambda_{1}\left(\bar{\lambda}_{8}+\bar{\lambda}_{9}\right)}$ and $\hat{G}_{I}(1)=$ $\lambda_{6}+\lambda_{8}+\sqrt{2 \lambda_{1}\left(\bar{\lambda}_{8}+\bar{\lambda}_{9}\right)}$ should be positive quantities (resp. $\quad \hat{G}_{I I}(0)=\lambda_{7}+\sqrt{2 \lambda_{2}\left(\bar{\lambda}_{8}+\bar{\lambda}_{9}\right)}$ and $\quad \hat{G}_{I I}(1)=$ $\left.\lambda_{7}+\lambda_{9}+\sqrt{2 \lambda_{2}\left(\bar{\lambda}_{8}+\bar{\lambda}_{9}\right)}\right)$.

As for the Eq. (D28) its implies,

$$
\begin{aligned}
0 & <g(\xi, z)\left(\bar{\lambda}_{8}+\epsilon \bar{\lambda}_{9}\right)-2\left(\lambda_{6}+\eta \lambda_{8}\right)\left(\lambda_{7}+\zeta \lambda_{9}\right)+2 \sqrt{\left(2 \lambda_{1}\left(\bar{\lambda}_{8}+\epsilon \bar{\lambda}_{9}\right)-\left(\lambda_{6}+\eta \lambda_{8}\right)^{2}\right)\left(2 \lambda_{2}\left(\bar{\lambda}_{8}+\epsilon \bar{\lambda}_{9}\right)-\left(\lambda_{7}+\zeta \lambda_{9}\right)^{2}\right)} \\
& \equiv G_{I I I}(\eta, \zeta, \epsilon)
\end{aligned}
$$

where $g(\xi, z)=4\left(\lambda_{3}+\lambda_{4} \xi^{2}+\lambda_{5} \xi^{2} z\right)$.

The minimum of $G_{I I I}(\eta, \zeta, \epsilon)$, as previously, occurs at the border of the dashed line in Fig. 13, so that we can replace either $\epsilon \rightarrow 2 \eta^{2}-2 \eta+1$ or $\epsilon \rightarrow 2 \zeta^{2}-2 \zeta+1$ in which function of two independent variables could be simplified. If the first proposal was retained, the function $G_{I I I}(\eta, \zeta, \epsilon)$ becomes

$$
\begin{aligned}
\hat{G}_{I I I}(\eta, \zeta) \equiv & g(\xi, z)\left(\bar{\lambda}_{8}+\bar{\lambda}_{9}\left(2 \eta^{2}-2 \eta+1\right)\right)-2\left(\lambda_{6}+\eta \lambda_{8}\right)\left(\lambda_{7}+\zeta \lambda_{9}\right) \\
& +2 \sqrt{\left(2 \lambda_{1}\left(\bar{\lambda}_{8}+\bar{\lambda}_{9}\left(2 \eta^{2}-2 \eta+1\right)\right)-\left(\lambda_{6}+\eta \lambda_{8}\right)^{2}\right)\left(2 \lambda_{2}\left(\bar{\lambda}_{8}+\bar{\lambda}_{9}\left(2 \eta^{2}-2 \eta+1\right)\right)-\left(\lambda_{7}+\zeta \lambda_{9}\right)^{2}\right)}
\end{aligned}
$$

In order to find out if such function is convex or not, differentiation is needed and one can easily check that:

$$
\Delta \hat{G}_{I I I}(\eta, \zeta) \equiv\left(\begin{array}{c}
\frac{\partial \hat{G}_{I I I}}{\partial \eta} \\
\frac{\partial \hat{G}_{I I I}}{\partial \zeta}
\end{array}\right) \equiv\left(\begin{array}{l}
F_{1}\left(\lambda_{i}, \eta, \zeta\right) \\
F_{2}\left(\lambda_{i}, \eta, \zeta\right)
\end{array}\right)
$$

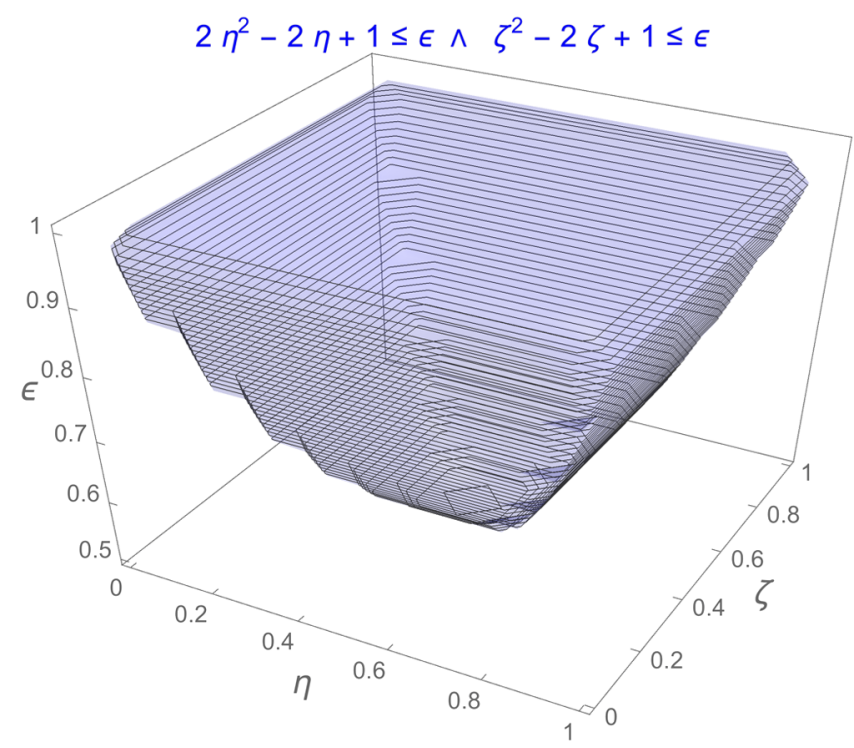

FIG. 13. The simultaneous variation of $\epsilon$ as a function of $\eta$ and $\zeta$ showing the allowed dashed-line region. where the functions $F_{1}, F_{2}$ have a complex forms in $\lambda_{i}, \eta, \zeta$.

Solving the above two stationary points give rise to one solution given by,

$$
\left(\eta_{0}, \zeta_{0}\right)_{1},\left(\eta_{0}, \zeta_{0}\right)_{2},\left(\eta_{0}, \zeta_{0}\right)_{3} \text { and }\left(\eta_{0}, \zeta_{0}\right)_{4}
$$

Therefore, we examine the Hessian of $\hat{G}_{I I I}$ as follows,

$$
H=\left(\begin{array}{ll}
\frac{\partial^{2} \hat{G}_{I I I}}{\partial^{2} \eta} & \frac{\partial^{2} \hat{G}_{I I I}}{\partial \eta \partial \zeta} \\
\frac{\partial^{2} \hat{G}_{I I I}}{\partial \zeta \partial \eta} & \frac{\partial^{2} \hat{G}_{I I I}}{\partial^{2} \zeta}
\end{array}\right)=\left(\begin{array}{ll}
H_{11} & H_{12} \\
H_{21} & H_{22}
\end{array}\right)
$$

where the matrix elements $H_{i j}=H_{i j}\left(\lambda_{i}, \eta, \zeta\right)$.

At the points given in (D41), the eigenvalues of $\mathrm{H}$ can be expressed in terms of $\lambda^{\prime} s$ by solving the following equation:

$$
\left|H-\Lambda \mathbb{1}_{2}\right|=\left|\begin{array}{cc}
H_{11}-\Lambda & H_{12} \\
H_{21} & H_{22}-\Lambda
\end{array}\right|
$$

Hence, at this stage, and for $\hat{G}_{I I I}$ to be convex, the eigenvalues $\Lambda_{1}$ and $\Lambda_{2}$ for each point should be positive quantities. Within sight of their long and complicated expressions, we will not show them here, though they would be taken into account in our calculations. Furthermore, one must also make sure that for each point, both $0 \leq \eta_{0}, \zeta_{0} \leq 1$ for which we request that, 


$$
\hat{G}_{I I I}\left(\eta_{0}, \zeta_{0}\right)>0
$$

$\forall \xi \in[0,1]$ and $z \in[-1,1]$.

The remaining possibility is that the minimum of $\hat{G}_{I I I}$ is at $(\eta, \zeta)=(0,0),(0,1),(1,0)$, or $(1,1)$, from which we get the constraints that

$$
\begin{aligned}
& 0<\left(\bar{\lambda}_{8}+\bar{\lambda}_{9}\right) g(\xi, z)-2\left(\lambda_{6}+\lambda_{8}\right)\left(\lambda_{7}+\lambda_{9}\right)+2 \sqrt{\left(2 \lambda_{1}\left(\bar{\lambda}_{8}+\bar{\lambda}_{9}\right)-\left(\lambda_{6}+\lambda_{8}\right)^{2}\right)\left(2 \lambda_{2}\left(\bar{\lambda}_{8}+\bar{\lambda}_{9}\right)-\left(\lambda_{7}+\lambda_{9}\right)^{2}\right)} \\
& 0<\left(\bar{\lambda}_{8}+\bar{\lambda}_{9}\right) g(\xi, z)-2\left(\lambda_{6}+\lambda_{8}\right) \lambda_{7}+2 \sqrt{\left(2 \lambda_{1}\left(\bar{\lambda}_{8}+\bar{\lambda}_{9}\right)-\left(\lambda_{6}+\lambda_{8}\right)^{2}\right)\left(2 \lambda_{2}\left(\bar{\lambda}_{8}+\bar{\lambda}_{9}\right)-\left(\lambda_{7}+\lambda_{9}\right)^{2}\right)} \\
& 0<\left(\bar{\lambda}_{8}+\bar{\lambda}_{9}\right) g(\xi, z)-2 \lambda_{6} \lambda_{7}+2 \sqrt{\left(2 \lambda_{1}\left(\bar{\lambda}_{8}+\bar{\lambda}_{9}\right)-\lambda_{6}^{2}\right)\left(2 \lambda_{2}\left(\bar{\lambda}_{8}+\bar{\lambda}_{9}\right)-\lambda_{7}^{2}\right)} \\
& 0<\left(\bar{\lambda}_{8}+\bar{\lambda}_{9}\right) g(\xi, z)-2 \lambda_{6}\left(\lambda_{7}+\lambda_{9}\right)+2 \sqrt{\left(2 \lambda_{1}\left(\bar{\lambda}_{8}+\bar{\lambda}_{9}\right)-\lambda_{6}^{2}\right)\left(2 \lambda_{2}\left(\bar{\lambda}_{8}+\bar{\lambda}_{9}\right)-\lambda_{7}^{2}\right) .}
\end{aligned}
$$

[1] G. Aad et al. (ATLAS Collaboration), Phys. Lett. B 716, 1 (2012).

[2] S. Chatrchyan et al. (CMS Collaboration), Phys. Lett. B 716, 30 (2012).

[3] C. Patrignani et al. (Particle Data Group), Chin. Phys. C 40, 100001 (2016).

[4] S. Weinberg, Phys. Rev. Lett. 43, 1566 (1979).

[5] P. Minkowski, Phys. Lett. 67B, 421 (1977).

[6] M. Gell-Mann, P. Ramond, and R. Slansky, Conf. Proc. C 790927, 315 (1979).

[7] T. Yanagida, Conf. Proc. C 7902131, 95 (1979).

[8] R. N. Mohapatra and G. Senjanovic, Phys. Rev. Lett. 44, 912 (1980).

[9] J. Schechter and J. W. F. Valle, Phys. Rev. D 22, 2227 (1980).

[10] R. N. Mohapatra and G. Senjanovic, Phys. Rev. D 23, 165 (1981).

[11] G. Lazarides, Q. Shafi, and C. Wetterich, Nucl. Phys. B181, 287 (1981).

[12] C. Wetterich, Nucl. Phys. B187, 343 (1981).

[13] J. Schechter and J. W. F. Valle, Phys. Rev. D 25, 774 (1982).

[14] R. Foot, H. Lew, X. G. He, and G. C. Joshi, Z. Phys. C 44, 441 (1989).

[15] A. Arhrib, R. Benbrik, M. Chabab, G. Moultaka, M. C. Peyranere, L. Rahili, and J. Ramadan, Phys. Rev. D 84, 095005 (2011).

[16] A. Arhrib, R. Benbrik, M. Chabab, G. Moultaka, and L. Rahili, J. High Energy Phys. 04 (2012) 136.

[17] M. Chabab, M. C. Peyranere, and L. Rahili, Phys. Rev. D 90, 035026 (2014).

[18] M. Chabab, M. C. Peyranere, and L. Rahili, Phys. Rev. D 93, 115021 (2016).

[19] A. Arhrib, R. Benbrik, G. Moultaka, and L. Rahili, arXiv: 1411.5645 .

[20] D. A. Camargo, A. G. Dias, T. B. de Melo, and F. S. Queiroz, J. High Energy Phys. 04 (2019) 129.
[21] C. H. Chen and T. Nomura, Phys. Rev. D 90, 075008 (2014).

[22] A. Arhrib, arXiv:hep-ph/0012353.

[23] A. G. Akeroyd, A. Arhrib, and E. M. Naimi, Phys. Lett. B 490, 119 (2000).

[24] D. A. Ross and M. Veltman, Nucl. Phys. B95, 135 (1975); M. Veltman, Nucl. Phys. B123, 89 (1977).

[25] C. Patrignani et al. (Particle Data Group), Chin. Phys. C 40, 100001 (2016).

[26] A. Goudelis, B. Herrmann, and O. Stal, J. High Energy Phys. 09 (2013) 106.

[27] L. Lavoura and L. F. Li, Phys. Rev. D 49, 1409 (1994).

[28] P. Bechtle, O. Brein, S. Heinemeyer, G. Weiglein, and K. E. Williams, Comput. Phys. Commun. 182, 2605 (2011); 181, 138 (2010).

[29] G. Aad et al. (ATLAS and CMS Collaborations), Phys. Rev. Lett. 114, 191803 (2015).

[30] P. Bechtle, S. Heinemeyer, O. Stal, T. Stefaniak, and G. Weiglein, Eur. Phys. J. C 74, 2711 (2014).

[31] C. S. Lim and T. Inami, Prog. Theor. Phys. 67, 1569 (1982); A. Pich, A. Santamaria, and J. Bernabeu, Phys. Lett. 148B, 229 (1984); M. L. Swartz, Phys. Rev. D 40, 1521 (1989); M. Raidal and A. Santamaria, Phys. Lett. B 421, 250 (1998).

[32] E. J. Chun, K. Y. Lee, and S. C. Park, Phys. Lett. B 566, 142 (2003).

[33] M. Kakizaki, Y. Ogura, and F. Shima, Phys. Lett. B 566, 210 (2003).

[34] A. G. Akeroyd, M. Aoki, and H. Sugiyama, Phys. Rev. D 79, 113010 (2009).

[35] E. Ma, M. Raidal, and U. Sarkar, Phys. Rev. Lett. 85, 3769 (2000); E. Ma, M. Raidal, and U. Sarkar, Nucl. Phys. B615, 313 (2001).

[36] Y. Kuno and Y. Okada, Rev. Mod. Phys. 73, 151 (2001).

[37] L. Lavoura, Eur. Phys. J. C 29, 191 (2003).

[38] U. Bellgardt et al. (SINDRUM Collaboration), Nucl. Phys. B299, 1 (1988). 
[39] A. M. Baldini et al. (MEG Collaboration), Eur. Phys. J. C 76, 434 (2016).

[40] G. Aad et al. (ATLAS Collaboration), J. High Energy Phys. 01 (2016) 032.

[41] G. Aad et al. (ATLAS Collaboration), Eur. Phys. J. C 76, 45 (2016).

[42] V. Khachatryan et al. (CMS Collaboration), J. High Energy Phys. 10 (2015) 144.

[43] Search for a high-mass Higgs boson decaying to a pair of W bosons in pp collisions at $\sqrt{(} s)=13 \mathrm{TeV}$ with the ATLAS detector, CERN Technical Report No. ATLAS-CONF2016-021, 2016.

[44] Search for ZZ resonances in the qq final state in pp collisions at $\sqrt{s}=13 \mathrm{TeV}$ with the ATLAS detector, CERN Technical Report No. ATLAS-CONF-2016-016, 2016.

[45] Search for high-mass resonances decaying into a $\mathrm{Z}$ boson pair in the ll $\nu \nu$ final state in pp collisions at $\sqrt{s}=13 \mathrm{TeV}$ with the ATLAS detector, Technical Report No. ATLASCONF-2016-012, CERN, Geneva, 2016.

[46] Search for new phenomena in the $\mathrm{Z}(\rightarrow 11)+E_{T}^{\text {miss }}$ final state at $\sqrt{s}=13 \mathrm{TeV}$ with the ATLAS detector, CERN Technical Report No. ATLAS-CONF-2016-056, 2016.

[47] Search for high mass Higgs to WW with fully leptonic decays using 2015 data, CERN Technical Report No. CMSPAS-HIG-16-023, 2016.

[48] R. V. Harlander, S. Liebler, and H. Mantler, Comput. Phys. Commun. 184, 1605 (2013).

[49] R. V. Harlander, S. Liebler, and H. Mantler, Comput. Phys. Commun. 212, 239 (2017).
[50] Searches for heavy ZZ and ZW resonances in the llqq and vvqq final states in pp collisions at $\sqrt{(} s)=13 \mathrm{TeV}$ with the ATLAS detector, CERN Technical Report No. ATLASCONF-2016-082, 2016.

[51] Study of the Higgs boson properties and search for highmass scalar resonances in the $H \rightarrow Z Z^{*} \rightarrow 4 l$ decay channel at $\sqrt{(} s)=13 \mathrm{TeV}$ with the ATLAS detector, CERN Technical Report No. ATLAS-CONF-2016-079, 2016.

[52] Search for diboson resonance production in the $l v q q$ final state using pp collisions at $\sqrt{(} s)=13$ with the ATLAS detector at the LHC, CERN Technical Report No. ATLASCONF-2016-062, 2016.

[53] Search for a high-mass Higgs boson decaying to a pair of W bosons in pp collisions at $\sqrt{(} s)=13 \mathrm{TeV}$ with the ATLAS detector, CERN Technical Report No. ATLAS-CONF2016-074, 2016.

[54] Search for additional heavy neutral Higgs and gauge bosons in the ditau final state produced in $36.1 \mathrm{fb}^{-1}$ of pp collisions at $\sqrt{s}=13 \mathrm{TeV}$ with the ATLAS detector, CERN Technical Report No. ATLAS-CONF-2017-050, 2017.

[55] Search for a neutral MSSM Higgs boson decaying into $\tau \tau$ with $12.9 \mathrm{fb}^{-1}$ of data at $\sqrt{s}=13 \mathrm{TeV}$, CERN Technical Report No. CMS-PAS-HIG-16-037, 2016

[56] Search for scalar diphoton resonances with $15.4 \mathrm{fb}^{-1}$ of data collected at $\sqrt{s}=13 \mathrm{TeV}$ in 2015 and 2016 with the ATLAS detector, CERN Technical Report No. ATLASCONF-2016-059, 2016.

[57] C. Bonilla, R. M. Fonseca, and J. W. F. Valle, Phys. Rev. D 92, 075028 (2015). 